\title{
Long-Lived Interacting Phases of Matter Protected by Multiple Time-Translation Symmetries in Quasiperiodically Driven Systems
}

\author{
Dominic V. Else, ${ }^{1, *}$ Wen Wei Ho๑ ${ }^{2, \dagger}$ and Philipp T. Dumitrescu $\oplus^{3, \$}$ \\ ${ }^{1}$ Department of Physics, Massachusetts Institute of Technology, Cambridge, Massachusetts 02139, USA \\ ${ }^{2}$ Department of Physics, Harvard University, Cambridge, Massachusetts 02138, USA \\ ${ }^{3}$ Center for Computational Quantum Physics, Flatiron Institute, \\ 162 Fifth Avenue, New York, New York 10010, USA
}

(Received 15 October 2019; accepted 19 March 2020; published 11 May 2020)

\begin{abstract}
We show how a large family of interacting nonequilibrium phases of matter can arise from the presence of multiple time-translation symmetries, which occur by quasiperiodically driving an isolated, quantum many-body system with two or more incommensurate frequencies. These phases are fundamentally different from those realizable in time-independent or periodically driven (Floquet) settings. Focusing on high-frequency drives with smooth time dependence, we rigorously establish general conditions for which these phases are stable in a parametrically long-lived "preheating" regime. We develop a formalism to analyze the effect of the multiple time-translation symmetries on the dynamics of the system, which we use to classify and construct explicit examples of the emergent phases. In particular, we discuss time quasicrystals which spontaneously break the time-translation symmetries, as well as time-translation symmetry-protected topological phases.
\end{abstract}

DOI: 10.1103/PhysRevX.10.021032

\section{INTRODUCTION}

Many-body quantum systems give rise to a vast array of interesting phases of matter. The last decade has seen a dramatic expansion and refinement in our understanding of the landscape of such phases [1-4]. Recently, it was shown how time-translational symmetry (TTS) can give rise to and protect intrinsically out-of-equilibrium phases of matter in isolated quantum systems [5-8]. Arguably, the simplest example is the discrete time crystal [7,9-14], characterized by the spontaneous breaking of the discrete TTS of a periodic (Floquet) drive. This is manifested in physical observables exhibiting robust, long-lived oscillations at an integer multiple of the base driving period. Experimental signatures of this behavior have been reported in various platforms [15-17]. Going beyond discrete time crystals, a large number of other Floquet phases in which TTS plays an essential role, including

\footnotetext{
d_else@mit.edu

wenweiho@fas.harvard.edu

*pdumitrescu@flatironinstitute.org
}

Published by the American Physical Society under the terms of the Creative Commons Attribution 4.0 International license. Further distribution of this work must maintain attribution to the author(s) and the published article's title, journal citation, and DOI.
Subject Areas: Atomic and Molecular Physics, Condensed Matter Physics

topological phases beyond the equilibrium classification, have also been discussed.

The richness of Floquet phases naturally raises the following questions: Are there fundamentally different nonequilibrium interacting phases beyond Floquet, which are not dynamically engineered analogs of static phases? What is the role of TTS in characterizing these phases?

Apart from theoretical interest, these questions are placed upon us by the dramatic experimental advances in controlling and manipulating isolated quantum manybody systems, such as cold atoms [18], trapped ions [19$21]$, nitrogen-vacancy centers [22,23], and superconducting qubits $[24,25]$. These systems provide a natural platform to realize dynamical protocols, allowing us to systematically study physics in out-of-equilibrium settings, including thermalization and equilibration. Classifying nonequilibrium phases tells us exactly what long-time, dynamical collective behaviors are possible and the universal features defining them.

However, driven interacting systems are generically expected to heat up to a featureless infinite-temperature state due to a lack of energy conservation [26-30]. Thus, to meaningfully define phases of matter in such settings, systems must be protected against heating, at least for some long timescale. For Floquet systems, this challenge can be overcome by applying high-frequency drives leading to exponentially long-lived prethermal regimes [31-36] or by applying strong disorder, leading to many-body 
localization (MBL) [30,37,38]. Generalizing these ideas to more generic driving scenarios remains an important open question.

In this paper, we consider interacting quantum manybody systems subject to a quasiperiodic drive that consists of several incommensurate frequencies and is smooth in time. We rigorously show that under such driving scenarios, the system is protected at high driving frequencies from heating for a parametrically long time, giving rise to a so-called "preheating" regime. The heating time scales as a stretched exponential of the ratio of the drive frequency to local coupling strengths. We demonstrate, through a recursive construction, that there is an effective static Hamiltonian governing time evolution in the preheating regime, which generalizes the Floquet analysis of Refs. [12,35] to a large class of new dynamical systems.

The presence of a preheating regime in quasiperiodically driven systems opens up an avenue to realize novel, longlived, nonequilibrium phases of matter. We provide a set of general driving conditions to realize these phases and discuss how they are distinguished by a notion of multiple time-translation symmetries (TTSs) of the drives; thus, they are fundamentally different from those in static or Floquet settings. In particular, we classify two exemplars of such phases: the discrete time quasicrystal (DTQC), which spontaneously breaks the multiple TTSs, and quasiperiodic symmetry-protected topological (SPT) and symmetryenriched topological (SET) phases, which are protected by them. Our results showcase the richness of the landscape of quasiperiodically driven phases and open up new directions in the rapidly developing field of nonequilibrium quantum matter.

Before we proceed, let us note that the study of the dynamics of quantum systems under quasiperiodic driving has a venerable history, encompassing diverse applications from experiments in chemistry and physics, to the basic structure of first-order differential equations [39-58]. Interesting dynamical behavior related to topology in fewbody or noninteracting scenarios have also been reported [59-63]. Many-body quasiperiodically driven quantum systems with interactions have received increasing attention recently [64-66]. Our approach is distinguished from previous studies in that we explicitly establish the stability of the nonequilibrium phases we discuss, by rigorously providing a bound on their lifetimes. We additionally demonstrate the robustness of their universal properties against small changes in the driving protocol, which justifies their characterization as "phases of matter."

The outline of this paper is as follows. In Sec. II, we summarize our main results on establishing a long preheating regime in quasiperiodically driven systems, in which one can discuss phases of matter. In Sec. III, we introduce the notion of a "frame-twisted high-frequency limit," which will allow us to find new phases of matter with no static or periodically driven analogs. We show how TTSs act in this regime and make this precise by defining "twisted timetranslation symmetries." In Sec. IV, we discuss spontaneous symmetry breaking for the multiple TTSs, which lead to discrete time quasicrystal phases. In Sec. V, we define and classify topological phases protected by the multiple TTSs. In Sec. VI, we return to the stability of the prethermal regime and show how the scaling of the heating time with frequency can be intuitively understood in terms of simple linear response arguments. In Sec. VII, we state and sketch the proof of our rigorous results on the heating bounds and on the description of the dynamics; technical details are relegated to Appendix H. Finally, in Sec. VIII, we discuss various extensions and future directions, and we conclude in Sec. IX.

Note that the term "time quasicrystal" (TQC) has been used for systems that show a quasiperiodic response, arising from either a quasiperiodic [64] or a periodic drive $[67,68]$. In this paper, we restrict our use of the term time quasicrystal to the first sense, where a quasiperiodic drive gives rise to a response with a different quasiperiodic pattern (see Sec. IV).

\section{OVERVIEW: MAIN IDEAS AND KEY RESULTS}

\section{A. Multiple time-translational symmetries in quasiperiodically driven systems}

In this paper, we show the existence of long-lived nonequilibrium phases of matter protected by multiple TTSs in quasiperiodically driven systems, which are defined as follows. Consider an at-least piecewise continuous Hamiltonian $H(\boldsymbol{\theta})$ parametrized by the $m$-dimensional "standard" torus $\mathbb{T}^{m} \ni \boldsymbol{\theta}=\left(\theta_{1}, \ldots, \theta_{m}\right)$, which is $2 \pi$ periodic in each angle $\theta_{i}$. Additionally, let us pick a vector of rationally independent frequencies $\boldsymbol{\omega}=\left(\omega_{1}, \ldots, \omega_{m}\right)$ so that $\boldsymbol{n} \cdot \boldsymbol{\omega} \neq 0$ for any nonzero integer vector $\boldsymbol{n} \in \mathbb{Z}^{m}$. When $m=2$, it suffices to choose the ratio $\omega_{2} / \omega_{1}$ to be an irrational number, such as the golden ratio $\omega_{2} / \omega_{1}=\varphi=(1+\sqrt{5}) / 2$. The dynamics of the system under the time-dependent Hamiltonian

$$
H(t)=H\left(\boldsymbol{\omega} t+\boldsymbol{\theta}_{0}\right)
$$

is time quasiperiodic for $m \geq 2$ and constitutes a quasiperiodic drive. Here, $t$ is the physical "single" time, and $\boldsymbol{\theta}_{0} \in \mathbb{T}^{m}$ are some fixed initial angles (see Fig. 1). The class of drives described by Eq. (1) encompasses Floquet driving as the case $m=1$, which will enable us to directly compare our analysis to previous work. Note that throughout the paper, we use the same symbol-e.g., $H$ above - to refer to the same physical quantity viewed either as a function on the torus, $H(\boldsymbol{\theta})$, or as a function of single time, $H(t)$. As in Eq. (1), the single time function is obtained by evaluating the torus function along the particular trajectory $\boldsymbol{\theta}(t)=$ $\boldsymbol{\omega} t+\boldsymbol{\theta}_{0}$. While these are technically different mathematical 
(a)

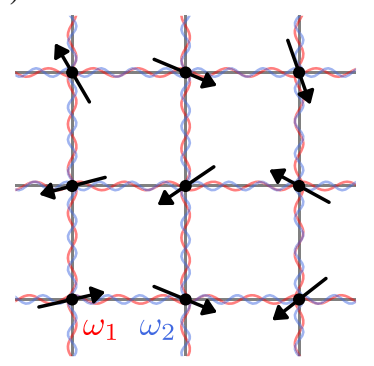

(b) $\theta_{2}$

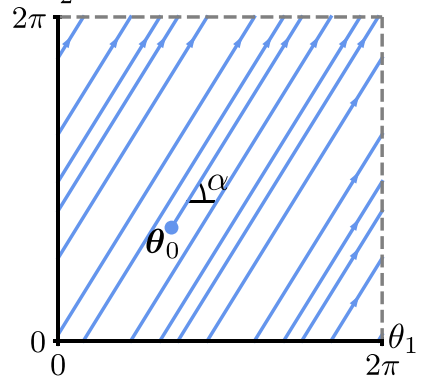

FIG. 1. (a) Cartoon of an ensemble of spins that is quasiperiodically driven. (b) A time-quasiperiodic Hamiltonian $H(t)$ arises from evaluating a Hamiltonian $H(\boldsymbol{\theta})$ defined on the $m$-dimensional torus $\mathbb{T}^{m} \ni \boldsymbol{\theta}$ on the trajectory $\boldsymbol{\theta}(t)=\boldsymbol{\omega} t+\boldsymbol{\theta}_{0}$ (blue arrows). As the frequency vector $\omega$ is a set of rationally independent frequencies, the trajectory never returns to itself but covers the torus uniformly. Shown here is the case $m=2$, with $\tan \alpha=\omega_{2} / \omega_{1}$ irrational.

functions, they can be distinguished by their scalar $t$ or vector $\boldsymbol{\theta}$ arguments.

At first glance, it is puzzling how one could obtain phases protected by TTSs in quasiperiodically driven systems. After all, the incommensurate nature of the driving frequencies implies that the Hamiltonian Eq. (1) does not even have a single time-translational symmetry [there is no period $T \neq 0$ for which $H(t)=H(t+T)$ for all $t$, let alone multiple TTSs. However, $H(t)$ derives from an underlying $H(\boldsymbol{\theta})$ on $\mathbb{T}^{m}$ through Eq. (1), which is symmetric under translations $\boldsymbol{\theta} \rightarrow \boldsymbol{\theta}+\boldsymbol{\tau}$. Here, the translation vectors belong to a lattice $\tau \in \mathcal{L}=2 \pi \mathbb{Z}^{m}$ generated by $m$ independent symmetries. It is thus conceivable that these symmetries have meaningful and nontrivial implications for the single-time system. Since for $m=1$ these symmetries reduce to the time-translation symmetry of a Floquet system, we still refer to these symmetries as "time-translation symmetries" for quasiperiodically driven systems $(m \geq 2)$.

In what follows, we make the above statements precise by interpreting the consequences of the multiple TTSs in a certain class of physical systems. Specifically, we consider quantum many-body systems defined on lattices in arbitrary spatial dimensions with bounded local Hilbert spaces (i.e., spins or fermions), which respect a sense of localitythe interaction strength decays sufficiently fast with distance. In particular, we allow for a Hamiltonian having interactions with amplitude that are at least exponentially decaying with distance (termed "quasilocal"); see Sec. VII. We show the following:

(1) For such strongly interacting many-body systems under some non-fine-tuned quasiperiodic driving conditions, the multiple time-translation symmetries of $H(\boldsymbol{\theta})$ give rise to an actual symmetry of the effective time-independent Hamiltonian that describes the dynamics in a long-lived preheating

regime. This result enables the existence of novel nonequilibrium phases of matter protected by these symmetries.

(2) The classification of quasiperiodically driven manybody phases of matter-both spontaneous symmetry breaking and topological phases-is the same as the classification of equilibrium phases with a symmetry group extended by $\mathbb{Z}^{\times m}$. This is a direct generalization of the $m=1$ Floquet results of Ref. [69].

\section{B. Long lifetimes in quasiperiodically driven systems}

Owing to the lack of energy conservation, a generic ergodic interacting driven system is expected to heat to a featureless infinite-temperature state, where symmetries act trivially and a discussion of phases of matter is moot. Therefore, before we can even discuss new phases realizable with multiple TTSs, we must establish that there exist suitable quasiperiodic driving conditions where such deleterious heating is controlled, at least for some parametrically long time.

In Floquet systems $(m=1)$, the conditions needed to achieve this are relatively mild. Energy absorption or emission between the system and the drive can only take place in integer multiples of the driving frequency, i.e., $\Delta E=n \omega$ for integer $n$. By suppressing resonances between energy eigenstates connected by such discrete energy levels, heating can be slow. Both Floquet-MBL and Floquet prethermalization involve suppressing heating processes in such a way, though through different physical mechanisms. The former uses strong disorder to directly curtail the probability of local resonances $[30,37,38]$. The latter entails driving at such high frequencies compared to local energy scales $J \ll \omega$ that the system can only absorb the large drive quanta $n \omega$ by performing a multiple-spin rearrangement. This rate is heavily suppressed, giving rise to a long heating timescale $t_{*} \sim e^{\text {const } \omega / J}$ [31]. Other scenarios where heating is slow, which do not result from disorder or high-frequency driving, have also been considered; see Refs. [70-72].

In quasiperiodically driven systems $(m \geq 2)$, by contrast, energy absorption or emission occurs in units of $\Delta E_{n}=$ $\boldsymbol{\omega} \cdot \boldsymbol{n}$ for any integer vector $\boldsymbol{n} \in \mathbb{Z}^{m}$. As the frequencies $\boldsymbol{\omega}=\left(\omega_{1}, \ldots, \omega_{m}\right)$ are rationally independent, the set of all possible such quanta $\Delta E_{n}$ is dense on the real line. Superficially, it seems impossible to avoid immediate heating.

However, a more careful consideration shows that this is not necessarily an insurmountable problem. Consider, as an example, a static Hamiltonian $H_{0}$ weakly driven by a quasiperiodic perturbation $V(t)=V\left(\boldsymbol{\omega} t+\boldsymbol{\theta}_{0}\right)$. Expanding $V(\boldsymbol{\theta})$ in a Fourier series

$$
V(\boldsymbol{\theta})=\sum_{n \in \mathbb{Z}^{m}} V_{n} e^{i n \cdot \boldsymbol{\theta}},
$$


one sees that $V_{n}$ induces transitions between energy levels of $H_{0}$ separated by energies $\Delta E_{n}$ in linear response theory. The set of all $\Delta E_{n}$ with $|\boldsymbol{n}|$ below some cutoff becomes ever more closely spaced as the cutoff increases. Nonetheless, as long as the amplitude $\left\|V_{\boldsymbol{n}}\right\|$ decays fast enough with $|\boldsymbol{n}|$ as compared to this spacing, resonances will not proliferate at large $|\boldsymbol{n}|$. This observation suggests a restriction of $V(\boldsymbol{\theta})$ to be "sufficiently" smooth on the torus, which in turn translates to smooth drives in time. Resonances arising from small $|\boldsymbol{n}|$ processes also need to be suppressed-but this suppression should be achievable with the same mechanisms as in the Floquet case (strong disorder or high-frequency driving).

One of the key aspects of our present work is to make this plausible stability statement concrete. Specifically, we consider the case corresponding to a quasiperiodically driven many-body Hamiltonian $H\left(\boldsymbol{\omega} t+\boldsymbol{\theta}_{0}\right)$ under two conditions. First, that it is smooth in time, in the sense that the amplitude of the Fourier coefficients $H_{n}$ of the driving Hamiltonian decay as $\left\|H_{\boldsymbol{n}}\right\| \lesssim e^{- \text {const }|\boldsymbol{n}|}$ at large $|\boldsymbol{n}|$. Second, that the driving frequencies $\omega_{i}$ are large compared to any local energy scales $J$ of the Hamiltonian. Under these conditions, we show that the heating time $t_{*}$ is rigorously bounded for any $\epsilon>0$ and for all except a set of measure zero choices of frequency vectors $\omega$ by

$$
t_{*} \geq \frac{C^{\prime}}{J} \exp \left[C\left(\frac{\omega}{J}\right)^{1 /(m+e)}\right] .
$$

Here, $\omega=|\omega|$ is the norm of the driving frequency, $C, C^{\prime}$ are dimensionless numbers depending on the numbertheoretic properties of the irrational ratios $\omega_{i} / \omega_{j}$, and $m$ is the number of incommensurate frequencies. Thus, keeping the ratios $\omega_{i} / \omega_{j}$ fixed, the bound on heating time $t_{*}$ follows a simple stretched exponential in frequency dependence. While this result can be intuitively understood within linear response theory (Sec. VI), we prove the bound in Eq. (3) using a recursive construction beyond linear response (Sec. VII). Note that for nonsmooth drives, where $V_{n}$ decays more slowly than exponentially at large $|\boldsymbol{n}|$, the heating time $t_{*}$ scales with a different functional form. For a power-law decay of $V_{n}$, such as for step drives, we expect that $t_{*}$ scales as a power law in $\omega / J$ (see Sec. VIII C). While Eq. (3) is analogous to the high-frequency heating bound known for Floquet systems, the heating time in those systems is always a simple exponential in $\omega / J$ without restrictions on the drive smoothness.

\section{Description of preheating dynamics}

The existence of a long timescale [Eq. (3)] implies a long-lived preheating regime and opens up the possibility to define phases of matter in this time interval. How can we concretely describe the dynamics, and eventually characterize phases, in the preheating regime?
Let us briefly recall the Floquet scenario $(m=1)$, where, generally, the dynamics in a preheating regime is approximately governed by an effective, static, quasilocal Hamiltonian $D$. More precisely, the time-evolution operator $U(t)=\mathcal{T} \exp \left[-i \int_{0}^{t} H\left(t^{\prime}\right) d t^{\prime}\right]$ can be written as

$$
U(t) \approx P(t) e^{-i D t} P^{\dagger}(0),
$$

where $P(t)=P(t+T)$ is a unitary change of frame that is periodic in time, and the approximate equality reflects an omission of small local terms in the Hamiltonian that do not affect the dynamics up to the heating time $t_{*}$ [32-35]. A quantum state's dynamical evolution can therefore be understood as comprised of two parts: time evolution generated by the static Hamiltonian $D$ and an additional "micromotion" governed by $P(t)$. If we only consider the state of the system at stroboscopic times, i.e., integer multiples of the driving period, then the dynamics is generated by the Floquet operator $U_{F}:=U(T)$. This operator can be written [if we choose $P(0)=P(T)=\mathbb{I}$ ] as $U_{F} \approx e^{-i D T}$, in which case the study of the dynamical evolution of the system up to time $t_{*}$ entails studying the eigenstates of the Hamiltonian $D$.

We strongly emphasize here the importance of $D$ being quasilocal. In fact, the Floquet-Bloch theorem asserts that the decomposition in Eq. (4) exists with an exact equality if $D$ is replaced by the Floquet Hamiltonian $H_{F}$. However, $H_{F}$ will be highly nonlocal in a generic ergodic many-body system - it must, after all, describe the eventual heating to an infinite-temperature state-and therefore is not very insightful to use when studying the preheating regime, as compared to $D$.

One scenario where a long heating time emerges and the approximate decomposition (4) holds is in the limit of highfrequency driving, in which case $t_{*} \gtrsim e^{\text {const } \omega / J}$. The small ratio of the local energy scale to the driving frequency $J / \omega$ naturally enables schemes for an order-by-order expansion of $D$ and $P$. For example, the commonly used "FloquetMagnus" expansion [5,73] with $P(0)=P(T)=\mathbb{I}$ gives, at lowest orders,

$$
\begin{aligned}
& D^{(0)}=\frac{1}{T} \int_{0}^{T} d t^{\prime} H\left(t^{\prime}\right), \\
& D^{(1)}=\frac{1}{2 T} \int_{0}^{T} d t^{\prime} \int_{0}^{t^{\prime}} d t^{\prime \prime}\left[H\left(t^{\prime}\right), H\left(t^{\prime \prime}\right)\right],
\end{aligned}
$$

and is generally an asymptotic series. References [32,33] showed that if truncated at some optimal order, Eq. (4) is satisfied with an error of $\sim 1 / t_{*}=O\left(e^{- \text {const } \omega / J}\right)$. Reference [34] also constructed an effective static Hamiltonian that provably approximates the dynamics until the same $t_{*}$, although it was not directly expressed in terms of the Floquet-Magnus expansion.

We now return to quasiperiodically driven systems $(m \geq 2)$. For the smooth high-frequency drives that we 
consider, we prove that a similar decomposition of the unitary time-evolution operator $U(t)$ as in Eq. (4) exists,

$$
U(t) \approx P\left(\boldsymbol{\omega} t+\boldsymbol{\theta}_{0}\right) e^{-i D t} P^{\dagger}\left(\boldsymbol{\theta}_{0}\right) .
$$

A quantum state's dynamics is again effectively comprised of time evolution by some static Hamiltonian $D$ and a micromotion given by some unitary time-quasiperiodic change of frame $P(t):=P\left(\boldsymbol{\omega} t+\boldsymbol{\theta}_{0}\right)$ with underlying $P(\boldsymbol{\theta})$ smooth on the torus. In Sec. VII, we demonstrate how to construct the effective Hamiltonian $D$ and unitary $P$ through an iterative renormalization procedure of the driving Hamiltonian $H(t)$ that can be understood as a generalization of the methods of Ref. [34], as well as bound the optimal order to which the procedure should be carried out. This gives rise to an optimal $D$ such that the description in Eq. (6) is valid at least for times $t \lesssim t_{*}$, with $t_{*}$ satisfying Eq. (3).

While it seems natural to assume that the decomposition in Eq. (4) carries over from the Floquet case to quasiperiodically driven systems, this is far from obvious. It is rigorously known that the decomposition in Eq. (6) with an exact equality (i.e., Floquet-Bloch theorem) is not guaranteed in general quasiperiodic systems, there being obstructions to defining a generalized Floquet Hamiltonian [41]. To understand why one expects Eq. (6) to hold in the quasiperiodically driven case with conditions given in Sec. II B, observe that the high-frequency assumption suggests that an expansion analogous to the FloquetMagnus expansion in Eq. (5) can be written down. Representing $H(\boldsymbol{\theta})$ as a Fourier series $H(\boldsymbol{\theta})=\sum_{n} H_{n} e^{i \boldsymbol{n} \cdot \boldsymbol{\theta}}$ and assuming the form of Eq. (6) with $P(t)$ quasiperiodic, one can perform a formal expansion in powers of the inverse norm of driving frequencies $1 / \omega$ of the effective Hamiltonian $D=D^{(0)}+D^{(1)}+\cdots$ as well as the unitary $P(\boldsymbol{\theta})=\exp \left(\boldsymbol{\Omega}^{(1)}(\boldsymbol{\theta})+\boldsymbol{\Omega}^{(2)}(\boldsymbol{\theta})+\cdots\right)$, whose leading-order terms read (see Appendix A)

$$
\begin{aligned}
D^{(0)} & =H_{\mathbf{0}}=\int_{\mathbb{T}^{m}} \frac{d^{m} \boldsymbol{\theta}}{(2 \pi)^{m}} H(\boldsymbol{\theta}), \\
D^{(1)} & =\frac{1}{2} \sum_{\boldsymbol{n}} \frac{1}{\boldsymbol{\omega} \cdot \boldsymbol{n}}\left[H_{\boldsymbol{n}}, H_{-\boldsymbol{n}}\right] .
\end{aligned}
$$

Here, $\Omega^{(1)}(\boldsymbol{\theta})=-\sum_{\boldsymbol{n} \neq \boldsymbol{0}} H_{\boldsymbol{n}} e^{i \boldsymbol{n} \cdot \boldsymbol{\theta}} /(\boldsymbol{n} \cdot \boldsymbol{\omega})$. However, while relatively simple to construct, even the low-order terms in Eq. (7) already signal a difficulty that is not present in the Floquet case: The denominator $\boldsymbol{n} \cdot \boldsymbol{\omega}$ can be arbitrarily small, leading to possible divergences and bringing into question the validity of the expansion. This is precisely the manifestation of the denseness of resonances discussed in Sec. II B. As before, we observe that this issue can potentially be circumvented if the size of the Fourier coefficients $H_{n}$ decays sufficiently rapidly with $|\boldsymbol{n}|$, such as in the case of smooth driving, so that the small denominators are suppressed. Note also that while similar to Eq. (5), the expansion of Eq. (7) does not reduce to it upon setting $m=1$, as we explain in Appendix A. This point will be important in the discussion of emergent symmetries.

A central contribution of our paper is to show how imposing the smoothness conditions on the drive indeed leads to a meaningful high-frequency expansion, which can be used to construct an effective static Hamiltonian. The expansion we develop, which is different from that of Eq. (7), is given in Sec. VII.

\section{Equilibration and steady states in the preheating regime}

Let us now describe the kind of dynamics and "steady states" one can expect in the preheating regime, given an effective, static, quasilocal Hamiltonian $D$ via Eq. (6).

If $D$ is generic and nonintegrable, one expects dynamics from a simple initial state $\left|\psi_{0}\right\rangle$ to lead to thermalization with respect to $D$, when viewed in the time-dependent frame defined by $P(t)$. In other words, the system locally approaches an equilibrium distribution $\rho_{\beta} \propto e^{-\beta D}$, where the inverse temperature $\beta$ depends on the energy of the initial state as measured by $D$. Precisely, $\beta$ is obtained from the relation $\operatorname{Tr}\left(D e^{-\beta D}\right) / Z=\left\langle\psi_{0}\left|P\left(\boldsymbol{\theta}_{0}\right) D P^{\dagger}\left(\boldsymbol{\theta}_{0}\right)\right| \psi_{0}\right\rangle$, where $Z=\operatorname{Tr}\left(e^{-\beta D}\right)$. This happens provided local relaxation timescales $t_{r}$ are much less than the heating timescale $t_{*}$, i.e., $t_{r} \sim J^{-1} \ll t_{*}$, which always occurs for highfrequency drives. As the system is expected to eventually thermalize to an infinite-temperature state, $\rho \propto \mathbb{I}$ for $t>t_{*}$, due to the corrections in Eq. (6) that cannot eventually be neglected, one refers to equilibration to $\rho_{\beta}$ in the preheating regime as "prethermalization." In particular, we can then talk about "prethermal quasiperiodically driven phases of matter" in this steady state. Of course, one must remember to include the effects of the time-quasiperiodic unitary $P(t)$ upon moving back to the laboratory frame. However, as $P(t)$, when constructed in the high-frequency limit, is perturbatively close to the identity, this simply endows the steady state $\rho_{\beta}$ with additional micromotion of small amplitude, $\sim J / \omega$; see Fig. 2.

We can also consider the case where $D$ is nonergodic, such as when it is highly disordered, leading to many-body localization (MBL). In this case, there is a complete set of quasilocal integrals of motion $\tau_{i}^{z}$ ( $l$-bits) satisfying $\left[\tau_{i}^{z}, D\right]=0$. The system evolving under $D$, when viewed in the rotating frame $P(t)$, will not thermalize but instead exhibits MBL phenomenology, such as logarithmic entanglement growth, initial state memory, and localizationprotected quantum order [74-77].

In the laboratory frame, the dynamics is rather more interesting. Owing to the rotating change of frame, the $l$ bits are not constants of motion but rather always evolve in time. Despite this motion, there is a sense in which the system is still localized: Consider the dressed $l$-bit operator 


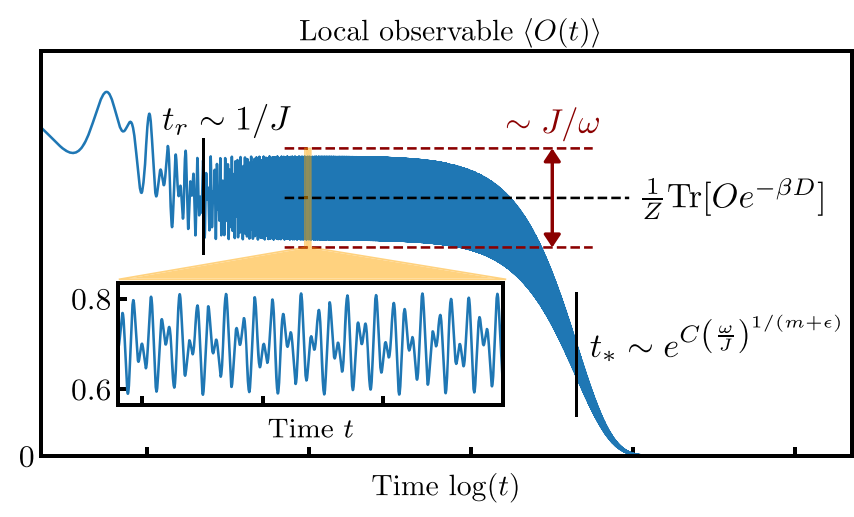

FIG. 2. Prethermalization in quasiperiodically driven systems at high frequencies. Shown is a cartoon of the dynamics of a generic traceless local observable $\langle O(t)\rangle$ under time evolution by Eq. (6). There are three regimes. First, a brief transient regime, where the local observable relaxes on a short timescale $t_{r} \sim 1 / J$, where $J$ is the local energy scale of the system. Second, a prethermalization regime, where the system has locally equilibrated to a thermal ensemble of an effective static Hamiltonian $D$, when viewed in the rotating frame defined by $P(t)$. The evolution $\langle O(t)\rangle$ in the laboratory frame shows a plateau around the prethermal value (black dashed line), with small time-quasiperiodic oscillations of amplitude of $\sim J / \omega$ (red dashed lines); here, $\omega=|\omega|$ is the norm of driving frequencies. This regime lasts up to the long heating time $t_{*} \sim \exp \left[C(\omega / J)^{1 /(m+\epsilon)}\right]$. Third, a final featureless infinitetemperature state, reached after the system has fully heated. The inset shows a zoom-in on the orange-shaded region in the prethermal plateau.

$\tilde{\tau}_{i}^{z}:=P(0) \tau_{i}^{z} P(0)^{\dagger}$, which is localized near site $i$. Under "reverse evolution" defined as

$$
\tilde{\tau}_{i}^{z}(t)_{R}=U(t) \tilde{\tau}_{i}^{z} U(t)^{\dagger}
$$

we obtain, using Eq. (6) and $\left[\tau_{i}^{z}, D\right]=0$, that

$$
\tilde{\tau}_{i}^{z}(t)_{R} \approx P(t) e^{-i t D} \tau_{i}^{z} e^{i t D} P(t)^{\dagger}=P(t) \tau_{i}^{z} P(t)^{\dagger} .
$$

This means that the motion of the $\tilde{\tau}_{i}^{z}(t)_{R}$ is time quasiperiodic, which in turn implies that there is an infinite sequence in time whereby the operator returns arbitrarily close (but never exactly) to the initial operator $\tilde{\tau}_{i}^{z}$. Why should the reverse evolution of the dressed $l$-bit $\tilde{\tau}_{i}^{z}$ be a useful concept? Consider the forward Heisenberg time evolution of an operator $O_{i}$ localized near site $i$ via $O_{i}(t):=$ $U(t)^{\dagger} O_{i} U(t)$ and ask how this operator spreads over time in space. In an ergodic system, we expect that any local operator spreads generically ballistically, or diffusively at the slowest. In our present case, computing the overlap of $O_{i}(t)$ with the localized dressed $l$-bit $\tilde{\tau}_{i}^{z}$,

$$
\operatorname{Tr}\left(O_{i}(t) \tilde{\tau}_{i}^{z}\right)=\operatorname{Tr}\left(O_{i} \tilde{\tau}_{i}^{z}(t)_{R}\right),
$$

reveals that this overlap varies quasiperiodically in time rather than decaying to zero. We can interpret this as the statement that some fraction of the operator $O_{i}(t)$ remains localized near its origin rather than being transported away.

What we have described is thus a new kind of dynamical localization that can be dubbed "quasiperiodically driven MBL," although we have only shown that it is stable until the timescale $t_{*}$ bounded by Eq. (3). Proving whether the quasiperiodically driven MBL is stable beyond this time, perhaps even forever, remains an interesting direction for future work.

In this paper, we consider quasiperiodically driven phases of matter realizable in one of the scenarios described above: prethermalization or (stretched-exponentially longlived) quasiperiodically driven MBL.

\section{EMERGENT SYMMETRIES PROTECTED BY MULTIPLE TIME-TRANSLATION SYMMETRIES}

Having motivated quasiperiodically driven systems and outlined their dynamics in suitable regimes, we now analyze what kinds of new phases of matter can arise in these systems. As a first step, let us consider the scenario where a direct high-frequency drive is applied to a system. This procedure is often referred to as "high-frequency Floquet engineering," as the drive is used to modify and control interactions of an underlying Hamiltonian. Indeed, the ground states of the effective static Hamiltonian $D$ that is generated in a high-frequency expansion can be different from those of the original undriven Hamiltonian [5,78-82].

However, from a phases-of-matter point of view, a direct high-frequency drive will not yield fundamentally new long-time collective behavior that is not already reproducible in some-possibly complicated-static system at equilibrium. In this regime, a quantum state's evolution is effectively governed entirely by $D$ and never has any significant nontrivial micromotion during its time evolution. Precisely, this result stems from the fact that the unitary frame transformation $P(t)$ in the description of the time-evolution operator, Eq. (6), is perturbatively close to the identity. To uncover novel phases, especially those that are inherently out of equilibrium, we need to go beyond this regime.

In order to do this, we generalize the idea of a frametwisted high-frequency limit, introduced in Ref. [12] for Floquet systems and reviewed in Sec. III A, to the quasiperiodically driven scenario. This generalized limit will be the context in which fundamentally new, long-lived phases of matter can emerge. In order to analyze the manifestation of TTSs in this regime, we introduce the notion of twisted time-translation symmetries (Sec. III B). This concept allows us to analyze the quasiperiodic case but also gives a simpler perspective on the results in the Floquet case compared to the original constructions of Ref. [12]. Finally, in Sec. III C, we explain how to realize these twisted timetranslation symmetries in a frame-twisted high-frequency limit in quasiperiodically driven systems. 


\section{A. Review: Frame-twisted high-frequency limit in Floquet systems}

For Floquet systems ( $m=1)$, Ref. [12] provided general periodic driving conditions that do give rise to fundamentally new nonequilibrium phases. We briefly review these conditions here.

The main idea is to consider periodically driven Hamiltonians that approach the high-frequency limit but only when viewed in a certain rotating frame, a so-called frametwisted high-frequency limit. Consider a many-body driven system with a Hamiltonian of the form $H(t)=$ $H_{0}(t)+V(t)$. Here, $H_{0}(t)=H_{0}(t+T)$ is a sum of quasilocal terms, with the associated time-evolution operator $U_{0}(t)=\mathcal{T} \exp \left[-i \int_{0}^{t} H_{0}\left(t^{\prime}\right) d t^{\prime}\right]$. Evolution under $H_{0}$ is taken to have the special property $U_{0}(N T)=U_{0}(T)^{N}=$ $X^{N}=\mathbb{I}$, for some positive integer $N>1$ and an operator $X$, which is not the identity. The term $V(t)=V(t+T)$ describes interactions assumed to have a local energy scale $J \ll \omega / N$. Since $X$ is not perturbatively accessible from the identity, a strong drive is required to realize this evolution-the local energy scale of $H_{0}$ is $\sim \omega / N$ and thus increases with $\omega$. One therefore cannot naively apply the high-frequency expansion in Eq. (5).

In the rotating frame defined by the interaction picture of $H_{0}(t)$, time evolution is governed by the interaction Hamiltonian

$$
H_{\text {int }}(t)=U_{0}^{\dagger}(t) V(t) U_{0}(t) .
$$

This Hamiltonian is quasilocal and still time periodic, albeit with period $N T$. Since $J \ll \omega / N$, a high-frequency expansion can be meaningfully applied to it, and one can see how there is a long-lived preheating regime in this frame of reference.

However, the frame-twisted high-frequency limit imposes a stronger condition than just long-lived preheating. Specifically, as shown in Ref. [12], in the laboratory frame, the Floquet unitary $U_{F}:=U(T)$ takes on the special structure

$$
U_{F} \approx \mathcal{V}\left(X e^{-i D T}\right) \mathcal{V}^{\dagger}
$$

where $D$ is a quasilocal Hamiltonian that additionally satisfies $[D, X]=0$ identically. Here, $\mathcal{V}$ is a time-independent quasilocal unitary that is perturbatively close to the identity $\mathbb{I}$. The approximate equality reflects an omission of small time-dependent local terms whose effects only become relevant after times $t_{*} \sim O\left(e^{\text {const } \omega / J}\right)$.

The physical statement is that a system periodically driven under these conditions always has an emergent $\mathbb{Z}_{N}$ symmetry generated by $X$. One can add small, potentially time-dependent perturbations to $H(t)$ as long as they respect the time-periodic nature of the drive, and the structure of Eq. (12) will be unchanged. The emergent symmetry is therefore robust and underpins the stability of inherently nonequilibrium Floquet phases of matter in many-body systems that can now emerge. From Eq. (12), one sees that when observed at times $t$ that are integer multiples of $N T$ (i.e., at times that are stroboscopic with respect to the longer period), the system, in the time-independent frame described by $\mathcal{V}$, settles into an equilibrium state of $D$ distinguished by the emergent $\mathbb{Z}_{N}$ symmetry. However, when viewed after every time interval $T$ (the original period of the driving Hamiltonian), because of the action of the symmetry operator $X$, the state of the system can transform nontrivially. This transformation happens, for example, if the system spontaneously breaks the symmetry. Note that both the concepts of equilibration and spontaneous symmetry breaking are only sharply defined in a thermodynamically large system. This additional periodic action is precisely what makes the longtime collective behavior of this system inherently out of equilibrium, and the robustness of the phenomenology justifies the terminology of them being called fundamentally nonequilibrium phases of matter. We reiterate that this remarkable result is a consequence of the discrete TTS of the Floquet drive, and it is guaranteed to occur with no additional symmetry requirements.

\section{B. Twisted time-translation symmetries and emergent symmetries}

In quasiperiodically driven $(m \geq 2)$ systems, it is natural to look for an expression of the form in Eq. (12). Since there is no single time-translation symmetry, however, there is no analog of the Floquet operator $U_{F}$, and the construction of Ref. [12] does not carry over.

Our key observation is that one can rederive the results of Ref. [12] for Floquet systems in a considerably simpler way that accords an extension to quasiperiodically driven systems. This approach relies on realizing that the interaction Hamiltonian $H_{\text {int }}(t)$ described in the previous section possesses a symmetry as a consequence of the TTS of the original laboratory-frame Hamiltonian. We refer to this symmetry of $H_{\text {int }}(t)$ as a twisted time-translation symmetry.

Precisely, in the Floquet setting, we say that a timeperiodic operator $O(t)=O(t+T)$ has a twisted TTS, if there is an integer $N>1$ and a unitary operator $g$ satisfying $g^{N}=\mathbb{I}$, such that for all $t$,

$$
O(t+\tilde{T})=g O(t) g^{\dagger},
$$

where $\tilde{T}=T / N$. In terms of the Fourier modes $O_{n}$ in $O(t)=\sum_{n} O_{n} e^{i n \omega t}$, the twisted TTS states that $g O_{n} g^{\dagger}=$ $e^{2 \pi i n / N} O_{n}$. Note that $H_{\text {int }}(t)$ in Eq. (11) has a twisted TTS with unitary $g=X^{\dagger}$, provided we rescale time $t \mapsto t / N$ so that the periodicity of $H_{\mathrm{int}}(t)$, originally $N T$, becomes $T$.

To gain some intuition as to why this concept is useful, suppose that we have a Hamiltonian $H(t)$ with a twisted TTS, and we construct the effective Hamiltonian $D$ from the high-frequency expansion given by Eq. (7) with $m=1$. 
One immediately sees from the action of twisted TTS in Fourier space that $D$ constructed in this way commutes with $g$ to all orders. Additionally, it can be shown that the change of frame $P(t)$ will also inherit the same twisted TTS as $H(t)$, i.e., $P(t+\tilde{T})=g P(t) g^{\dagger}$. Note that the usual Floquet-Magnus expansion in Eq. (5) will not give this result; see Appendix A. The physical conclusion is that dynamics of a driven Hamiltonian with a twisted TTS can always be viewed in some frame as effectively governed by a time-independent Hamiltonian $D$ with an emergent $\mathbb{Z}_{N}$ internal symmetry, generated by $g$.

Applying these considerations to the rotating-frame Hamiltonian $H_{\text {int }}(t)$ in Eq. (11) to construct the effective Hamiltonian $D$ using Eq. (7), ones recovers the statement [Eq. (12)] of Ref. [12] in a transparent fashion:

Since $U_{F}=U_{0}(T) U_{\mathrm{int}}(T)=X \mathcal{T} e^{-i \int_{0}^{T} H_{\mathrm{int}}\left(t^{\prime}\right) d t^{\prime}}$, we can write it as

$$
U_{F} \approx X P(T) e^{-i D T} P(0)^{\dagger}=P(0)\left(X e^{-i D T}\right) P(0)^{\dagger},
$$

with $[D, X]=0$, and where we have used $X P(T)=P(0) X$.

The twisted TTS concept immediately generalizes to quasiperiodically driven systems $(m \geq 2)$. Recall that a time-quasiperiodic operator $O(t)=O\left(\omega t+\boldsymbol{\theta}_{0}\right)$ is derived from an operator $O(\boldsymbol{\theta})$ that is parametrized by a variable $\boldsymbol{\theta}$ living in a higher-dimensional space, where $O(\boldsymbol{\theta}+\boldsymbol{\tau})=$ $O(\boldsymbol{\theta})$ for any $\boldsymbol{\tau} \in \mathcal{L}=2 \pi \mathbb{Z}^{m}$. Suppose there is additionally some finite translation vector $\tilde{\boldsymbol{\tau}}$ and a unitary operator $g_{\tilde{\boldsymbol{\tau}}}$ satisfying $g_{\tilde{\tau}}^{N}=\mathbb{I}$ for some integer $N>1$, such that

$$
O(\boldsymbol{\theta}+\tilde{\boldsymbol{\tau}})=g_{\tilde{\tau}} O(\boldsymbol{\theta}) g_{\tilde{\tau}}^{\dagger} .
$$

We then say that $O(\boldsymbol{\theta})$ has a $g_{\tilde{\tau}}$-twisted time-translation symmetry. Note that as $O(\boldsymbol{\theta}+N \tilde{\boldsymbol{\tau}})=O(\boldsymbol{\theta}), N \tilde{\boldsymbol{\tau}} \in \mathcal{L}$. In terms of Fourier modes $O_{n}$, the twisted TTS acts as $g_{\tilde{\tau}} O_{n} g_{\tilde{\tau}}^{\dagger}=e^{i n \cdot \tilde{\tau}} O_{n}$. Furthermore, since $O(\boldsymbol{\theta})$ is defined on a torus with dimension $m \geq 2$, it can have multiple independent twisted TTSs corresponding to different translation vectors $\tilde{\boldsymbol{\tau}}$ and unitary operators $g_{\tilde{\tau}}$.

As in the Floquet case, for a Hamiltonian with twisted TTSs, the effective Hamiltonian $D$ constructed through Eq. (7) manifestly commutes with $g_{\tilde{\tau}}$. Also, $P(\boldsymbol{\theta})$ will inherit the same twisted TTSs $P(\boldsymbol{\theta}+\tilde{\boldsymbol{\tau}})=g_{\tilde{\tau}} P(\boldsymbol{\theta}) g_{\tilde{\tau}}^{\dagger}$. These properties will hold for other high-frequency expansions as well, like the one we develop in Sec. VII.

\section{Frame-twisted high-frequency limit in quasiperiodically driven systems}

Although Eq. (14) seems like an obscure condition, analogous to the Floquet case, twisted TTSs can arise naturally in a frame-twisted high-frequency limit of a drive that does not need to satisfy additional symmetry constraints beyond time quasiperiodicity itself. Indeed, we show how $m$ twisted TTSs can manifest from $m$ "untwisted" time translations of the original driving Hamiltonian $H(\boldsymbol{\theta})$, when viewed in a suitable frame of reference.

As discussed in Sec. II A, the many-body Hamiltonian of the system $H(t)=H\left(\boldsymbol{\omega} t+\boldsymbol{\theta}_{0}\right)$ derives from a Hamiltonian $H(\boldsymbol{\theta})$ on the standard torus $\mathbb{T}^{m}$. Assume now that this Hamiltonian has the form

$$
H(\boldsymbol{\theta})=H_{0}(\boldsymbol{\theta})+V(\boldsymbol{\theta}),
$$

with $H_{0}(\boldsymbol{\theta}+\boldsymbol{\tau})=H_{0}(\boldsymbol{\theta})$ and $V(\boldsymbol{\theta}+\boldsymbol{\tau})=V(\boldsymbol{\theta})$, for any $\tau \in \mathcal{L}=2 \pi \mathbb{Z}^{m}$. Let $\Gamma_{1}, \ldots, \Gamma_{r}$ be a set of $r$ mutually commuting operators, each of which is a sum of quasilocal terms and has integer eigenvalues. We take

$$
H_{0}(\boldsymbol{\theta})=f_{i}(\boldsymbol{\theta}) \Gamma_{i} .
$$

Unless otherwise stated, the index summation convention is implied. Here, $f_{i}(\boldsymbol{\theta})$ are real-valued functions satisfying $\bar{f}_{i}:=\int_{\mathbb{T}^{m}}\left[d^{m} \boldsymbol{\theta} /(2 \pi)^{m}\right] f_{i}(\boldsymbol{\theta})=Q_{i j} \omega_{j}$, where $Q_{i j}$ is a dimensionless $r \times m$ matrix with rational entries. The interaction term $V(\boldsymbol{\theta})$ is a quasilocal Hamiltonian with local energy scale $J$.

Under these conditions, we can solve for the evolution operator $U_{0}(t)=\mathcal{T} \exp \left[-i \int_{0}^{t} H_{0}\left(\boldsymbol{\theta}_{0}+\boldsymbol{\omega} t^{\prime}\right) d t^{\prime}\right]$. Since it is made from commuting terms, the time ordering can be neglected. It is quasiperiodic, $U_{0}(t)=U_{0}\left(\boldsymbol{\omega} t+\boldsymbol{\theta}_{0}\right)$, and can be expressed as

$$
U_{0}(\boldsymbol{\theta})=e^{-i h_{i}(\boldsymbol{\theta}) \Gamma_{i}},
$$

for some functions $h_{i}(\boldsymbol{\theta})$ satisfying $h_{i}(\boldsymbol{\theta}+\boldsymbol{\tau})=h_{i}(\boldsymbol{\theta})+$ $Q_{i j} \tau_{j}$ for all $\tau \in \mathcal{L}$ (see Appendix B).

Notice that $U_{0}(\boldsymbol{\theta})$ is defined on a larger torus than $H_{0}(\boldsymbol{\theta})$. Specifically:

$$
U_{0}\left(\boldsymbol{\theta}+\boldsymbol{\tau}^{\prime}\right)=U_{0}(\boldsymbol{\theta}), \quad \text { for all } \boldsymbol{\tau}^{\prime} \in \mathcal{L}^{\prime} .
$$

Here, $\mathcal{L}^{\prime}$ is a sublattice of the original lattice $\mathcal{L}$ defined by $\boldsymbol{\tau}^{\prime} \in \mathcal{L}^{\prime}$ if and only if $\boldsymbol{\tau}^{\prime} \in \mathcal{L}$ and $e^{i Q_{i j} \tau_{j}^{\prime}}=1$. A simple example is where $m=r$ and $Q=(1 / N) \mathbb{I}_{m}$ for some integer $N>1$; in that case, $\mathcal{L}^{\prime}=2 \pi N \mathbb{Z}^{m}=N \mathcal{L}$, so the basic original cell is enlarged by $N$ in each direction. We emphasize that Eq. (18) holds only due to our special form of $H_{0}(t)$ from Eq. (16). In general, one does not expect $U_{0}(t)$ to be quasiperiodic, even if $H_{0}(t)$ is.

We are now in a position to see how twisted TTSs emerge. By transforming into the interaction picture of $H_{0}(t)$, the interaction Hamiltonian is

$$
H_{\mathrm{int}}\left(\boldsymbol{\omega} t+\boldsymbol{\theta}_{0}\right)=U_{0}^{\dagger}(t)\left(H(t)-i \partial_{t}\right) U_{0}(t)
$$

and has local energy scale $J$. Furthermore, it derives from a Hamiltonian $H_{\text {int }}(\boldsymbol{\theta})=U_{0}^{\dagger}(\boldsymbol{\theta}) V(\boldsymbol{\theta}) U_{0}(\boldsymbol{\theta})$, which has periodicity in the larger unit cell $H_{\text {int }}\left(\boldsymbol{\theta}+\boldsymbol{\tau}^{\prime}\right)=H_{\text {int }}(\boldsymbol{\theta})$, with 


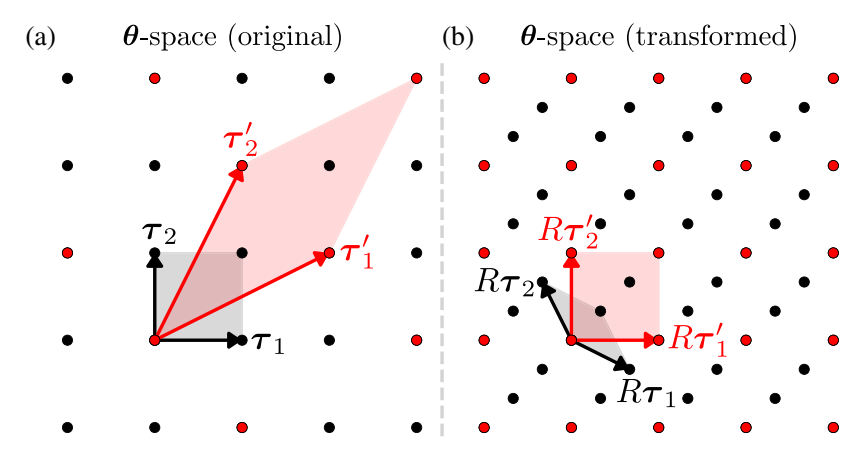

FIG. 3. Twisted TTS and action of linear transformation $R$ on $\boldsymbol{\theta}$ space for a specific $m=2$ example. (a) The lattice $\mathcal{L}$ of points (black) is defined by the translation vectors $\tau_{1}=2 \pi(1,0)$ and $\boldsymbol{\tau}_{2}=2 \pi(0,1)$. The original driving Hamiltonian $H(\boldsymbol{\theta})$, Eq. (15), has periodicity on the standard torus (grey shaded area). The interaction Hamiltonian $H_{\text {int }}(\boldsymbol{\theta})$ generally has different periodicity. We show an example where the periodicity is on a sheared torus (red shaded area), given by the translation vectors $\boldsymbol{\tau}_{1}^{\prime}=2 \pi(2,1)$ and $\boldsymbol{\tau}_{2}^{\prime}=2 \pi(1,2)$, which defines a lattice $\mathcal{L}^{\prime}$ (red points). (b) Invertible linear transformation $R$ mapping $\boldsymbol{\theta} \mapsto R \boldsymbol{\theta}$ so that $\left(\boldsymbol{\tau}_{1}^{\prime}, \boldsymbol{\tau}_{2}^{\prime}\right) \mapsto\left(R \boldsymbol{\tau}_{1}^{\prime}, R \boldsymbol{\tau}_{2}^{\prime}\right)=\left(\boldsymbol{\tau}_{1}, \boldsymbol{\tau}_{2}\right)$. The Hamiltonian $H_{\text {int }}^{\prime}(\boldsymbol{\theta}):=H_{\text {int }}\left(R^{-1} \boldsymbol{\theta}\right)$ has periodicity on the standard torus (red shaded area) and additionally has $g_{\tau}$ twisted TTSs $H_{\mathrm{int}}^{\prime}(\boldsymbol{\theta}+R \boldsymbol{\tau})=g_{\boldsymbol{\tau}} H_{\mathrm{int}}^{\prime}(\boldsymbol{\theta}) g_{\boldsymbol{\tau}}^{\dagger}$, where $\boldsymbol{\tau} \in 2 \pi \mathbb{Z}^{2}$.

$\tau^{\prime} \in \mathcal{L}^{\prime}$. Choosing $Q$ to have rational entries ensures that the new unit cell is still finite (see Appendix C).

Since the $\Gamma_{i}$ commute, Eq. (17) implies $U_{0}(\boldsymbol{\theta}+\boldsymbol{\tau})^{\dagger}=$ $g_{\boldsymbol{\tau}} U_{0}(\boldsymbol{\theta})^{\dagger}=U_{0}(\boldsymbol{\theta})^{\dagger} g_{\boldsymbol{\tau}}$, where

$$
g_{\tau}=\exp \left(i Q_{i j} \Gamma_{i} \tau_{j}\right)
$$

for $\boldsymbol{\tau} \in \mathcal{L}$. Together with $V(\boldsymbol{\theta})=V(\boldsymbol{\theta}+\boldsymbol{\tau})$, this result yields

$$
H_{\mathrm{int}}(\boldsymbol{\theta}+\boldsymbol{\tau})=g_{\boldsymbol{\tau}} H_{\mathrm{int}}(\boldsymbol{\theta}) g_{\boldsymbol{\tau}}^{\dagger}
$$

This equation is almost the twisted TTS condition in Eq. (14), except the periodicity of $H_{\text {int }}(\boldsymbol{\theta})$ is not on the standard torus $\mathbb{T}^{m}$. However, there is always an invertible linear transformation $R$ on $\boldsymbol{\theta}$ space (Fig. 3), so that the Hamiltonian $H_{\text {int }}^{\prime}(\boldsymbol{\theta}):=H_{\text {int }}\left(R^{-1} \boldsymbol{\theta}\right)$ is periodic on the standard torus and Eq. (14) then holds exactly for $H_{\mathrm{int}}^{\prime}(\boldsymbol{\theta})$.

Generally, we do not need to invoke the transformation $R$, except when we discuss estimates of heating times and our construction of effective Hamiltonians in the preheating regime, taking as input high-frequency Hamiltonians that are periodic on the standard torus. In those cases, we should remember that the coordinate transformation means that the frequency vector is also rescaled $\omega \mapsto R \omega$ when we consider the single-time evolution since $H_{\text {int }}\left(\boldsymbol{\omega} t+\boldsymbol{\theta}_{0}\right)=$ $H_{\text {int }}^{\prime}\left(R \boldsymbol{\omega} t+R \boldsymbol{\theta}_{0}\right)$. This implies that the dynamics governed by the effective Hamiltonian $D$ constructed from $H_{\text {int }}^{\prime}(\boldsymbol{\theta})$ in a high-frequency expansion assuming $J \ll R_{i j} \omega_{j}$ will last for a long time, $t_{*} \sim O\left(e^{\operatorname{const} \times(|R \omega| / J)^{1 /(m+\epsilon)}}\right)$; see Sec. VII. Then, for times less than $t_{*}$, the evolution operator in the interaction frame can be written as

$$
U_{\mathrm{int}}(t) \approx P\left(\boldsymbol{\omega} t+\boldsymbol{\theta}_{0}\right) e^{-i D t} P^{\dagger}\left(\boldsymbol{\theta}_{0}\right),
$$

where $P(\boldsymbol{\theta})$ is periodic with respect to translations $\boldsymbol{\tau}^{\prime} \in \mathcal{L}^{\prime}$.

According to the discussion of Sec. III B, the effective Hamiltonian $D$ in the preheating regime will have emergent symmetries arising from the twisted TTSs,

$$
\left[D, g_{\tau}\right]=0, \quad \text { for all } \boldsymbol{\tau} \in \mathcal{L} .
$$

Moreover, $P(\boldsymbol{\theta}+\boldsymbol{\tau})=g_{\boldsymbol{\tau}} P(\boldsymbol{\theta}) g_{\boldsymbol{\tau}}^{\dagger}$ for $\boldsymbol{\tau} \in \mathcal{L}$. Analogous to the Floquet case, these symmetry properties of $D$ are robust to small, potentially time-dependent perturbations to the driving protocol, as long as they respect the time quasiperiodicity of the system.

Although Eq. (23) holds for each translation vector $\tau \in \mathcal{L}$, not every such $\tau$ corresponds to a different operator $g_{\boldsymbol{\tau}}$. In fact, $g_{\boldsymbol{\tau}}=\mathbb{I}$ if and only if $\boldsymbol{\tau} \in \mathcal{L}^{\prime}$. The unitary operators $g_{\tau}$ therefore belong to a finite Abelian group of emergent symmetries, $\mathcal{G} \cong \mathcal{L} / \mathcal{L}^{\prime}$. In the simple case where $Q=(1 / N) \mathbb{I}_{m}$, we find that $m=r, \mathcal{G}=\mathbb{Z}_{N}^{\times m}$. As a slightly less trivial example, consider $r=m=2$ and $Q=\frac{1}{3}\left(\begin{array}{rr}-1 & 2 \\ 2 & 1\end{array}\right)$, which results in a lattice $\mathcal{L}^{\prime}$ as seen in Fig. 3, and $\mathcal{G}=\mathbb{Z}_{3}$. We refer the reader to Appendix C where we show, given some rational matrix $Q$, how to compute $\mathcal{G}$ from the Smith decomposition of $Q$. Since $\mathcal{G}$ is a finite Abelian group, it is always of the form $\mathcal{G}=\mathbb{Z}_{q_{1}} \times$ $\mathbb{Z}_{q_{2}} \times \ldots$ [83].

Collecting all the ingredients discussed above, we are now in the position to realize novel, inherently out-ofequilibrium phases of matter. Time evolution in the laboratory frame for $t<t_{*}$, under the driving scenarios outlined in this section is governed by the evolution operator $U(t)=U_{0}(t) U_{\text {int }}(t)$, which can be written as

$$
\begin{aligned}
U(t) & \approx U_{0}\left(\boldsymbol{\omega} t+\boldsymbol{\theta}_{0}\right) P\left(\boldsymbol{\omega} t+\boldsymbol{\theta}_{0}\right) e^{-i D t} P^{\dagger}\left(\boldsymbol{\theta}_{0}\right) \\
& =\mathcal{V}(t)\left[U_{0}(t) e^{-i D t}\right] \mathcal{V}^{\dagger}(0)
\end{aligned}
$$

Here, $\mathcal{V}(t)=U_{0}\left(\boldsymbol{\omega} t+\boldsymbol{\theta}_{0}\right) P\left(\boldsymbol{\omega} t+\boldsymbol{\theta}_{0}\right) U_{0}^{\dagger}\left(\boldsymbol{\omega} t+\boldsymbol{\theta}_{0}\right)$ is time quasiperiodic with underlying $\mathcal{V}(\boldsymbol{\theta})=U_{0}(\boldsymbol{\theta}) P(\boldsymbol{\theta}) U_{0}^{\dagger}(\boldsymbol{\theta})$ that has periodicity on the standard torus $\mathbb{T}^{m}$, that is, for translations $\tau \in \mathcal{L}$. Another way to state Eq. (24) is that, in the rotating frame defined by $\mathcal{V}(t) U_{0}(t)$, time evolution of the system is simply governed effectively by the static Hamiltonian $D$. However, if one goes back to the laboratory frame, this frame transformation, which is not perturbative close to the identity, can endow the state of the system (in particular, the steady state of $D$ ) with large, structured, time-quasiperiodic micromotion, giving rise to a panoply of different long-time dynamical collective behaviors whose dynamical signatures are robust and universal. 
In the next two sections, we illustrate the physical implications of our results with two examples of such phases: time quasicrystals and dynamic quasiperiodic topological phases. We return to the important task of formalizing the preceding discussions on dynamics, as well as explicitly constructing $D$, in later sections.

\section{DISCRETE TIME QUASICRYSTALS}

A discrete time quasicrystal (DTQC) is a phase that spontaneously breaks some or all of the time-translation symmetries of a quasiperiodic drive [64]. It is characterized by a dynamical response of physical observables, which display stable long-time oscillations with a time quasiperiodicity that is different from the time quasiperiodicity of the original driving Hamiltonian $H(t)$. This difference in quasiperiodicity can be diagnosed by computing the power spectra of local observables, which will exhibit robust peaks at frequencies that are shifted from the base frequencies by a fractional amount. Since there are several $(m \geq 2)$ independent time-translation symmetries, there are a multitude of ways that these symmetries can be spontaneously broken, leading to a variety of patterns and associated DTQC phases. The DTQC generalizes the discrete time crystal, a phase that spontaneously breaks the single timetranslation symmetry of Floquet systems [9,11].

Note that the concept of a DTQC as well as some aspects of its phenomenology have been proposed in Ref. [64], which numerically observed a DTQC-like signal in a quasiperiodic step drive with disorder, albeit with a slow logarithmic decay of the envelope in time. Our present work explains, more generally, the precise role of time-translational symmetries of quasiperiodic drives in delineating such a phase, and it also shows how the logarithmic decay can be avoided (even without disorder) through smooth driving, hence rigorously proving the stability of the phase up to the long heating time $t_{*}$. In addition, we provide drives that generalize to a large class of symmetry-breaking patterns. This large class includes, among many others, the DTQC pattern introduced in Ref. [64], as well as that of Ref. [66], and we address the stability of such patterns when using smooth driving.

To understand DTQC phases, consider the time evolution of a quantum state $\left|\psi_{0}\right\rangle$ with a quasiperiodic Hamiltonian of the type discussed in Sec. III C. Then, one can take a frametwisted high-frequency limit, so for times $t<t_{*}$, the timeevolution operator can be decomposed as in Eq. (24). Recall that the effective time-independent Hamiltonian $D$ in the preheating regime possesses multiple unitary symmetries $g_{\tau}$, where $\tau \in \mathcal{L}=2 \pi \mathbb{Z}^{m}$, which belong to some finite Abelian group $\mathcal{G}$.

Now, let us consider times where the system has prethermalized, so in the rotating frame $\mathcal{V}(t) U_{0}(t)$, the state is locally described by a thermal state $\rho_{\beta}$; see the discussion in Sec. II D. In the laboratory frame, the state of the system when probed by local observables is $\rho(t)=\rho\left(\boldsymbol{\omega} t+\boldsymbol{\theta}_{0}\right)$, where

$$
\rho(\boldsymbol{\theta})=\mathcal{V}(\boldsymbol{\theta}) U_{0}(\boldsymbol{\theta}) \rho_{\beta} U_{0}^{\dagger}(\boldsymbol{\theta}) \mathcal{V}^{\dagger}(\boldsymbol{\theta})
$$

We see that $\rho(t)$ is time quasiperiodic since $\rho(\boldsymbol{\theta})$ at least satisfies $\rho\left(\boldsymbol{\theta}+\boldsymbol{\tau}^{\prime}\right)=\rho(\boldsymbol{\theta})$ for $\boldsymbol{\tau}^{\prime} \in \mathcal{L}^{\prime}$. But does $\rho(\boldsymbol{\theta})$ have the periodicity of the original drive $H(\boldsymbol{\theta})$ of Eq. (15), characterized by the lattice $\mathcal{L}=2 \pi \mathbb{Z}^{m}$ ? The answer depends on whether or not $\rho_{\beta}$ is symmetric under the emergent symmetries $g_{\tau}$.

To see this explicitly, we can write

$$
\rho(\boldsymbol{\theta}+\boldsymbol{\tau})=\mathcal{V}(\boldsymbol{\theta}) U_{0}(\boldsymbol{\theta}) g_{\tau}^{\dagger} \rho_{\beta} g_{\boldsymbol{\tau}} U_{0}^{\dagger}(\boldsymbol{\theta}) \mathcal{V}^{\dagger}(\boldsymbol{\theta}),
$$

where we use $\mathcal{V}(\boldsymbol{\theta}+\boldsymbol{\tau})=\mathcal{V}(\boldsymbol{\theta})$ and $U_{0}(\boldsymbol{\theta}+\boldsymbol{\tau})=U_{0}(\boldsymbol{\theta}) g_{\boldsymbol{\tau}}^{\dagger}$. Therefore, $\rho(\boldsymbol{\theta}+\boldsymbol{\tau})=\rho(\boldsymbol{\theta})$ for $\boldsymbol{\tau} \in \mathcal{L}$ if and only if $g_{\tau} \rho_{\beta} g_{\tau}^{\dagger}=\rho_{\beta}$. In other words, if $\rho_{\beta}$ is a state that preserves all the symmetries $g_{\tau}$ of the effective Hamiltonian $D$, then $\rho(\boldsymbol{\theta})$ preserves all $m$ multiple time-translation symmetries of the driving Hamiltonian $H(\boldsymbol{\theta})$.

If, on the other hand, $\rho_{\beta}$ is not invariant under $g_{\tau}$, $g_{\tau} \rho_{\beta} g_{\tau}^{\dagger} \neq \rho_{\beta}$, then the emergent symmetry $g_{\tau}$ is said to be spontaneously broken in the thermal state of $D$. This property can, of course, happen for multiple $g_{\tau}$ at the same time. From Eq. (26), $\rho(\boldsymbol{\theta})$ will then have a periodicity different from the original Hamiltonian $H(\boldsymbol{\theta})$, and consequently, $\rho(t)=\rho\left(\boldsymbol{\omega} t+\boldsymbol{\theta}_{0}\right)$ will have a different time quasiperiodicity than the driving Hamiltonian $H(t)=H\left(\boldsymbol{\omega} t+\boldsymbol{\theta}_{0}\right)$ - the hallmark of a DTQC phase. The precise connection between the spontaneous breaking of $g_{\boldsymbol{\tau}}$ and of TTS reflects the fact that $g_{\boldsymbol{\tau}}$ is a manifestation of the TTS in the first place.

\section{A. Observable consequences}

The spontaneously broken TTSs in quasiperiodically driven systems manifest themselves most clearly through periodicity changes in the $m$-dimensional $\boldsymbol{\theta}$ space. The interplay of multiple time-translation symmetries gives a large variation in the number of different symmetrybreaking patterns and associated DTQC phases. However, there are also measurable signatures of these patterns in terms of the dependence of the system on physical time $t$, for example, in the Fourier spectrum (or power spectrum) of local observables (see also Ref. [64]). These are analogous to probing quasicrystalline structures in space through their diffraction patterns $[84,85]$.

Consider the regime described above, where the state of the system is described by Eq. (26). Then, the expectation of a local observable $o(t):=\langle\hat{o}(t)\rangle$ can be written as $o(t)=o\left(\boldsymbol{\omega} t+\boldsymbol{\theta}_{0}\right)$, where $o(\boldsymbol{\theta}):=\operatorname{Tr}(\hat{o} \rho(\boldsymbol{\theta}))$ has periodicity that depends on the periodicity of $\rho(\boldsymbol{\theta})$. Let $\mathcal{L}_{\mathrm{SSB}}$ be the sublattice of $\mathcal{L}$ comprising those $\boldsymbol{\tau} \in \mathcal{L}$ such that $\rho(\boldsymbol{\theta}+$ $\boldsymbol{\tau})=\rho(\boldsymbol{\theta})$ for all $\boldsymbol{\theta} ; \mathcal{L}_{\mathrm{SSB}}$ describes the symmetry-breaking pattern in $\boldsymbol{\theta}$ space. Then, we can expand $o(\boldsymbol{\theta})$ as a Fourier series 


$$
o(\boldsymbol{\theta})=\sum_{\boldsymbol{\alpha} \in \mathcal{L}_{\mathrm{SSB}}^{*}} e^{i \alpha \cdot \boldsymbol{\theta}} o_{\alpha}
$$

where the sum is over the reciprocal lattice vectors $\boldsymbol{\alpha} \in \mathcal{L}_{\mathrm{SSB}}^{*}$, which are the vectors $\boldsymbol{\alpha}$ satisfying $e^{i \alpha \cdot \tau}=1$ for all $\boldsymbol{\tau} \in \mathcal{L}_{\mathrm{SSB}}$. Consequently, the power spectrum of $o(t)$,

$$
\mathcal{P}_{o}(\Omega)=\sum_{\boldsymbol{\alpha} \in \mathcal{L}_{\mathrm{SSB}}^{*}}\left|o_{\alpha}\right|^{2} \delta(\Omega-\boldsymbol{\alpha} \cdot \boldsymbol{\omega}),
$$

has peaks at frequencies $\Omega_{\boldsymbol{\alpha}}=\boldsymbol{\alpha} \cdot \boldsymbol{\omega}$. Note that for smooth driving, the Fourier coefficient $o_{\alpha}$ will decay exponentially with $|\boldsymbol{\alpha}|$; furthermore, not every peak will necessarily appear for any choice of observable $\hat{o}$ since it is possible that $o_{\alpha}=0$ for some $\boldsymbol{\alpha}$ 's.

Now, in a DTQC, since the symmetry lattice $\mathcal{L}$ spontaneously breaks to the proper sublattice $\mathcal{L}_{\mathrm{SSB}}$, the reciprocal lattice $\mathcal{L}_{\mathrm{SSB}}^{*}$ is also a proper superlattice of $\mathcal{L}^{*}=\mathbb{Z}^{m}$. This implies that some of the frequencies $\Omega_{\alpha}=\boldsymbol{\alpha} \cdot \boldsymbol{\omega}$ for $\boldsymbol{\alpha} \in$ $\mathcal{L}_{\mathrm{SSB}}^{*}$ are not derivable from integer linear combinations of the base driving frequencies $\left(\omega_{1}, \ldots, \omega_{m}\right)$; i.e., they do not correspond to the base harmonics $\Omega_{\boldsymbol{n}}=\boldsymbol{n} \cdot \boldsymbol{\omega}$ for $\boldsymbol{n} \in \mathbb{Z}^{m}$, which is the dynamical signature of the spontaneous breaking of time-translation symmetries. Of course, the frequencies associated with original drive harmonics $\Omega_{n}=$ $\boldsymbol{n} \cdot \boldsymbol{\omega}$ are dense on the real line, so they can lie arbitrarily close to those frequencies $\Omega_{\alpha}$ reflecting the symmetry breaking. However, the peaks in the power spectrum at frequencies $\Omega_{\alpha}$ will nevertheless be well resolved from those at $\Omega_{n}$. This occurs because the weights of peaks at frequencies $\Omega_{n}$ approaching $\Omega_{\alpha}$ become ever more strongly suppressed as a consequence of the smoothness of the drive, as they involve very large $|\boldsymbol{n}|$. Therefore, the presence of well-defined peaks at frequencies $\Omega_{\alpha}$ constitutes a sharp dynamical signature of the DTQC phase. We discuss this point in greater depth in Sec. IV D, where we also discuss the effect of finite observation time (fundamentally limited by the heating time $t_{*}$ ) in resolving these peaks in practice.

Additionally, an important signature of the DTQC phase is that the location of these peaks $\Omega_{\alpha}$ in the power spectrum reflecting the spontaneous symmetry breaking is robust against small perturbations to the driving protocol, such as in changing $f(\boldsymbol{\theta}) \mapsto f(\boldsymbol{\theta})+\epsilon(\boldsymbol{\theta})$ for small, smooth $\epsilon(\boldsymbol{\theta})$, or by adding small time-quasiperiodic terms to the Hamiltonian $V$.

In the following subsection as well as Appendix D, we provide examples of Hamiltonians that exhibit DTQC phases.

\section{B. Example Hamiltonian: $\mathbb{Z}_{2}$ DTQC}

Consider a system of spin- $1 / 2$ degrees of freedom on a lattice, evolving with the $m=2$ time-quasiperiodic Hamiltonian

$$
\begin{gathered}
H(t)=H_{0}(\boldsymbol{\omega} t)+\sum_{i, j} J_{i j} \sigma_{i}^{z} \sigma_{j}^{z}+\sum_{i} h \sigma_{i}^{z}, \\
H_{0}(\boldsymbol{\theta})=\sum_{i} f(\boldsymbol{\theta}) \frac{1}{2}\left(\sigma_{i}^{x}+1\right) .
\end{gathered}
$$

Here, $\sigma^{x}, \sigma^{y}, \sigma^{z}$ are the standard Pauli matrices, and we choose the couplings $J_{i j}$ to be ferromagnetic so that the Ising Hamiltonian $\sum_{i, j} J_{i j} \sigma_{i}^{z} \sigma_{j}^{z}$ has an ordered phase at finite temperature. We also assume that the couplings decay sufficiently fast enough with spatial distance. For example, we can consider short-range nearest-neighbor couplings in two or greater dimensions, or power-law decaying interactions in one dimension with an exponent between one and two; see Sec. VIII A for a discussion of the dynamical consequence of power-law interactions. Note that we could replace $\sigma_{i}^{x}+1$ with $\sigma_{i}^{x}$ in Eq. (30) without affecting the dynamics, but we choose the former to ensure that $\frac{1}{2}\left(\sigma_{i}^{x}+1\right)$ has integer eigenvalues.

The Hamiltonian Eq. (29) falls into the class of Hamiltonians described in Sec. III C. It is comprised of two terms: First, $V$ describes pairwise Ising interactions between spins with amplitude $J_{i j}$, as well as a longitudinal field in the $z$ direction with strength $h$. The couplings are assumed to satisfy $J_{i j}, h \ll\left|\omega_{1}-\omega_{2}\right| / 2$. Second, $H_{0}(\boldsymbol{\omega} t)$ describes a quasiperiodic drive on the system in the $x$ direction, with frequency vector $\boldsymbol{\omega}=\left(\omega_{1}, \omega_{2}\right)$. In experimental platforms where such interactions can be realized, such as with trapped ions or ensembles of nitrogen-vacancy centers in diamond (see Sec. VIIIE), this drive can be implemented, for example, by external pulses using lasers or microwaves.

Let us now consider how to choose the driving profile $f(\boldsymbol{\theta})$. A natural generalization of models of the DTC previously considered in the Floquet case $[9,11,86,87]$ would be to take

$$
\begin{gathered}
f\left(\theta_{1}, \theta_{2}\right)=\pi\left[\omega_{1} \Delta\left(\theta_{1}\right)+\omega_{2} \Delta\left(\theta_{2}\right)\right], \\
\Delta(\theta)=\sum_{n=-\infty}^{\infty} \delta(\theta-\pi+2 \pi n) .
\end{gathered}
$$

If we substitute into Eqs. (29) and (30), we see that this drive corresponds to instantaneously applying $\sigma^{x}$ to all the spins at certain times $t$, namely, those for which either $\omega_{1} t-\pi$ or $\omega_{2} t-\pi$ is an integer multiple of $2 \pi$. A somewhat similar driving sequence was considered in disordered spin models in Ref. [64]. However, since the drive described by Eqs. (31) and (32), like that of Ref. [64] and also that of Ref. [66] are not smooth, slow heating and a stretched-exponentially long-lived prethermal plateau is not guaranteed by our results. To circumvent this problem, we replace the sharp peaks in Eq. (32) with smoothed-out approximations, as we discuss in more detail later. 
Let us now observe that $f(\boldsymbol{\theta})$ satisfies $\bar{f}=Q_{j} \omega_{j}$, where $Q=\left(\begin{array}{ll}1 / 2 & 1 / 2\end{array}\right)$. Therefore, we can apply the discussion of Sec. III C and pass to a frame-twisted high-frequency limit. Going into the interaction frame of $H_{0}(\boldsymbol{\theta})$, we can compute the time-quasiperiodic interaction Hamiltonian $H_{\text {int }}(\boldsymbol{\theta})=U_{0}^{\dagger}(\boldsymbol{\theta}) V U_{0}(\boldsymbol{\theta})$. The periodicity of $H_{\text {int }}(\boldsymbol{\theta})$ is on the lattice $\mathcal{L}^{\prime}$, generated by the translation vectors $\tau_{1}=$ $2 \pi(1,1)$ and $\tau_{2}=2 \pi(-1,1)$. Here, $\mathcal{L}^{\prime}$ is a sublattice of the original lattice $\mathcal{L}=2 \pi \mathbb{Z}^{m}$ of symmetry vectors of $H(\boldsymbol{\theta})$. One also sees that $H_{\text {int }}(\boldsymbol{\theta})$ possesses a single nontrivial twisted TTS corresponding to a translation by $\tilde{\boldsymbol{\tau}}=$ $2 \pi(1,0)$ or $\tilde{\boldsymbol{\tau}}=2 \pi(0,1): H_{\text {int }}(\boldsymbol{\theta}+\tilde{\boldsymbol{\tau}})=g H_{\text {int }}(\boldsymbol{\theta}) g^{\dagger}$, with $g=\prod_{i} \sigma_{i}^{x}$. Indeed, the operator $g$ generates the finite group $\mathcal{G} \cong \mathcal{L} / \mathcal{L}^{\prime}=\mathbb{Z}_{2}$.

We next construct the effective time-independent Hamiltonian $D$ from the $H_{\text {int }}$ in a high-frequency expansion, using our approach in Sec. VII. However, for our purpose here of understanding its steady states, it suffices to understand the leading-order Hamiltonian $D^{(0)}$ in the highfrequency expansion: Since $D$ and $D^{(0)}$ are perturbatively close, by construction, the steady states of $D$ and $D^{(0)}$ are in the same universality class. For our expansion in Sec. VII, the leading-order term $D^{(0)}$ is the average of the interaction Hamiltonian

$$
D^{(0)}=\frac{1}{8 \pi^{2}} \int_{0}^{2 \pi} d \theta_{2} \int_{0}^{4 \pi} d \theta_{1} H_{\text {int }}(\boldsymbol{\theta}) .
$$

We note, in particular, the bounds on integration, corresponding to a unit cell of $\mathcal{L}^{\prime}$. We find that, if the driving profile is given by Eqs. (31) and (32), then

$$
D^{(0)}=\sum_{i<j} J_{i j} \sigma_{i}^{z} \sigma_{j}^{z}
$$

More generally, in order to achieve slow heating, we need to smooth out the driving profile, for example, by replacing Eq. (32) with

$$
\Delta_{N}(\theta)=\frac{1}{2 \pi} \sum_{|n|<N}\left(1-\frac{|n|}{N}\right) e^{-2|n| / N} e^{i n(\theta-\pi)}
$$

Here, $\Delta_{N}(\theta)$ is a smooth function that approximates the delta function comb $\Delta(\theta)$ increasingly well as $N \rightarrow \infty$ and is related to the so-called "Fejer kernel." With this replacement, we find

$$
D^{(0)}=\sum_{i<j} J_{i j}\left(a(N) \sigma_{i}^{z} \sigma_{j}^{z}+b(N) \sigma_{i}^{y} \sigma_{j}^{y}\right),
$$

where $a(N), b(N)$ are numerical constants depending on the smoothness parameter $N$. Furthermore, for large $N$, we note that the ratio $a(N) / b(N)$ will be large. For example, if $N=20$, then $a(20)=0.866$ and $b(20)=0.134$.

In accordance with Sec. III B, observe that Eq. (36) is Ising symmetric; that is, $\left[D^{(0)}, \prod_{i} \sigma_{i}^{x}\right]=0$. Furthermore,
$D^{(0)}$ is dominated by Ising interactions $\sigma_{i}^{z} \sigma_{j}^{z}$ along the $z$ direction. Therefore, the steady states of $D$ in the preheating regime are as follows. Provided the initial state has energy density (measured with respect to $D^{(0)}$ ) below some critical energy density, the system will prethermalize to a Gibbs state $\rho_{\beta}$ that spontaneously breaks the Ising symmetry $g=\prod_{i} \sigma_{i}^{x}$. This means that the expectation value of an operator that is odd under the symmetry $g$, such as the local magnetization along the $z$ direction $\sigma_{i}^{z}$, is generically not zero, i.e., $\operatorname{Tr}\left[\sigma_{i}^{z} \rho_{\beta}\right] \neq 0$.

From the discussion in Secs. IV and IV A, we see that the state $\rho(\boldsymbol{\theta})$, Eq. (25), has periodicity on the lattice $\mathcal{L}_{\mathrm{SSB}}=\mathcal{L}^{\prime}$, and the corresponding reciprocal lattice $\mathcal{L}_{\mathrm{SSB}}^{*}$ is generated by the vectors $\boldsymbol{\alpha}_{1}=(1 / 2,1 / 2)$ and $\boldsymbol{\alpha}_{2}=(1 / 2,-1 / 2)$. Thus, the power spectrum of a local observable will generically have peaks at the frequencies

$$
\Omega=\frac{1}{2}\left[n_{1}\left(\omega_{1}-\omega_{2}\right)+n_{2}\left(\omega_{1}+\omega_{2}\right)\right],
$$

where $n_{1}, n_{2} \in \mathbb{Z}$.

Which peaks dominate, however, depends on the operator measured and its symmetry properties under $g$. As a concrete example, suppose we were to measure the local observable $\sigma_{i}^{z}$ (which is odd under $g$ ), whose expectation value in time can be written as

$$
s(t) \approx \operatorname{Tr}\left[\sigma_{i}^{z} \mathcal{V}(t) U_{0}(t) \rho_{\beta} U_{0}(t)^{\dagger} \mathcal{V}(t)^{\dagger}\right] .
$$

Because of the periodicity of $\rho(\boldsymbol{\theta})$, it can be expressed as $s(t)=s\left(\boldsymbol{\omega} t+\boldsymbol{\theta}_{0}\right)$ for an underlying $s(\boldsymbol{\theta})=\operatorname{Tr}\left[\boldsymbol{\sigma}_{i}^{z} \rho(\boldsymbol{\theta})\right]$ that has periodicity in $\mathcal{L}_{\mathrm{SSB}}$, too. To leading order in inverse frequency [i.e., treating $\mathcal{V}(t) \approx \mathbb{I}$ ], the signal can be analytically derived and is given by

$$
s(t) \approx \operatorname{Tr}\left[\sigma_{i}^{z} \rho_{\beta}\right] \cos g(\boldsymbol{\omega} t)+\operatorname{Tr}\left[\sigma_{i}^{y} \rho_{\beta}\right] \sin g(\boldsymbol{\omega} t),
$$

where

$$
\begin{gathered}
g(\boldsymbol{\theta})=\pi\left[\Theta_{N}\left(\theta_{1}\right)+\Theta_{N}\left(\theta_{2}\right)\right], \\
\Theta_{N}(\theta):=\int_{0}^{\theta} d \theta^{\prime} \Delta_{N}\left(\theta^{\prime}\right) .
\end{gathered}
$$

Note that in the case of a delta function driving $(N \rightarrow \infty)$, this signal reduces to

$$
\begin{gathered}
s(t) \approx \operatorname{Tr}\left[\sigma_{i}^{z} \rho_{\beta}\right] \sigma\left(\omega_{1} t\right) \sigma\left(\omega_{2} t\right), \\
\sigma(\theta):=(-1)^{\lceil(\theta-\pi) /(2 \pi)\rceil} .
\end{gathered}
$$

In Figs. 4(b) and 4(d), we plot both $s(t)$ [Eq. (39)] and the underlying function $s(\boldsymbol{\theta})$ from which it is derived, assuming $\operatorname{Tr}\left[\sigma_{i}^{z} \rho_{\beta}\right]=0.8$ and $\operatorname{Tr}\left[\sigma_{i}^{y} \rho_{\beta}\right]=0$ and taking $\omega_{1}=1, \omega_{2}=(1+\sqrt{5}) / 2$ (the golden ratio). Figure $4(\mathrm{e})$ 

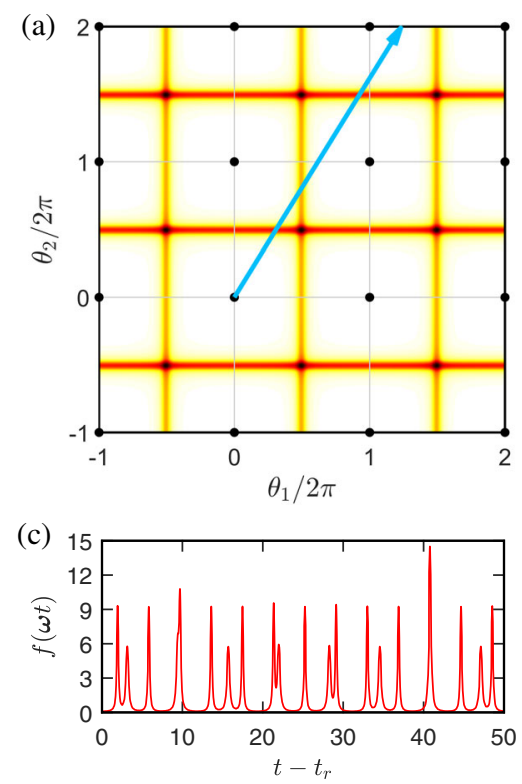
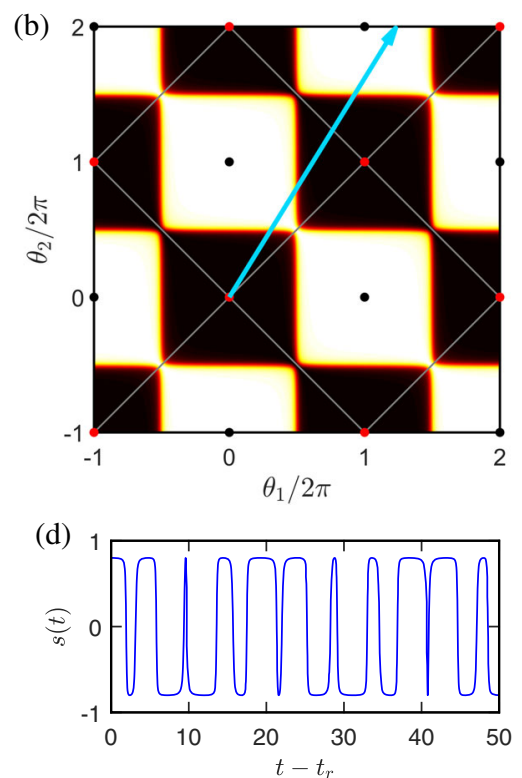

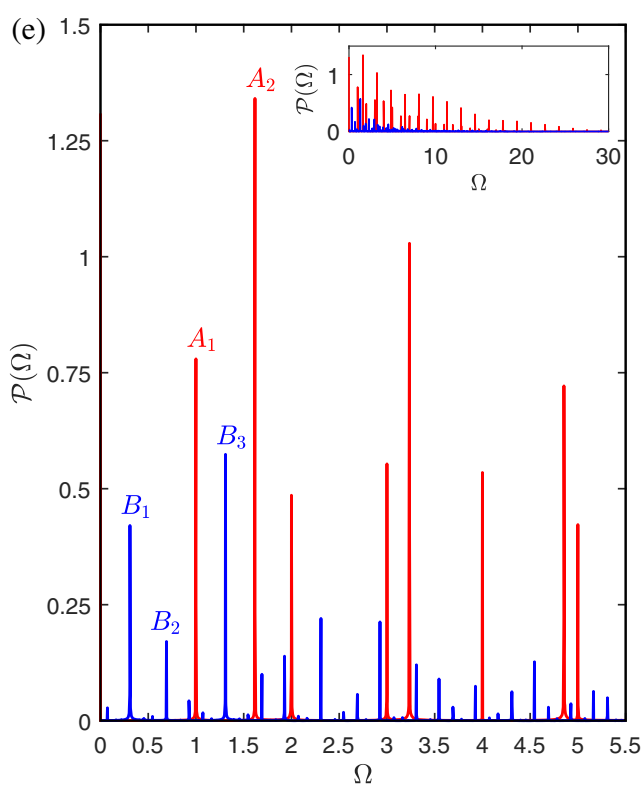

FIG. 4. $\mathbb{Z}_{2}$ DTQC of Sec. IV B: Driving function (a,c) and time-quasicrystal response (b,d). The spontaneous symmetry breaking of time-translation symmetries as seen in the power spectrum (e) of a local observable. (a) Driving function $f(\boldsymbol{\theta})$ in extended $\boldsymbol{\theta}$ space, with $\Delta(\theta)$ in Eq. (31) replaced by $\Delta_{N}(\theta)$ in Eq. (35) with $N=20$. The function is symmetric under translations $\tau \in \mathcal{L}=2 \pi \mathbb{Z}^{2}$ (black points). Grey boxes represent the unit cell that is the standard torus. The blue arrow is the single time trajectory $\boldsymbol{\theta}=\boldsymbol{\omega} t$. (b) In contrast, the observable $s(\boldsymbol{\theta})=\operatorname{Tr}\left[\sigma_{i}^{z} \rho(\boldsymbol{\theta})\right]$ is symmetric under translations by $\boldsymbol{\tau}^{\prime}=2 \pi(1, \pm 1) \mathbb{Z}$, which defines a symmetry-breaking sublattice $\mathcal{L}_{\mathrm{SSB}}=\mathcal{L}^{\prime}$ (red points). The grey boxes represent the unit cell, which is enlarged and rotated by $45^{\circ}$ with respect to the driving Hamiltonian's unit cell. (c) Driving profile $f(t)=f(\omega t)$ as a smooth function in the time domain. Times are measured from the relaxation time $t_{r} \sim J^{-1}$. (d) Discrete time quasicrystal response $s(t)=s(\omega t)$ in the time domain. (e) Power spectra $\mathcal{P}(\Omega)$ of both the driving function $f(t)$ (red) and the observable $s(t)$ (blue). The power spectrum of $f(t)$ exhibits peaks at the base frequencies $\Omega=\boldsymbol{n} \cdot \boldsymbol{\omega}$, with dominant peaks at $\Omega=\omega_{1}\left(A_{1}\right)$ and $\Omega=\omega_{2}\left(A_{2}\right)$. The power spectrum of $s(t)$ has peaks instead at frequencies shifted from the base frequencies by fractional values, reflecting the spontaneous breaking of the time-translation symmetries of the driving Hamiltonian. In particular, the dominant peaks are at $\Omega=\left(\omega_{2}-\omega_{1}\right) / 2\left(B_{1}\right), \Omega=\omega_{2}-\omega_{1}\left(B_{2}\right)$, and $\Omega=\left(\omega_{1}+\omega_{2}\right) / 2\left(B_{3}\right)$. The inset shows the power spectra over a wider range of frequencies.

illustrates the corresponding power spectrum $\mathcal{P}(\Omega)$ of the signal $s(t)$. We see that the spectrum contains peaks at the particular frequencies $\Omega=\left(n_{1}+1 / 2\right) \omega_{1}+\left(n_{2}+1 / 2\right) \omega_{2}$, where $n_{1}, n_{2} \in \mathbb{Z}$; furthermore, those frequencies given by small values of $n_{1}, n_{2}$ contribute the most. These frequencies are a subset of the ones described by Eq. (37) (in particular, they do not include the harmonics of the original driving frequencies, i.e., $\Omega=n_{1} \omega_{1}+n_{2} \omega_{2}$ ), but at higher orders in the inverse frequency, we expect peaks to occur at all values, Eq. (37).

While the $\mathbb{Z}_{2}$ DTQC discussed here is a particularly simple case, we can construct more complicated DTQCs such as ones characterized by the spontaneously broken emergent symmetry groups $\mathcal{G}=\mathbb{Z}_{2} \times \mathbb{Z}_{2}$ (Fig. 5) or $\mathbb{Z}_{3} \times$ $\mathbb{Z}_{2}$ (Fig. 6) in an analogous fashion. We give the explicit form of the systems and drives to realize these DTQCs in Appendix D.

\section{The MBL-DTQC}

We are not restricted to realizing DTQC phases when the Hamiltonian $D$ is thermalizing. Instead, we can consider the case where $D$ exhibits MBL phenomenology and spontaneously breaks the emergent symmetries $g_{\tau}$. Then, the local integrals of motion $\tau_{i}^{z}$ are themselves not invariant under $g_{\tau}$, which is the usual sense of spontaneous symmetry breaking in MBL; see Refs. [74,88-91]. For the model we considered in Eq. (30), we can pick strongly disordered interactions $J_{i j}$, which leads to a "MBL spin glass" effective Hamiltonian with Ising symmetry [88,91]. In the laboratory frame, this set-up gives rise to a MBL DTQC, whose properties we now describe.

As stated in Sec. II D, the defining property of MBL in a quasiperiodically driven system is that there is a complete set of commuting operators $\tilde{\tau}_{i}^{z}$ (the $l$-bits), which evolve quasiperiodically under reverse Heisenberg evolution $\tilde{\tau}_{i}^{z}(t)_{R}=U(t) \tilde{\tau}_{i}^{z} U(t)^{\dagger}$. We can define TTS to be spontaneously broken in a quasiperiodically driven MBL system if the quasiperiodicity of $\tilde{\tau}_{i}^{z}(t)_{R}$ is different from the one of the drive. In other words, writing $\tilde{\tau}_{i}^{z}(t)_{R}=\tilde{\tau}_{i}^{z}\left(\boldsymbol{\omega} t+\boldsymbol{\theta}_{0}\right)$, a TTS corresponding to $\tau \in \mathcal{L}$ is spontaneously broken if $\tilde{\tau}_{i}^{z}(\boldsymbol{\theta}+\boldsymbol{\tau}) \neq \tilde{\tau}_{i}^{z}(\boldsymbol{\theta})$.

For discrete time crystals in periodically driven systems, a key feature is the spectral pairing between eigenstates of the Floquet operator $U_{F}$. For example, in the case 

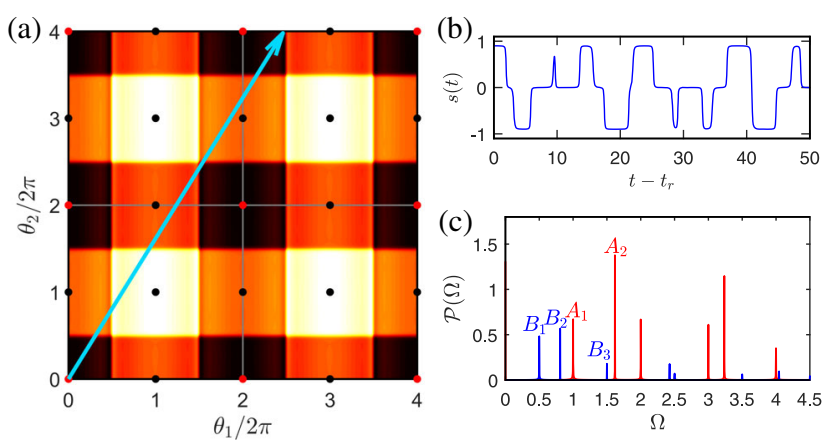

FIG. 5. $\mathbb{Z}_{2} \times \mathbb{Z}_{2}$ DTQC with the model and observables detailed in Appendix D 1. (a) Observable $s(\boldsymbol{\theta})$ in $\boldsymbol{\theta}$ space at leading order in inverse frequency, displaying the symmetry-breaking pattern lattice $\mathcal{L}_{\mathrm{SSB}}$ (red points) as well as the original lattice of translations $\mathcal{L}$ (black points). Grey boxes denote the symmetrybreaking unit cell. (b) Discrete time quasicrystal response $s(t)=s(\omega t)$. (c) Power spectra $\mathcal{P}(\Omega)$ of both the driving function (red) and the observable $s(t)$ (blue). Dominant peaks in the driving function correspond to $\Omega=\omega_{1}\left(A_{1}\right)$ and $\Omega=\omega_{2}\left(A_{2}\right)$. Dominant peaks of the observable $s(t)$ correspond to $\Omega=\omega_{1} / 2$ $\left(B_{1}\right), \Omega=\omega_{2} / 2\left(B_{2}\right), \Omega=3 \omega_{1} / 2\left(B_{3}\right)$. Going beyond leading order in inverse frequency, an observable's power spectrum can exhibit peaks at the frequencies $\Omega=\frac{1}{2} \boldsymbol{n} \cdot \boldsymbol{\omega}$ for $\boldsymbol{n} \in \mathbb{Z}^{2}$.

corresponding to a discrete time crystal with period doubling, these eigenstates always come in cat state pairs separated in quasienergy by $\pi$. However, generalizing this concept to DTQC is subtle because of a lack of a single time-evolution operator like $U_{F}$.

Finally, let us mention what behavior is expected for measurable observables for a MBL DTQC. We know that
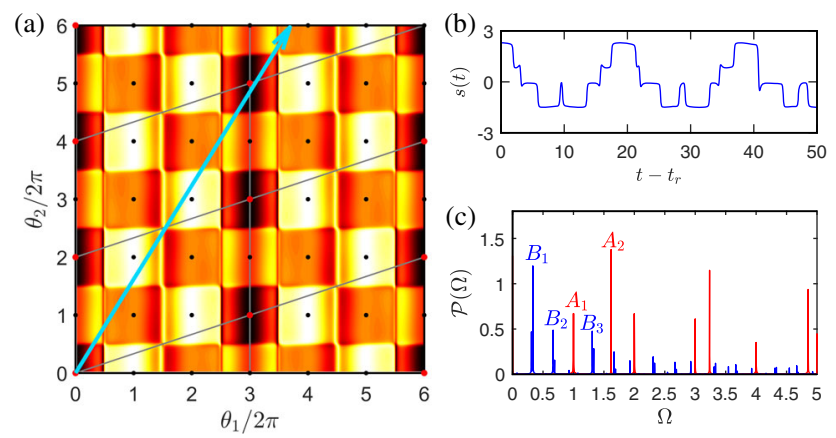

FIG. 6. $\quad \mathbb{Z}_{3} \times \mathbb{Z}_{2}$ DTQC with the model and observable detailed in Appendix D 2. (a) Observable $s(\boldsymbol{\theta})$ in $\boldsymbol{\theta}$ space at leading order in inverse frequency, displaying the symmetry-breaking pattern lattice $\mathcal{L}_{\mathrm{SSB}}$ (red points) as well as the original lattice of translations $\mathcal{L}$ (black points). Grey boxes denote the symmetry-breaking unit cell. (b) Discrete time quasicrystal response $s(t)=s(\boldsymbol{\omega} t)$. (c) Power spectra $\mathcal{P}(\Omega)$ of both the driving function (red) and the observable $s(t)$ (blue). Dominant peaks in the driving function correspond to $\Omega=\omega_{1}\left(A_{1}\right)$ and $\Omega=\omega_{2}\left(A_{2}\right)$. Dominant peaks of the observable $s(t)$ correspond to $\Omega=\omega_{1} / 3$ $\left(B_{1}\right), \Omega=2 \omega_{1} / 3\left(B_{2}\right), \Omega=\left(\omega_{1}+\omega_{2}\right) / 2\left(B_{3}\right)$. Going beyond leading order in inverse frequency, an observable's power spectrum can exhibit peaks at frequencies $\Omega=n_{1}\left(\omega_{1} / 3\right)+$ $n_{2}\left[-\left(\omega_{1} / 6\right)+\left(\omega_{2} / 2\right)\right]$ for $n_{1}, n_{2} \in \mathbb{Z}$. for MBL systems, local observables relax to a steady state, generically as a power law in time [92]. Once this steady state is achieved, the power spectrum of the time dependence of local observables will display the same behavior as discussed in the prethermal case, by the same arguments. At shorter times before the steady state is achieved, one expects analogous behavior to what is seen for the discrete time crystal in Floquet systems: In addition to "universal" peaks in the power spectra of observables that persist to infinite times, one also sees other nonuniversal peaks that are dependent on the precise disorder realization of the system.

\section{How sharply distinct is the DTQC phase?}

In this subsection, we wish to elaborate on a point we made earlier regarding the DTQC phase. The DTQC clearly looks like a spontaneous symmetry-breaking phase in terms of the extended space picture, as seen, for example, in Figs. 4(b), 5(a), and 6(a). However, the extended space picture is a purely formal construction, and what we actually measure are observables as a function of a single time. Here, we wish to be precise about the sense in which the DTQC is distinct from the trivial phase in terms of the single time. We also discuss the impact of the fact that we can only observe the system over a finite time window because of both practical experimental limitations and the finiteness of the heating time $t_{*}$.

The main point, as we have seen, is that the sharp order parameter for the DTQC is the existence of "subharmonic" peaks, i.e., the nonzero amplitude of a peak in the power spectrum at frequency $\Omega_{\boldsymbol{\alpha}}=\boldsymbol{\alpha} \cdot \boldsymbol{\omega}$, where $\boldsymbol{\alpha} \in \mathcal{L}_{\mathrm{SSB}}^{*}$, which is not simply a harmonic of the applied frequencies; that is, $\Omega_{\alpha} \neq \boldsymbol{n} \cdot \boldsymbol{\omega}$ for any integer vector $\boldsymbol{n}$. This statement is meaningful even though, for a quasiperiodic drive, the harmonics $\Omega_{n}$ are dense. To see this, consider, for example, the DTQC described in Sec. IV B, which has a peak at frequency $\Omega_{\alpha}$ for $\boldsymbol{\alpha}=(1 / 2,1 / 2)$. If $\Omega_{\alpha}=\Omega_{n}$ for some $\boldsymbol{n}$, then this would imply that $(\boldsymbol{n}-\boldsymbol{\alpha}) \cdot \boldsymbol{\omega}=0$, and hence that $\omega_{1} / \omega_{2}=\left(n_{2}-1 / 2\right) /\left(n_{1}-1 / 2\right)$, which is a rational number, contradicting our assumption that $\omega_{1} / \omega_{2}$ is irrational.

Of course, the above argument presupposes that we have infinitely good frequency resolution, corresponding to observing the system over an infinitely long time. Let us instead consider the case where we have some finite frequency resolution $\delta$, corresponding to a finite observation time $\tau \sim 1 / \delta$. Suppose that $\left|\Omega_{\alpha}-\Omega_{n}\right|<\delta$ for some $\Omega_{\alpha}$ not expressible as an integer harmonic of the applied frequencies. We estimate how large $|\boldsymbol{n}|$ has to be in order for this condition to hold. To achieve this estimate, we assume the frequency vector $\boldsymbol{\omega}$ obeys a so-called Diophantine condition, which we introduce in Sec. VI; see Eq. (55), which quantifies precisely how small $\boldsymbol{n} \cdot \boldsymbol{\omega}$ can be as a function of $|\boldsymbol{n}|$. Applying this condition with $\gamma=$ $m-1+\epsilon$ [and replacing, for example, $\boldsymbol{n} \rightarrow 2 \boldsymbol{\alpha}-2 \boldsymbol{n}$ if $\boldsymbol{\alpha}=(1 / 2,1 / 2)]$, we find that 


$$
|\boldsymbol{n}| \geq C(\delta /|\boldsymbol{\omega}|)^{-1 /(m-1+\epsilon)}
$$

for some constant $C$ that does not depend on $\delta$ or on the overall frequency scale. Since the amplitude of peaks decays exponentially with $\boldsymbol{n}$ due to the smoothness of the drive, we see that, as we increase our frequency resolution, the amplitude of harmonic peaks that would not be resolvable from the subharmonic DTQC peak goes to zero stretched exponentially fast. In other words, if we fix the highest-order $n_{\max }$ of harmonic peaks that we want to consider, then the resolution $\delta$ required to distinguish the DTQC phase from the trivial phase is determined by imposing

$$
C(\delta /|\boldsymbol{\omega}|)^{-1 /(m-1+\epsilon)} \geq n_{\max } .
$$

Equivalently, the observation time $\tau$ has to satisfy

$$
\tau \geq|\boldsymbol{\omega}|^{-1}\left(n_{\max } / C^{\prime}\right)^{m-1+\epsilon}
$$

for some numerical constant $C^{\prime}$. Formally, the phases are sharply distinct only when $n_{\max } \rightarrow \infty$, which requires $\delta \rightarrow 0$. However, the phases are more or less distinct "in practice" provided Eq. (45) or Eq. (46) is satisfied for some sufficiently large $n_{\max }$. This statement is similar to the familiar idea that phases of matter are formally sharply distinct only in the thermodynamic limit but, in practice, are distinct as long as the system size is much larger than the correlation length. Note that the scalings of Eqs. (45) and (46) get worse as $m$ becomes large; that is, as the number of incommensurate frequencies grows, we need to observe the system over very long times in order to distinguish phases.

Let us observe that Eq. (45) has a very appealing physical interpretation [93] in terms of the picture of quasiperiodic driving where time sweeps out a path in the phase torus, as shown in Fig. 1(b). After time $\tau$ has elapsed, the proportion of the phase torus that is within distance $\Delta$ of the orbit scales like $\sim \Delta^{m-1}|\omega|^{\tau}$. Therefore, if we identify the frequency resolution $\delta$ with the reciprocal of the time $\tau$ over which we observe the system, then Eq. (45) (if we neglect $\epsilon$ ) is the condition for the fraction of the torus within distance $1 / n_{\max }$ of the orbit to be $\sim 1$. This condition makes sense because, in order for different quasiperiodic phases to be distinct, the system needs to "know" that it is quasiperiodic; that is, it needs to explore the phase torus sufficiently densely.

Finally, let us recall that the heating time $t_{*}$ sets an upper bound on the observation time $\tau$. Thus, the phases are formally sharply distinct only in the infinite frequency limit where $t_{*} \rightarrow \infty$. Nevertheless, since $t_{*}$ grows stretched exponentially fast with frequency, we can always increase the frequency while keeping all the other parameters of the problem fixed, in order to have $\tau<t_{*}$ while still satisfying Eqs. (45) and (46) for $n_{\max } \gg 1$, and so that the phases are distinguishable in practice.

\section{QUASIPERIODIC TOPOLOGICAL PHASES}

\section{A. Eigenstate classification of quasiperiodic topological phases}

In addition to spontaneous symmetry-breaking phases like the DTQC, one can also consider topological phases protected by multiple TTSs. In discussing these topological phases, we focus on situations where the driving Hamiltonian has sufficiently strong disorder, so the preheating Hamiltonian $D$ is MBL (see Sec. II D). We remark here that it is not completely settled whether MBL for spatial dimensions greater than one can exist in the strict sense (that is, as an infinite-time phenomenon), with some arguments suggesting that, at least for uncorrelated disordered local potentials, it will be destabilized by an "avalanche" mechanism [94-97]. However, such a mechanism is highly suppressed for sufficiently strong disorder (compared to other local energy scales of the system), so it is expected that there is a long timescale $t_{\text {avalanche }}$ [97] up to which the effect of avalanches can be neglected and the system exhibits MBL phenomenology. Indeed, such localizing behavior has been verified experimentally in disordered systems in two spatial dimensions $[98,99]$. In our present case, as the heating time $t_{*}$ sets a fundamental limit on the physics we describe anyway, we only require that MBL is sufficiently long-lived, in the sense that $t_{\text {avalanche }}$ is comparable to or greater than $t_{*}$, which can be straightforwardly achieved with appropriate driving and system parameters.

Since MBL eigenstates have properties analogous to the ground state of gapped local Hamiltonians at all energy densities [100], the topological classification of such ground states can be applied to each state of $D$. Static MBL phases can therefore be distinguished by the topology of their eigenstates; this is referred to as eigenstate order $[88,101,102]$. In driven systems, the concept of eigenstate order is enriched [69,103-107] as there are additional topological features arising from the time evolution. Consider the Floquet time-evolution operator, Eq. (4), in the preheating regime, where we temporarily treat the decomposition as exact. For an eigenstate $|\Psi\rangle$ of $D$ with energy eigenvalue $\epsilon$, define a time evolution

$$
|\Psi(t)\rangle=e^{i \epsilon t} U(t) P(0)|\Psi\rangle=P(t)|\Psi\rangle .
$$

We refer to this time evolution as the micromotion of the eigenstate $|\Psi\rangle$. Even when the eigenstate $|\Psi\rangle$ itself describes a topologically trivial static phase, its corresponding micromotion $|\Psi(t)\rangle$ could still be nontrivial. More precisely, let $\Omega_{d}$ denote the space of all possible gapped ground states of quasilocal Hamiltonians in $d$ spatial dimensions (we mod out by global phase factors in the definition of points in $\Omega_{d}$ ). In Floquet systems, the micromotion is periodic and defines a loop in $\Omega_{d}$-we say the micromotion is nontrivial if this loop is not contractible to a point. 
We can generalize the notion of topological micromotion, Eq. (47), to the case of quasiperiodically driven systems, using the decomposition in Eq. (6). The micromotion $|\Psi(t)\rangle=P(t)|\Psi\rangle$ is now quasiperiodic and can be expressed as $|\Psi(t)\rangle=\left|\Psi\left(\boldsymbol{\omega} t+\boldsymbol{\theta}_{0}\right)\right\rangle$, where $|\Psi(\boldsymbol{\theta})\rangle$ is parametrized by the torus $\mathbb{T}^{m}$. This evolution now defines a map on $\mathbb{T}^{m} \rightarrow \Omega_{d}$. If this map cannot be continuously deformed to the constant map, the evolution is nontrivial.

The question of how to classify maps $\mathbb{T}^{m} \rightarrow \Omega_{d}$, or more generally maps $\mathcal{X} \rightarrow \Omega_{d}$ for any space $\mathcal{X}$, remains, in principle, an open problem. However, there are good reasons [108-115] to conjecture that the answer to this question is already contained within the frameworks used to classify stationary topological phases. See Appendix E for a more technical discussion.

Here, we focus on a special class of phases, which are natural generalizations of bosonic SPT phases and already display a rich set of behaviors. Recall that equilibrium bosonic SPT phases with unitary symmetry $G$ in $d$ spatial dimensions are believed to be partially classified ${ }^{1}$ by the group cohomology $\mathcal{H}^{d+2}(G, \mathbb{Z})$ [116-119]. We can also write group cohomology as the singular cohomology of the so-called classifying space $B G$ of the group $G: \mathcal{H}^{d+2}(G, \mathbb{Z}) \cong$ $H^{d+2}(B G, \mathbb{Z})$. The idea is that maps $\mathcal{X} \rightarrow \Omega_{d}$ in the presence of symmetry $G$ should be partially classified by replacing $B G \rightarrow \mathcal{X} \times B G$; i.e., the classification is $H^{d+2}(\mathcal{X} \times B G, \mathbb{Z})$. In fact, under general conditions for both SPT and SET phases of bosons or fermions, the classification can be derived from the classification of equilibrium SPT and SET phases simply by replacing $B G \rightarrow \mathcal{X} \times B G$. We give some justification for this in Appendix E.

Using the fact that $B \mathbb{Z}^{m}=\mathbb{T}^{m}$, we get the following powerful result. We find that the classification of maps $\mathbb{T}^{m} \rightarrow \Omega_{d}$ in the presence of symmetry $G$ is in one-toone correspondence with the classification of stationary symmetry-protected and symmetry-enriched phases with symmetry $\mathbb{Z}^{m} \times G$. The interpretation of the additional $\mathbb{Z}^{m}$ symmetry is that it corresponds to the multiple timetranslation symmetries referred to in Sec. III B. We call this statement of equivalence of classifications the "quasiperiodic equivalence principle." The periodic case, which we could call the "Floquet equivalence principle," was discussed in Refs. [69,107]; compare also the "crystalline equivalence principle" of Ref. [113].

The simplest cases of fundamentally nonequilibrium topological phases are ones where eigenstates $|\Psi\rangle$ are themselves in the trivial $G$ SPT phase. In this case, the classification of maps $\mathbb{T}^{m} \rightarrow \Omega_{d}$ with a $G$ symmetry imposed has the general decomposition

\footnotetext{
${ }^{1}$ Note that this classification is often stated as $\mathcal{H}^{d+1}(G, \mathrm{U}(1))$, which is equivalent for compact groups provided that the cohomology with U(1) coefficients is defined appropriately. However, here we are dealing with noncompact groups such as translations and must use $\mathcal{H}^{d+2}(G, \mathbb{Z})$.
}

$$
\bigoplus_{r=1}^{m} \bigoplus_{\left(k_{1} \cdots k_{r}\right)} \mathcal{C}_{d-r}
$$

where $\bigoplus_{\left(k_{1} \cdots k_{r}\right)}$ indicates a sum over all possible choice of nonrepeating numbers $k_{1}, \ldots, k_{r} \in\{1, \ldots, m\}$. Here, $\mathcal{C}_{s}$ is the classification of equilibrium SPT phases or invertible topological orders with $G$ symmetry in $s$ spatial dimensions for $s \geq 0$; for $s<0$, we set $\mathcal{C}_{s}=\pi_{-s}\left(\Omega_{0}\right)$. For the case of the group cohomology classification $\mathcal{H}^{d+2}\left(\mathbb{Z}^{\times m} \times G, \mathbb{Z}\right)$, Eq. (48) can be proven using the Künneth formula [120], although the result holds more generally (see Appendix E).

Equation (48) has a simple physical interpretation: The different terms correspond to cases where $r$ time-translation symmetries are "essentially" involved in the definition of the corresponding phases. One term corresponds to maps $\mathbb{T}^{m} \rightarrow \Omega_{d}$ that depend on all $m$ incommensurate frequencies, which are classified by $\mathcal{C}_{d-m}$. The remaining terms of Eq. (48) depend only on $r<m$ frequencies, corresponding to micromotions that vary on $r$-dimensional hyperplanes $\mathbb{T}^{r} \subseteq \mathbb{T}^{m}$ and are deformable to constant evolutions in the other directions.

For periodic drives $(m=1)$, Eq. (48) reduces to just $\mathcal{C}_{d-1}$. In this case, we can think of the nontriviality of the micromotion as a pump per Floquet cycle, which nucleates equilibrium $(d-1)$-dimensional SPT phases and transports them onto the boundary of the system [69,103,104,121]. For $m \geq 2, \mathcal{C}_{d-m}$ terms in Eq. (48) can be interpreted as a higher-order pump ("pump of pumps"). However, making this notion concrete for observables on the boundary of model systems is beyond the current discussion.

Let us now focus on the micromotions that depend on all $m$ frequencies. The ideas of Sec. III allow us to construct nontrivial micromotions in a preheating regime through what we refer to as a "bootstrap construction." Indeed, suppose that we choose $Q=(1 / N) \mathbb{I}_{m}$ for integer $N$, so that $\mathcal{G}=\mathbb{Z}_{N}^{\times m}$. For concreteness, we consider the case $m=2$. Then, the effective Hamiltonian $D$ has an enhanced symmetry $G \times \mathcal{G}$, and accordingly, it can host bosonic SPT phases protected by $G \times \mathcal{G}$, which are classified by $\mathcal{H}^{d+2}(G \times \mathcal{G}, \mathbb{Z})$. Invoking the Künneth formula, we see that this term contains a factor

$$
\mathcal{H}^{1}\left(\mathbb{Z}_{N}, \mathcal{H}^{1}\left(\mathbb{Z}_{N}, \mathcal{H}^{d}(G, \mathbb{Z})\right)\right)
$$

Suppose we ensure that the effective Hamiltonian $D$ has eigenstates that are in a SPT phase associated with the emergent $\mathcal{G} \times G$ symmetry corresponding to an element of Eq. (49). The projection map $\mathbb{Z} \rightarrow \mathbb{Z}_{N}$ induces a map in group cohomology,

$$
\begin{aligned}
\mathcal{H}^{1} & \left(\mathbb{Z}_{N}, \mathcal{H}^{1}\left(\mathbb{Z}_{N}, \mathcal{H}^{d}(G, \mathbb{Z})\right)\right) \\
& \rightarrow \mathcal{H}^{1}\left(\mathbb{Z}, \mathcal{H}^{1}\left(\mathbb{Z}, \mathcal{H}^{d}(G, \mathbb{Z})\right)\right) \\
& \cong \mathcal{H}^{d}(G, \mathbb{Z}),
\end{aligned}
$$


(a)

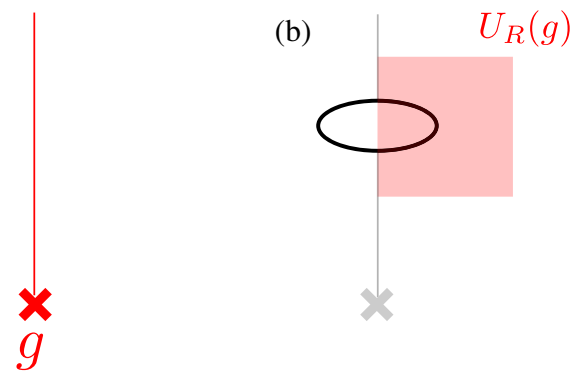

FIG. 7. (a) A symmetry flux for a symmetry $g$ consists of a line terminating at a point. (b) Terms of the Hamiltonian (shown as an oval) that cross the line get conjugated by the unitary action, restricted to act only one side of the line [denoted by $U_{R}(g)$ ].

where we have used the fact that $\mathcal{H}^{1}(\mathbb{Z}, A) \cong A$ for any finite Abelian group $A$. Note that $\mathcal{H}^{d}(G, \mathbb{Z})$ obtained in Eq. (50) corresponds to the classification of SPT phases in $d-2$ dimensions, i.e., $\mathcal{C}_{d-2}$ in the above notation. If the image of the element of Eq. (49) in Eq. (50) is nontrivial, the micromotions corresponding to the eigenstates of $D$ are nontrivial in a way that relies on both frequencies.

\section{B. A minimal example of a nontrivial quasiperiodic topological phase}

In this subsection, we will make more concrete the general and somewhat abstract considerations above. We consider a particular example of a new quasiperiodic topological phase, which cannot occur in either a stationary or in a periodically driven system.

The phase occurs in a two-dimensional spin system driven quasiperiodically with $m=2$ independent frequencies and containing a microscopic symmetry $G=\mathbb{Z}_{2}$. According to the general classification discussed above, there is a $\mathcal{C}_{0}=\mathcal{H}^{2}\left(\mathbb{Z}_{2}, \mathbb{Z}\right)=\mathcal{H}^{1}\left(\mathbb{Z}_{2}, \mathrm{U}(1)\right)=\mathbb{Z}_{2}$ classification of quasiperiodic topological phases that rely on both frequencies. Thus, there is a single nontrivial topological phase in this classification. Below, we give an explicit Hamiltonian construction to realize this phase, but first, we discuss its universal properties.

Following the general approach of this paper, we can make this phase well defined in the twisted high-frequency limit. There, we obtain an effective static Hamiltonian with an emergent $\mathbb{Z}_{2} \times \mathbb{Z}_{2}$ symmetry (coming from the multiple time-translation symmetries) in addition to the microscopic $\mathbb{Z}_{2}$. For the phase to be topological and stable, the Hamiltonian must realize a MBL phase in the bulk whose eigenstates are SPT states under the overall $\mathbb{Z}_{2}^{\times 3}$ symmetry. Such SPT states, however, cannot remain MBL at the boundary while preserving all three $\mathbb{Z}_{2}$ symmetries. Therefore, a dramatic signature of this topological phase, as with SPT phases more generally $[9,122-124]$, is the existence of a nontrivial boundary. Depending on the exact nature of the Hamiltonian on the boundary, either there is a boundary DTQC (which occurs when the emergent $\mathbb{Z}_{2} \times \mathbb{Z}_{2}$ is spontaneously broken), or the microscopic $\mathbb{Z}_{2}$ symmetry is spontaneously broken on the boundary, or there is topologically induced boundary delocalization.

Let us discuss another diagnostic of the nontrivial topology. This diagnostic relates to a "symmetry twist defect" in the bulk, which is a standard probe for SPT phases [125]. We define such a defect by introducing a line terminating at a point (Fig. 7) and conjugating the local terms of the Hamiltonian that straddle the line by the microscopic $\mathbb{Z}_{2}$ symmetry, restricted to act only on one-half of the line. In the nontrivial SPT-MBL phase we are discussing, the Hilbert space of local states near the termination point carries a projective representation of the emergent $\mathbb{Z}_{2} \times \mathbb{Z}_{2}$ symmetry. From this property, combined with the fact that the effective static Hamiltonian $D$ must commute with the emergent $\mathbb{Z}_{2} \times \mathbb{Z}_{2}$, we can deduce the existence of a topologically protected qubit; that is, there exist localized operators $\tau^{z}$ and $\tau^{x}$ near the defect such that $\tau_{z}^{2}=\tau_{x}^{2}=1$, $\tau_{z} \tau_{x}=-\tau_{x} \tau_{z}$, and $\left[\tau^{x}, D\right]=\left[\tau^{z}, D\right]=0$. In other words, there is an effective qubit degree of freedom that does not couple to the rest of the system under time evolution in the rotating frame. In the lab frame, this effective qubit degree of freedom becomes time dependent due to the rotating-frame transformation, but it comes back to arbitrarily close even after very long times due to the recurrences of the quasiperiodic-in-time rotating-frame transformation. Note that this condition is stronger than that from just MBL. In MBL, there are $l$-bit operators $\tau_{i}^{z}$ that preserve the memory of the initial state forever, but normally, their conjugate operators $\tau_{i}^{x}$ would decohere, unlike what happens here. We can imagine probing this effect numerically if we assume that $\tau^{z}$ and $\tau^{x}$ have some nonzero overlap with some local spin operators $\sigma_{i}^{z}$ and $\sigma_{i}^{x}$. Then, the unequal time correlator $\left\langle\sigma_{i}^{\alpha}(t) \sigma_{i}^{\alpha}\right\rangle, \alpha=x, z$, will fail to decay to zero as $t \rightarrow \infty$. Finally, we note that we will find similar behavior if, instead of considering a symmetry twist defect, we introduce a boundary for the system, spontaneously break the microscopic $\mathbb{Z}_{2}$ symmetry on the boundary, and then examine the properties of a domain wall.

It is important to consider to what extent the signatures discussed here depend on the presence of an emergent $\mathbb{Z}_{2} \times \mathbb{Z}_{2}$ symmetry. We know that such an emergent symmetry is always present when we stabilize the phase under consideration through the twisted high-frequency limit, which is the main focus of this paper. Nevertheless, one can imagine, as we have alluded to previously, that, in the presence of strong disorder leading to MBL, quasiperiodically driven phases of matter can be stabilized even in a regime not captured by the twisted high-frequency limit. In such a regime, the concept of eigenstate micromotions giving rise to a phase classification, as discussed in generality in the previous subsection, will still apply, but it is not clear if we still expect an emergent $\mathbb{Z}_{2} \times \mathbb{Z}_{2}$ symmetry to be present. On the other hand, the general 
discussion of the previous subsection shows that with respect to the classification of phases, we can still treat the system as having a $\mathbb{Z} \times \mathbb{Z}$ symmetry.

One can check that the phase we are discussing, which is originally a $\mathbb{Z}_{2} \times \mathbb{Z}_{2} \times \mathbb{Z}_{2}$ SPT (i.e., protected by a combination of the emergent $\mathbb{Z}_{2} \times \mathbb{Z}_{2}$ and the microscopic $\mathbb{Z}_{2}$ symmetry), remains nontrivial if we relax the symmetry group to $\mathbb{Z} \times \mathbb{Z} \times \mathbb{Z}_{2}$. What may be somewhat in doubt, however, is whether the topologically protected qubit associated with the symmetry twist, as discussed above, remains robust, for reasons that we discuss in more detail in Appendix F. However, the property of the boundary not being localizable while preserving the microscopic $\mathbb{Z}_{2}$ symmetry and the quasiperiodicity of the drive should still hold. Moreover, in Appendix F, we also argue that a more robust feature of the symmetry twist defect should be that it hosts a "topological pump" of energy between the two incommensurate frequencies (see Ref. [59] and also Sec. V C) that is half-quantized.

\section{Example Hamiltonian}

Here, we give an explicit construction of a Hamiltonian on the lattice, realizing the phase discussed above. Consider a square lattice in two spatial dimensions with spin- $1 / 2$ particles on the links $(A)$, on the sites $(B)$, and on the plaquettes $(C)$, as shown in Fig. 8. Assume that the system has a microscopic $\mathbb{Z}_{2}$ symmetry generated by $X^{A}:=\prod_{l \in A} \sigma_{l}^{x}$, where the product is over spins on the links. We then define $S_{B}=\frac{1}{2} \sum_{v \in B}\left(\sigma_{v}^{x}+1\right)$, where the sum is over all spins on the vertices, and $S_{C}=\frac{1}{2} \sum_{p \in C}\left(\sigma_{p}^{x}+1\right)$, where the sum is over spins on the plaquettes. Furthermore, $X^{B}=\exp \left(-i \pi S^{B}\right)=$ $\prod_{v \in B} \sigma_{v}^{x}$ and $X^{C}=\exp \left(-i \pi S^{C}\right)=\prod_{p \in C} \sigma_{p}^{x}$. We consider the time-quasiperiodic Hamiltonian

$$
H(t)=f_{1}(t) S_{B}+f_{2}(t) S_{C}+D_{0}+V(t) .
$$

Here, $D_{0}$ is a time-independent Hamiltonian that commutes with $X^{A}, X^{B}$, and $X^{C}$ that we specify explicitly below, and $V(t)$ is some generic perturbation that must commute with the microscopic symmetry $X^{A}$. All the time-dependent quantities $f_{1}(t), f_{2}(t)$, and $V(t)$ are quasiperiodic with frequency vector $\boldsymbol{\omega}=\left(\omega_{1}, \omega_{2}\right)$, assumed to be large compared to the local energy scales of $D_{0}, V(t)$.

We now choose the driving functions $f_{1}(t), f_{2}(t)$ to be

$$
f_{i}(t)=\pi \omega_{i} \Delta_{N}\left(\omega_{i} t\right)
$$

for $i=1,2$. Here, $\Delta_{N}(\theta)$ is the smooth approximation to the Dirac delta comb, as utilized in the DTQC example of Sec. IV B, given in Eq. (35). The drive corresponds to one in which $Q=(1 / 2) \mathbb{I}_{2}$, following the discussion of the frame-twisted high-frequency limit of Sec. III C. This choice of driving function strikes a balance between having a smooth drive, which is necessary to achieve a long-lived

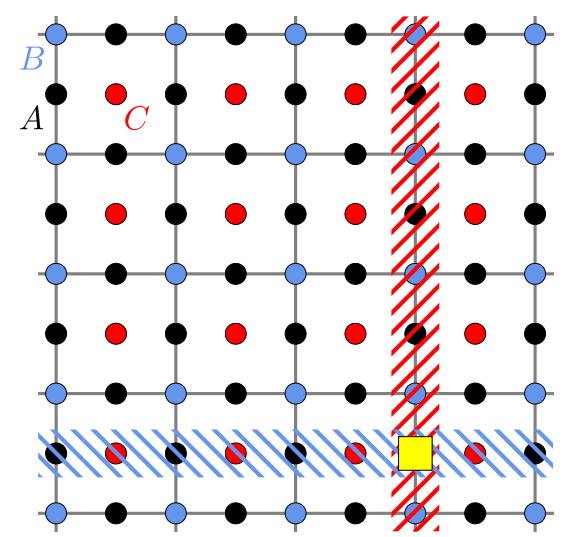

FIG. 8. Lattice of spins used in the construction of a minimal Hamiltonian displaying the quasiperiodic symmetry-protected topological phase introduced in Sec. V B 1. Spins are placed on the links ( $A$, black), vertices ( $B$, blue), and plaquettes $(C$, red) of a $2 \mathrm{D}$ square lattice. The ground state is a superposition of fluctuating domain walls of Ising orders of the $B$ spins (blue hatched region) and $C$ spins (red hatched region). In the topological phase, the intersection of these domain wall (yellow square) binds an Ising charge of $A$.

preheating regime, and the property that the interaction Hamiltonian $\quad H_{\text {int }}(\boldsymbol{\theta})=U_{0}(\boldsymbol{\theta})^{\dagger}(D+V(\boldsymbol{\theta})) U_{0}(\boldsymbol{\theta})$ (see Sec. IIIC) has the term $U_{0}(\boldsymbol{\theta})^{\dagger} D_{0} U_{0}(\boldsymbol{\theta})$ satisfying, roughly, $U_{0}(\boldsymbol{\theta})^{\dagger} D_{0} U_{0}(\boldsymbol{\theta}) \approx D_{0}$. Accordingly, upon taking the frame-twisted high-frequency limit, we find that there is an effective Hamiltonian $D=D_{0}+\delta D$ in a long-lived preheating regime at high driving frequencies, where $\delta D$ commutes with $X^{A}, X^{B}$, and $X^{C}$, and where the local strength of $\delta D$ is on the order of that of $V(t)$.

Suppose $D_{0}$ is a MBL Hamiltonian whose eigenstates are in the SPT phase for symmetry $\mathbb{Z}_{2}^{\times 3}$ corresponding to the nontrivial element of $\mathbb{Z}_{2} \cong \mathcal{H}^{1}\left\{\mathbb{Z}_{2}, \mathcal{H}^{1}\left[\mathbb{Z}_{2}, \mathcal{H}^{2}\left(\mathbb{Z}_{2}, \mathbb{Z}\right)\right]\right\} \leq$ $\mathcal{H}^{4}\left(\mathbb{Z}_{2}^{\times 3}, \mathbb{Z}\right)$. Indeed, such Hamiltonians can be realized via a decorated domain-wall construction [126], where the ground state is a superposition of fluctuating domain walls. The intersections of $X^{B}$ domain walls and $X^{C}$ domain walls (which precisely occur at $A$ spins) carry a -1 charge of $X^{A}$; see Fig. 8. Concretely, we can write $D_{0}=$ $\mathcal{U}\left(\sum_{v} h_{v} \sigma_{x}^{v}+\sum_{p} h_{p} \sigma_{x}^{p}+\sum_{l} h_{l} \sigma_{x}^{l}\right) \mathcal{U}^{\dagger}$, where the coefficients $h_{v}, h_{l}, h_{p}$ are chosen from some random distribution, and

$$
\begin{aligned}
\mathcal{U}= & \sum_{\substack{\left\{\sigma_{v}\right\},\left\{\sigma_{p}\right\},\left\{\sigma_{l}\right\}= \pm 1}} \prod_{l \in A}(-1)^{\frac{1}{8}\left(1-\sigma_{p_{1}(l)} \sigma_{p_{2}(l)}\right)\left(1-\sigma_{v_{1}(l)} \sigma_{v_{2}(l)}\right)\left(1-\sigma_{l}\right)} \\
& \times\left|\left\{\sigma_{v}\right\},\left\{\sigma_{p}\right\},\left\{\sigma_{l}\right\}\right\rangle\left\langle\left\{\sigma_{v}\right\},\left\{\sigma_{p}\right\},\left\{\sigma_{l}\right\}\right|
\end{aligned}
$$

where $p_{1}(l), p_{2}(l)$ are the two plaquettes adjacent to the link $l ; v_{1}(l), v_{2}(l)$ are the two vertices connected by the link $l$; and $\left|\left\{\sigma_{v}\right\},\left\{\sigma_{p}\right\},\left\{\sigma_{l}\right\}\right\rangle$ is a basis state labeled by the eigenvalues of $\sigma_{v}^{z}, \sigma_{p}^{z}, \sigma_{l}^{v}$ for all vertices $v$, plaquettes $p$, and 
links $l$. Note that if the symmetric term $\delta D$ is sufficiently weak, the effective Hamiltonian $D$ eigenstates will also belong to the same SPT phase as the MBL Hamiltonian $D_{0}$.

Following the general arguments from before, one finds that the eigenstates of $D$ indeed undergo nontrivial micromotion, classified by the nontrivial element of $\mathcal{H}^{1}\left(\mathbb{Z}_{2}, \mathrm{U}(1)\right)=\mathbb{Z}_{2}$, which also classifies SPT phases with $\mathbb{Z}_{2}$ symmetry in zero spatial dimensions (i.e., $\mathbb{Z}_{2}$ charges).

\section{Relation to previous works}

Some aspects of topology in quasiperiodically driven systems have previously been studied, primarily in the context of noninteracting systems. We now briefly mention how these phenomena are related to our classification and construction.

Reference [59] discussed "quantized energy pumping" between $m=2$ different frequencies in $d=0$ spatial dimensions. Let us interpret the topological invariant that was found. In Ref. [59], the limit of very low frequencies was studied, so the adiabatic theorem ensured that the system is always in the ground state of the instantaneous Hamiltonian. The latter varies quasiperiodically, $H(t)=$ $H\left(\boldsymbol{\omega} t+\boldsymbol{\theta}_{0}\right)$ for some continuous function $H(\boldsymbol{\theta})$ defined on the 2-torus $\mathbb{T}^{2}$. Therefore, we can treat the projector onto the ground state of $H(\boldsymbol{\theta})$ as a function of $\boldsymbol{\theta}$, which defines a micromotion that can be classified according to the general framework discussed above. We find that

$$
H^{2}\left(\mathbb{T}^{2}, \mathbb{Z}\right)=\mathcal{H}^{2}(\mathbb{Z} \times \mathbb{Z}, \mathbb{Z})=\mathbb{Z} .
$$

This $\mathbb{Z}$ invariant is the Chern number over the torus $\mathbb{T}^{2}$ of the Berry connection of the ground state. Note that the adiabatic limit considered is very different from the highfrequency one we considered above-in particular, there cannot be a decomposition of the time-evolution operator of the form in Eq. (6) here [41]. However, this difference does not affect the classification. The topological invariants discussed in Ref. [61] can also be understood in a similar fashion.

One might think that the nontrivial invariant of this kind could also be realized in the frame-twisted high-frequency limit through a bootstrap construction as in Sec. V B 1. However, this is not the case because, in the frame-twisted high-frequency limit, the emergent symmetry is always a finite group $\mathcal{G}=\mathbb{Z}^{\times 2} / \mathcal{L}^{\prime}$. One can check that although $H^{2}(\mathbb{Z} \times \mathbb{Z}, \mathbb{Z})=\mathbb{Z}$, the image in this group under the map $\mathcal{H}^{2}(\mathcal{G}, \mathbb{Z}) \rightarrow \mathcal{H}^{2}(\mathbb{Z} \times \mathbb{Z}, \mathbb{Z})$ induced by the projection map $\mathbb{Z} \times \mathbb{Z} \rightarrow \mathcal{G}$ is always trivial for any finite quotient $\mathcal{G}$.

In Ref. [60], "Majorana multiplexing" was introduced, where a system in one spatial dimension that is quasiperiodically driven with $m$ incommensurate frequencies may host $2^{m}$ different kinds of boundary Majorana zero modes that can occur simultaneously, while being protected from coupling to each other. This case is consistent with our general framework since the Majorana modes are distinguished by the charge $( \pm 1)$ they carry under each of the $m$ generators of the $\mathbb{Z}^{\times m}$ time-translation symmetry group. One can readily show that any combination of such boundary Majorana zero modes can be realized in a frametwisted high-frequency limit in a bootstrap construction of the general form described above. In particular, this construction demonstrates that the "Majorana multiplexing" of Ref. [60] can be made stable to interactions, at least up to the parametrically long heating time $t_{*}$, in the presence of strong disorder and driving at high frequencies.

\section{ESTIMATING THE HEATING TIME IN QUASIPERIODICALLY DRIVEN SYSTEMS}

We return to the important question of dynamics and slow heating in quasiperiodically driven systems that underpin the existence of the nonequilibrium phases of matter discussed in the preceding sections. We first give schematic arguments within linear response that can be used to understand the stretched exponential scaling of the heating time $t_{*}$ of Eq. (3), by extending the discussion of Sec. II B. This analysis follows that of Ref. [31]. The scaling of $t_{*}$ that we obtain through these arguments will be borne out in the rigorous bounds on heating of Sec. VII.

\section{A. Linear response arguments}

As discussed in Sec. II B, the heating rate in a quasiperiodically driven system is governed by the competition between the decay of Fourier series coefficients of the driving term $V_{\boldsymbol{n}}$ with $|\boldsymbol{n}|$ and the fact that $|\boldsymbol{\omega} \cdot \boldsymbol{n}| /|\boldsymbol{\omega}|$ could become ever smaller as $|\boldsymbol{n}|$ increases. In order to estimate the heating time, we need to quantify exactly how small $|\boldsymbol{\omega} \cdot \boldsymbol{n}|$ can get as a function of $|\boldsymbol{n}|$. The key mathematical tool is the following Diophantine condition:

$$
\frac{|\boldsymbol{\omega} \cdot \boldsymbol{n}|}{|\boldsymbol{\omega}|} \geq \frac{c}{|\boldsymbol{n}|^{\gamma}},
$$

for all integer vectors $\boldsymbol{n} \neq \mathbf{0}$, where $c$ is a constant depending on the ratios of the frequencies $\omega_{i} / \omega_{j}$ but not on the overall frequency scale $|\omega|$. It can be rigorously shown (see Appendix G) that all choices of frequency vectors, except a set of measure zero, obey such a condition for any $\gamma>m-1$ (with a constant $c$ depending on $\gamma$ ). In particular, Eq. (55) holds (again, for any $\gamma>m-1$, and with $c$ depending on $\gamma$ ) when the ratios $\omega_{i} / \omega_{j}$ are all irrational algebraic numbers - that is, they are each roots of some polynomial equation with integer coefficients, in which case it is known as the subspace theorem $[127,128]$.

In our analysis, we always assume that the frequency vectors $\omega$ obey Eq. (55), which allows us to derive lower bounds on the scaling of the heating time at high frequencies (recall that this high-frequency limit corresponds to taking $\omega:=|\omega| \rightarrow \infty$ while keeping the ratio $\omega_{i} / \omega_{j}$ fixed). 
For the periodically driven case, we can use Eq. (55) with $\gamma=0$.

In linear response, a term $V_{n}$ can drive transitions between energy levels of the average Hamiltonian separated by energy $\Delta E_{\boldsymbol{n}}=\boldsymbol{\omega} \cdot \boldsymbol{n}$. However, such processes require a rearrangement of $\sim \Delta E_{n} / J$ sites, where $J$ is the local strength of the average Hamiltonian; hence, the amplitudes for such processes are suppressed by a factor

$$
e^{-\kappa\left(\Delta E_{n} / J\right)} \leq e^{-\kappa(\omega / J)|\boldsymbol{n}|^{-\gamma}}
$$

for some constant $\kappa>0$, using the Diophantine condition, Eq. (55). Since only $|\boldsymbol{n}| \neq 0$ processes contribute to heating, the smallest value of $|\boldsymbol{n}|$ is 1 , in which case this term evaluates to $e^{-\kappa(\omega / J)}$, an exponentially small factor at high frequencies. For $\gamma=0$ (the periodic case), there is no $\boldsymbol{n}$ dependence on the right-hand side of Eq. (56), and we recover the usual exponential scaling of heating time with frequency. For quasiperiodically driven systems, on the other hand, $\gamma>0$, and then the right-hand side of Eq. (56) goes to 1 at large $|\boldsymbol{n}|$. This result suggests that the heating rate is not suppressed even at high frequencies.

To derive stronger results, the key is to use the smoothness of the driving. If we assume the local strength of $V_{n}$ is bounded by some function $g(|\boldsymbol{n}|)$, then the rate of a heating process governed by a given $V_{n}$ is controlled by both Eq. (56) and $g(|\boldsymbol{n}|)$, which gives a suppression factor

$$
g(|\boldsymbol{n}|)^{2} e^{-\kappa(\omega / J)|\boldsymbol{n}|^{-\gamma}} .
$$

To obtain the full heating rate, we sum over all such processes. Asymptotically, it is governed by the fastest heating rate: For a given form of $g(|\boldsymbol{n}|)$, there will be a value of $|\boldsymbol{n}|$ that maximizes Eq. (57), and the heating time then scales like the inverse of the maximum value of Eq. (57). If $g(|\boldsymbol{n}|)$ decays exponentially in $|\boldsymbol{n}|$, which is the case we consider throughout our paper, then we find $t_{*} \sim \exp \left[\right.$ const $\left.(\omega / J)^{1 /(\gamma+1)}\right]$, which is a scaling similar to the bound in Eq. (3). One sees that unlike the Floquet case, for quasiperiodic driving $(\gamma>0)$, it is crucial to use the smoothness of the driving. Indeed, if $g(|\boldsymbol{n}|)$ does not decay with $|\boldsymbol{n}|$, we see that the heating time does not grow with frequency at all if $\gamma>0$. Treating other forms of drives, where $g(|\boldsymbol{n}|)$ has different asymptotic forms at large $|\boldsymbol{n}|$, is an interesting direction to develop; see Sec. VIII C.

\section{B. Stability to varying the frequencies}

Our results have assumed keeping the ratios $\omega_{i} / \omega_{j}$ constant and only changing the overall frequency scale $|\omega|$ to reach the high-frequency limit. In practice, the physical time evolution of the system should not depend too sensitively on the precise ratios $\omega_{i} / \omega_{j}$ and associated constant $c$. We expect there to be a long timescale before the system can resolve the distinction between a drive with driving frequencies $\boldsymbol{\omega}$ and one with nearby driving frequencies $\boldsymbol{\omega}+\delta \boldsymbol{\omega}$ (for example, a close rational approximation, for which our results technically do not apply).

We can estimate this timescale at the level of the linear response arguments. Consider a vector $\boldsymbol{\omega}$ that satisfies the conditions for our results to hold, Eq. (55), and consider perturbing the frequency $\boldsymbol{\omega} \rightarrow \boldsymbol{\omega}^{\prime}=\boldsymbol{\omega}+\delta \boldsymbol{\omega}$. By rescaling, we can ensure that $\left|\boldsymbol{\omega}^{\prime}\right|=|\boldsymbol{\omega}|$. From the triangle inequality,

$$
\frac{\left|\boldsymbol{\omega}^{\prime} \cdot \boldsymbol{n}\right|}{|\boldsymbol{\omega}|} \geq c|\boldsymbol{n}|^{-\gamma}-\frac{|\delta \boldsymbol{\omega}||\boldsymbol{n}|}{|\boldsymbol{\omega}|} \geq \frac{c}{2}|\boldsymbol{n}|^{-\gamma},
$$

provided that

$$
|\boldsymbol{n}| \leq n_{\max }=\left(\frac{c|\boldsymbol{\omega}|}{2|\delta \boldsymbol{\omega}|}\right)^{1 /(\gamma+1)} .
$$

In other words, the new frequency vector still satisfies the required approximation condition (with a constant $c$ that is independent of $\delta \boldsymbol{\omega}$ ) but only when $|\boldsymbol{n}| \leq n_{\max }$.

In this context, the effect of processes with $|\boldsymbol{n}| \geq n_{\max }$ will never be felt if we truncate the Fourier expansion $V_{n}$ of the driving so that $V_{n}=0$ for $|\boldsymbol{n}|>n_{\max }$. Assuming smooth driving, so that $\left\|V_{\boldsymbol{n}}\right\|$ decays exponentially with $|\boldsymbol{n}|$, then this truncation will not substantially affect the dynamics until a time

$$
t_{\text {perturb }} \sim e^{C n_{\max }}=\exp \left(C\left[\frac{c|\boldsymbol{\omega}|}{2|\delta \boldsymbol{\omega}|}\right]^{1 /(\gamma+1)}\right),
$$

for some constant $C$. After $t_{\text {perturb }}$, the evolution of the system will be governed by a different regime.

Therefore, the worst-case scenario when perturbing the frequency vector is that the heating time $t_{*}$ will grow with frequency until it reaches $t_{\text {perturb }}$ and then stop growing because the system immediately heats up at time $t_{\text {perturb }}$. However, this heating scenario is not inevitable because the dynamics after $t_{\text {perturb }}$ could have a much longer heating time $t_{*}^{\prime}$ that grows with frequency. For example, if $\boldsymbol{\omega}^{\prime}$ is a rationally related frequency vector, then the system is really periodic, and one can invoke Floquet slow heating results.

\section{LONG-LIVED, PREHEATING REGIME IN QUASIPERIODICALLY DRIVEN SYSTEMS: RIGOROUS RESULTS AND SKETCH OF PROOF}

In this section, we formalize the preceding discussions on slow heating and emergent symmetries into a rigorous theorem on preheating in quasiperiodically driven systems. We do this by explicitly constructing the effective Hamiltonian $D$ and providing bounds on its validity. The construction employed also manifestly allows for emergent symmetries to appear in $D$, should the driving Hamiltonian have twisted TTSs as described in Sec. III B. The exact formulation of the proof and heating bounds is somewhat involved, and we leave the technical details to Appendix $\mathrm{H}$. 
Here, we provide an accessible statement of the theorem, as well as an outline of the proof.

\section{A. Conditions and setup}

Recall that we are considering quasiperiodically driven systems defined on lattices in $d$ spatial dimensions with locally bounded Hilbert spaces, i.e., spins or fermions, with a driving frequency vector $\boldsymbol{\omega}$ that is rationally independent, $\boldsymbol{\omega} \cdot \boldsymbol{n} \neq \mathbf{0}$ for any nonzero integer vector $\boldsymbol{n} \in \mathbb{Z}^{m}$. We furthermore assume that the time-quasiperiodic Hamiltonian is quasilocal, with the drive performed at high frequencies, and also smooth in time. What we mean by the high-frequency condition is that each driving frequency $\omega_{i}$ is large compared to the local energy scales $J$ of the Hamiltonian $H(\boldsymbol{\theta})$, and the smoothness of the drive condition is the imposition of the condition that the Fourier modes $H_{\boldsymbol{n}}$ decay exponentially fast with $|\boldsymbol{n}|$, i.e., $\left\|H_{n}\right\|=O\left(e^{-\kappa^{\prime}|\boldsymbol{n}|}\right)$ for some $\kappa^{\prime}>0$. Additionally, let us remind the reader that we assume that the frequency vector $\omega$ obeys the Diophantine condition, Eq. (55), which holds for all choices of rationally independent frequency vectors except for a set of measure zero.

More precisely, our construction and theorem make use of a notion of a local norm $\|O\|_{\kappa}$ parametrized by a constant $\kappa>0$, appropriate for a many-body operator $O(\boldsymbol{\theta})$ parametrized on the torus $\mathbb{T}^{m}$, which acts on an infinite lattice with bounded local Hilbert space dimension. To define this norm, let us first write (nonuniquely) a many-body operator $O(\boldsymbol{\theta})$ in terms of a sum of local "potentials" $O_{Z}(\boldsymbol{\theta})$ on $\mathbb{T}^{m}$, where $Z$ is a finite subset of the lattice and where $O_{Z}(\boldsymbol{\theta})$ only acts nontrivially on sites $x \in Z$ for all $\boldsymbol{\theta} \in \mathbb{T}^{m}$. We can furthermore decompose the local potential into its Fourier modes $O_{Z}(\boldsymbol{\theta})=\sum_{\boldsymbol{n}} O_{Z, \boldsymbol{n} \in \mathbb{Z}^{m}} e^{i \boldsymbol{n} \cdot \boldsymbol{\theta}}$ so that

$$
O(\boldsymbol{\theta})=\sum_{Z} O_{Z}(\boldsymbol{\theta})=\sum_{Z, \boldsymbol{n}} O_{Z, \boldsymbol{n}} e^{i \boldsymbol{n} \cdot \boldsymbol{\theta}} .
$$

We then define $\|O\|_{\kappa}$ to be

$$
\|O\|_{\kappa}=\sup _{x} \sum_{\boldsymbol{n}, Z \ni x} e^{\kappa(|Z|+|\boldsymbol{n}|)}\left\|O_{Z, \boldsymbol{n}}\right\|
$$

for some constant $\kappa>0$, where the supremum is over sites $x$ on the lattice, and the norm appearing in the sum is the standard operator norm.

The norm measures the strength of local terms making up $O(\boldsymbol{\theta})$, specifically taking into account the decay of the strength of interactions in both spatial extent and Fourier space. Terms corresponding to larger spatial support and higher Fourier modes are weighted more, parametrized by $\kappa$, which can be understood as the decay constant. Note that the norm is only really useful if it is finite. Thus, the local terms appearing in the sum in Eq. (61) have to decay at least exponentially fast, both in real and Fourier space. The quasilocal and time-smooth nature of the driving
$H(\boldsymbol{\theta})$ we imposed ensures that there is some $\kappa>0$ for which $\|H\|_{\kappa}<\infty$.

\section{B. Theorem and statement of results: Long-lived, preheating description of dynamics and emergent symmetries}

With this setup, we turn to the object of interest: the unitary time-evolution operator

$$
U(t)=\mathcal{T} \exp \left(-i \int_{0}^{t} H\left(t^{\prime}\right) d t^{\prime}\right)
$$

generated by a quasiperiodically driven Hamiltonian $H(t)=H\left(\boldsymbol{\omega} t+\boldsymbol{\theta}_{0}\right)$ for some $\boldsymbol{\theta}_{0} \in \mathbb{T}^{m}$. We assume there is some decay constant $\kappa_{0}>0$ such that $H(\boldsymbol{\theta})$ has a local norm $J:=\|H\|_{\kappa_{0}}$, which is small enough compared to the driving frequency: $J \leq K \omega$. Here, $K$ is some numerical constant that does not depend on the Hamiltonian, geometry of the lattice, or driving frequencies, which we give explicitly in Appendix $\mathrm{H}$.

We then have the following statements.

(1) Existence of a long-lived preheating dynamical description.-It is possible to find a decomposition of the unitary time-evolution operator as

$U(t)=P(t) \mathcal{T} \exp \left(-i \int_{0}^{t}\left[D+V\left(t^{\prime}\right)\right] d t^{\prime}\right) P^{\dagger}(0)$,

where $P(t)=P\left(\boldsymbol{\omega} t+\boldsymbol{\theta}_{0}\right)$ is a time-quasiperiodic quasilocal unitary, $D$ is a time-independent, quasilocal Hamiltonian, and $V(t)=V\left(\boldsymbol{\omega} t+\boldsymbol{\theta}_{0}\right)$ is a time-quasiperiodic, quasilocal Hamiltonian. The functions $P(\boldsymbol{\theta}), V(\boldsymbol{\theta})$ are smooth on $\mathbb{T}^{m}$.

Define the decay constant $\kappa=\kappa_{0} / 4$. The Hamiltonian $D$ is close to the time-averaged Hamiltonian

$$
\langle H\rangle=\int_{\mathbb{T}^{m}} \frac{d^{m} \boldsymbol{\theta}}{(2 \pi)^{m}} H(\boldsymbol{\theta}),
$$

in the sense $\|D-\langle H\rangle\|_{\kappa} \leq C J(J / \omega)$. The term $V(\boldsymbol{\theta})$ is small, in the sense that

$$
\|V\|_{\kappa} \leq J 2^{-q_{*}}
$$

where

$$
q_{*}=\left\lfloor K^{\prime}\left(\frac{\omega}{J}\right)^{\frac{1}{\gamma+1}}\right\rfloor .
$$

Here, $C, K^{\prime}$ are numerical constants, which we compute in Appendix H; importantly, they do not depend on the Hamiltonian or driving frequencies.

We now spell out the dynamical consequences.

(i) Slow heating.- The time-averaged Hamiltonian $\langle H\rangle$ is an almost conserved energy operator, up to perturbative corrections of order $J / \omega$, captured precisely by 


$$
\frac{1}{\mathrm{Vol}}\left\|U^{\dagger}(t)\langle H\rangle U(t)-\langle H\rangle\right\| \leq J\left(\tilde{K} 2^{-q_{*}} t+C(J / \omega)\right)
$$

for all $t \geq 0$. In the above equation, the standard operator norm is used, and we have divided by the volume "Vol" of the system. In other words, the normalized energy density as measured by $\langle H\rangle / J$ grows very slowly, apart from a small quantity of order $J / \omega$. Here, $\tilde{K}, C$ are finite constants that do not depend on the Hamiltonian, geometry of the lattice, or driving frequencies. An analogous statement holds when replacing $\langle H\rangle \mapsto D$ as they only differ by small terms on the order of $J / \omega$.

From this statement, we can thus derive that the heating time $t_{*}$, defined as the time beyond which the quantity $(1 / \mathrm{Vol})\left\|U^{\dagger}(t)\langle H\rangle U(t)-\langle H\rangle\right\|$ starts growing appreciably, obeys a lower bound

$$
t_{*} \geq \tilde{K}^{-1} 2^{q_{*}} \equiv \frac{C^{\prime}}{J} \exp \left[C\left(\frac{\omega}{J}\right)^{1 /(m+\epsilon)}\right],
$$

for appropriately defined numerical constants $C^{\prime}, C$. Note that this is only a lower bound for $t_{*}$ because Eq. (68) is only an upper bound on how fast the energy density can change.

(ii) Effective description of dynamics. - If we define

$$
\hat{U}(t)=P(t) e^{-i D t} P^{\dagger}(0),
$$

then for any local operator $O$, we have that

$$
\begin{gathered}
\left\|\hat{U}^{\dagger}(t) O \hat{U}(t)-U^{\dagger}(t) O U(t)\right\| \\
\quad \leq K^{\prime \prime}(O) 2^{-q_{*}} J t(1+J t)^{d},
\end{gathered}
$$

where $K^{\prime \prime}(O)$ is a numerical constant not depending on the Hamiltonian or driving frequencies. Thus, the difference, Eq. (71), is very small for times less than the heating time $t_{*} \sim 2^{q_{*}}$.

(2) Existence of emergent symmetries in effective Hamilton D.-If, in addition, the original driving Hamiltonian $H(\boldsymbol{\theta})$ has twisted TTSs generated by $g_{\tilde{\tau}}$ (for some set of $\tilde{\boldsymbol{\tau}}$ s), that is,

$$
H(\boldsymbol{\theta}+\tilde{\boldsymbol{\tau}})=g_{\tilde{\tau}} H(\boldsymbol{\theta}) g_{\tilde{\boldsymbol{\tau}}}^{\dagger},
$$

as described in Sec. III B, then $V(\boldsymbol{\theta}), P(\boldsymbol{\theta})$ in the above decomposition of the unitary also have the same twisted TTSs. The effective Hamiltonian $D$ obeys

$$
\left[D, g_{\tilde{\tau}}\right]=0 .
$$

The above statements form the rigorous basis on which the results of this paper rest. They show that (i) the system does not heat until the long time $t_{*}$, (ii) in the preheating regime, the decomposition Eq. (6) holds up to small corrections that can be ignored until $t_{*}$, in the sense made precise by Eqs. (68) and (71), and (iii) the effective static Hamiltonian $D$ possesses emergent symmetries as a consequence of twisted TTSs of the drive.

\section{Main ideas and sketch of proof}

Here, we provide the ideas underlying our technical procedure that allows us to derive the previous assertions, as well as sketch the proof. To obtain the dynamical statement (1) above, we employ an iterative procedure "renormalizing" the initial driving Hamiltonian $H(\boldsymbol{\theta})$ through a series of small rotations that sequentially reduce the norm of time-dependent pieces. This is possible under conditions of high frequencies where the parameter $J / \omega$, the ratio of the local energy scales to the driving frequency $\omega$, sets a natural small parameter. Upon stopping at some optimal order, we will eventually obtain the effective static Hamiltonian $D$ as well as a remnant, small, time-dependent piece $V(\boldsymbol{\theta})$. We expect that the procedure cannot generically be carried out ad infinitum as this would imply that a driven interacting system has a static local Hamiltonian description, which would go against the unbounded heating we expect to occur at long times.

Such logic is behind the rigorous prethermalization works of Refs. [34,35], and indeed, our manipulations largely closely follow those of Ref. [34] but with a number of technical extensions to handle the quasiperiodically driven scenario. Our main contribution, however, and the biggest departure from the earlier works, is that we employ a renormalization procedure specifically tailored for preserving a twisted TTS at all stages, which then allows us to obtain our statement (2) on emergent symmetries in $D$.

Setting up the iteration.-The renormalization process is nothing but a sequence of well-chosen rotating-frame transformations, effected by the unitaries $P^{(q)}(\boldsymbol{\theta})=P^{(q)}(\boldsymbol{\theta}+\boldsymbol{\tau})$, where $\tau \in \mathcal{L} \in 2 \pi \mathbb{Z}^{m}$ and $q=0,1, \ldots$ up to some cutoff $q_{*}$ to be determined. We start the process by defining the original Hamiltonian as the zeroth-level renormalized Hamiltonian $H^{(0)}(\boldsymbol{\theta}) \equiv H(\boldsymbol{\theta})$. The rotation $P^{(q)}(\boldsymbol{\theta})$ defines the $q$ th level renormalized Hamiltonian at the next level,

$$
H^{(q)}(\boldsymbol{\theta}) \mapsto H^{(q+1)}(\boldsymbol{\theta}):=P^{(q), \dagger}(\boldsymbol{\theta})\left(H^{(q)}(\boldsymbol{\theta})-i \boldsymbol{\omega} \cdot \partial_{\boldsymbol{\theta}}\right) P^{(q)}(\boldsymbol{\theta}) .
$$

Here, $H^{(q)}(\boldsymbol{\theta})$ is the resulting Hamiltonian in the new frame of reference. Let us also define the unitaries

$$
U^{(q)}(t):=\mathcal{T} \exp \left(-\int_{0}^{t} H^{(q)}\left(\boldsymbol{\omega} t+\boldsymbol{\theta}_{0}\right)\right)
$$

in particular, $U^{(0)}(t)=U(t)$, the unitary time-evolution operator of interest. In terms of dynamics, the iterative procedure is 


$$
U^{(q)}(t)=P^{(q)}\left(\boldsymbol{\omega} t+\boldsymbol{\theta}_{0}\right) U^{(q+1)}(t) P^{(q), \dagger}\left(\boldsymbol{\theta}_{0}\right) .
$$

As mentioned, the aim is to reduce the time-dependent terms in $H^{(q)}(\boldsymbol{\theta})$ at each level. To that end, let us define the time-averaging operation

$$
\left\langle H^{(q)}\right\rangle:=\int_{\mathbb{T}^{m}} \frac{d^{m} \boldsymbol{\theta}}{(2 \pi)^{m}} H^{(q)}(\boldsymbol{\theta})=H_{\mathbf{0}}^{(q)},
$$

so that we can write $H^{(q)}(\boldsymbol{\theta})=D^{(q)}+V^{(q)}(\boldsymbol{\theta})$, where

$$
\begin{aligned}
D^{(q)} & =\left\langle H^{(q)}\right\rangle, \\
V^{(q)}(\boldsymbol{\theta}) & =H^{(q)}(\boldsymbol{\theta})-D^{(q)} .
\end{aligned}
$$

We see that we need to eliminate, or at least reduce, the contributions of $V^{(q)}(\boldsymbol{\theta})$ in the next level Hamiltonian $H^{(q+1)}(\boldsymbol{\theta})$.

The high-frequency assumption allows us to choose appropriate rotations $P^{(q)}(\boldsymbol{\theta})$ that are close to the identify ("small"). To gain some intuition, we write $P^{(q)}(\boldsymbol{\theta})=$ $e^{A^{(q)}(\boldsymbol{\theta})}$ for some anti-Hermitian operator $A^{(q)}(\boldsymbol{\theta})$ assumed to be smaller than $V^{(q)}$ by a factor of $1 / \omega$. Then, using the Duhamel formula to expand $H^{(q+1)}(\boldsymbol{\theta})$, we have

$$
\begin{aligned}
H^{(q+1)}(\boldsymbol{\theta})= & D^{(q)}+V^{(q)}(\boldsymbol{\theta})-i \boldsymbol{\omega} \cdot \partial_{\boldsymbol{\theta}} A^{(q)}(\boldsymbol{\theta}) \\
& -\left[A^{(q)}(\boldsymbol{\theta}), H^{(q)}(\boldsymbol{\theta})\right]-\frac{i}{2}\left[A^{(q)}(\boldsymbol{\theta}), \boldsymbol{\omega} \cdot \partial_{\boldsymbol{\theta}} A^{(q)}(\boldsymbol{\theta})\right] \\
& +\cdots,
\end{aligned}
$$

and we see schematically that all terms beyond the first line are smaller than $V^{(q)}$ by a factor of $J / \omega$ or less.

Let us therefore demand that in our iterative procedure, the generators $A^{(q)}(\boldsymbol{\theta})$ are chosen to satisfy

$$
V^{(q)}(\boldsymbol{\theta})-i\left(\boldsymbol{\omega} \cdot \partial_{\boldsymbol{\theta}}\right) A^{(q)}(\boldsymbol{\theta})=0 .
$$

Note that there is a freedom of choice in the solution of this partial differential equation, as the initial condition has not been specified.

So far, the manipulations have been formally identical to that of Ref. [34] upon reduction to the Floquet case. However (and here is the crucial technical difference), we solve the above equation for $A^{(q)}(\boldsymbol{\theta})=\sum_{n} A_{n}^{(q)} e^{i \boldsymbol{n} \cdot \boldsymbol{\theta}}$ in terms of Fourier modes of $V^{(q)}(\boldsymbol{\theta})=\sum_{\boldsymbol{n}} V_{\boldsymbol{n}}^{(q)} e^{i \boldsymbol{n} \cdot \boldsymbol{\theta}}$, with the specific choice

$$
A_{\boldsymbol{n}}^{(q)}= \begin{cases}-\frac{1}{\boldsymbol{\omega} \cdot \boldsymbol{n}} V_{\boldsymbol{n}}^{(q)} & \boldsymbol{n} \neq \mathbf{0} \\ 0 & \boldsymbol{n}=\mathbf{0} .\end{cases}
$$

This solution fixes the initial condition by setting $\left\langle A^{(q)}\right\rangle=0$. In contrast, the immediate generalization of Ref. [34] would be to set $A^{(q)}\left(\boldsymbol{\theta}_{0}\right)=0$. Our different choice of the initial condition is what allows our iterative procedure to preserve twisted TTSs, as we will see later.

Equation (80) highlights the fact that the solution only makes sense if the Fourier modes $V_{\boldsymbol{n}}^{(q)}$ decay fast enough so that $A_{n}^{(q)}$ can be written as a convergent Fourier series and $\|A\|_{\kappa}$ is at least well defined for some values of $\kappa$. This is the technical reason for the imposition of the "smoothness" of drive conditions.

As a consistency check, one can see from Eq. (80) that $A^{(q)}$, loosely speaking, differs from $V^{(q)}$ by a "small" factor of $\sim 1 / \omega$. More precisely, the size of $A^{(q)}$ relative to that of $V^{(q)}$ should be measured by their local norms as given by Eq. (62), with slightly different decay constants $\kappa$ (see Appendix $\mathrm{H}$ for details). Note that the choice Eq. (80) also preserves the quasilocality: If $V^{(q)}(\boldsymbol{\theta})$ is quasilocal, then so is $A^{(q)}(\boldsymbol{\theta})$.

Estimating the optimal orderq ${ }_{*}$ - - Having set up the iteration, let us now present the logic behind bounding how far this iterative procedure can be carried out. While satisfying Eq. (79) makes $H^{(q)}(\boldsymbol{\theta})$ less dependent on $\boldsymbol{\theta}$, there is a price to pay: The extra terms generated at each level in the renormalized Hamiltonians are of a longer range. To account for this result and in order to meaningfully estimate their local strength, we allow for some sequence of strictly decreasing decay constants $\kappa_{0}>\kappa_{1}>\kappa_{2} \cdots>0$, which we have to pick judiciously, and measure the Hamiltonian $H^{(q)}(\boldsymbol{\theta})$ at the $q$ th level through its norm $\left\|H^{(q)}(\boldsymbol{\theta})\right\|_{\kappa_{q}}$. However, at some stage, the smallness of $\kappa_{q}$ will impede our ability to bound the Hamiltonian $H^{(q+1)}(\boldsymbol{\theta})$ at the next level; this stage is when the renormalization procedure stops. The aim is to choose a suitable set of decay constants $\kappa_{q}$, which allows for the inductive process to be carried out to as high an order as possible, rendering the resulting $V^{\left(q_{*}\right)}(\boldsymbol{\theta})$ optimally small and giving the effective Hamiltonian $D:=D^{\left(q_{*}\right)}$ at the stopping order $q_{*}$.

Our proof indeed follows such a procedure. We skip the heavily technical details in setting up various inductive bounds, as well as choosing the appropriate decay constants $\kappa_{q}$, but merely state that we end up with the optimal level of truncation $q_{*}$ as given in Eq. (67). This also yields the claimed bounds on $D, V(\boldsymbol{\theta})$, defined as the optimal $D^{\left(q_{*}\right)}, V^{\left(q_{*}\right)}(\boldsymbol{\theta})$, respectively. The result (64) then follows, with $P(\boldsymbol{\theta}):=\prod_{l=1}^{q_{*}} P^{(l)}(\boldsymbol{\theta})$. We refer the reader to Appendix $\mathrm{H}$ for the full details of our manipulations.

\section{Emergent symmetries in effective Hamiltonian $D$}

Thus far, the above discussion was purely dynamical, as related to statement (1). Now, let us consider statement (2) on the emergent symmetries in $D$.

Our choice of solutions in Eq. (80) explicitly preserves the twisted TTSs of the original driving Hamiltonian $H(\boldsymbol{\theta})$ at every level of the renormalization procedure. This in turn 
allows for them to be manifested as unitary operators that commute with the effective Hamiltonian $D$. To see this, recall that a twisted TTS acts in Fourier space on a Hamiltonian according to $g_{\tilde{\tau}} H_{n} g_{\tilde{\tau}}^{\dagger}=e^{i n \cdot \tilde{\tau}} H_{n}$, so, in particular, $\langle H\rangle=H_{0}=g_{\tilde{\tau}} H_{0} g_{\tilde{\tau}}^{\dagger}$ is symmetric. Now, if the $q$ th level Hamiltonian $H^{(q)}(\boldsymbol{\theta})$ has a twisted TTS, then $V^{(q)}(\boldsymbol{\theta})$ has the same symmetry, while $D^{(q)}=\left\langle H^{(q)}\right\rangle$ commutes with $g_{\tilde{\tau}}$. But from Eq. (80), these properties immediately imply that $A^{(q)}(\boldsymbol{\theta})$ and hence $P^{(q)}(\boldsymbol{\theta})$ will also inherit the twisted TTS of $V^{(q)}(\boldsymbol{\theta})$ and therefore so will $H^{(q+1)}(\boldsymbol{\theta})$. As this is true for every $q$, we end up with the statement that the effective Hamiltonian $D$ obtained at the optimal order $q_{*}$ has emergent symmetries $g_{\tilde{\tau}}$.

\section{EXTENSIONS AND FUTURE DIRECTIONS}

Having demonstrated how one can achieve novel nonequilibrium phases of matter in quasiperiodically driven systems, we now consider extensions and future directions arising from our work. Many of these are directly inspired by the recent development in nonequilibrium Floquet systems.

\section{A. Long-range interactions}

One of the assumptions that allows us to bound heating rates and establish the preheating regime is that the many-body systems we consider have quasilocal interactions - that is, the amplitudes of the interaction terms in the Hamiltonian decay at least exponentially fast with space. The study of prethermalization in Floquet systems suggests that this restriction may be lifted to encompass long-range interactions. References [129,130] have demonstrated slow heating for periodic driving in the presence of two-body power-law interactions, provided that the interactions decay with distance as $\sim 1 / r^{\alpha}$, with $\alpha>d$, where $d$ is the spatial dimension; see also Ref. [131]. The existence of an effective Hamiltonian approximately generating the dynamics in the preheating regime, however, can only be proven for $\alpha>2 d$. We can immediately combine our proof with the approach of Ref. [130] to derive similar results of slow heating and emergent symmetries in quasiperiodically driven systems with power-law interactions. These extensions are particularly valuable for realizing quasiperiodic phases in trapped ion systems, which naturally have long-range interactions.

\section{B. Time-independent systems: Continuous time quasicrystal}

Although we have focused on applications to quasiperiodically driven systems, our theorem also has an intriguing implication for systems with time-independent Hamiltonians. In particular, let $\Gamma_{1}, \ldots, \Gamma_{m}$ be a set of commuting operators, each of which has integer eigenvalues, and let $\boldsymbol{\omega}=\left(\omega_{1}, \ldots, \omega_{m}\right)$ be some rationally independent numbers. Consider the time-independent Hamiltonian

$$
H=H_{0}+V
$$

where

$$
H_{0}=\sum_{i} \omega_{i} \Gamma_{i}
$$

Observe that the time evolution generated by $H_{0}$ is quasiperiodic, $U_{0}(t)=\exp \left(-i H_{0} t\right)=\exp \left(-i t \omega_{i} \Gamma_{i}\right)$, since $U_{0}(t)=U_{0}(\boldsymbol{\omega} t)$, where $U_{0}(\boldsymbol{\theta})=\exp \left(-i \theta_{i} \Gamma_{i}\right)$. If we define the Hamiltonian in the rotating frame generated by $H_{0}$, i.e.,

$$
H_{\text {int }}(t)=U_{0}^{\dagger}(t) V U_{0}(t) .
$$

Then, it also has quasiperiodic time dependence $H_{\text {int }}(t)=$ $H_{\text {int }}(\omega t)$, with

$$
H_{\text {int }}(\boldsymbol{\theta})=U_{0}^{\dagger}(\boldsymbol{\theta}) V U_{0}(\boldsymbol{\theta}) .
$$

We invoke our theorem to construct a rotating-frame transformation $P(\boldsymbol{\theta})$ that generates a time-independent quasilocal effective Hamiltonian $D$, up to corrections that can be ignored until a time $t_{*}$ that scales like a stretched exponential in $|\boldsymbol{\omega}|$. This procedure might not seem very useful since the original Hamiltonian $H$ was already a timeindependent Hamiltonian. However, crucially, $H_{\text {int }}$ has a twisted TTS in the sense discussed in Sec. III B. Indeed, we have that

$H_{\mathrm{int}}(\boldsymbol{\theta}+\boldsymbol{\tau})=g_{\boldsymbol{\tau}} H_{\mathrm{int}}(\boldsymbol{\theta}) g_{\boldsymbol{\tau}}^{\dagger}, g_{\boldsymbol{\tau}}=\exp \left(i \tau_{i} \Gamma_{i}\right)=U_{0}^{\dagger}(\boldsymbol{\tau})$,

but now for any vector $\tau \in \mathbb{R}^{m}$ (thus, we have a continuous twisted TTS rather than a discrete twisted TTS as considered previously). Therefore, our theorem ensures [see Eq. (64)] that the time-evolution operator generated by $H_{\text {int }}(t)$ can be decomposed (ignoring corrections that only become important at times $\left.t \gtrsim t_{*}\right)$ as

$$
U_{\text {int }}(t) \approx P(t) \exp (-i D t) P^{\dagger}(0),
$$

where $D$ has the emergent symmetries $\left[D, g_{\tau}\right]=0$ for all vectors $\tau$ and hence (by taking the limit $\boldsymbol{\tau} \rightarrow 0$ ) $\left[D, \Gamma_{i}\right]=0$ for all $i$. Moreover, $P(t)=P(\boldsymbol{\omega} t)$, where $P(\boldsymbol{\theta})$ obeys the twisted TTS, i.e., $P(\boldsymbol{\theta}+\boldsymbol{\tau})=g_{\boldsymbol{\tau}} P(\boldsymbol{\theta}) g_{\boldsymbol{\tau}}^{\dagger}$. Hence, using the form of $g_{\tau}$, we find that $P(\boldsymbol{\theta})=U_{0}^{\dagger}(\boldsymbol{\theta}) P U_{0}(\boldsymbol{\theta})$, where $P:=P(0)$. Substituting into Eq. (86), we find

$$
\begin{aligned}
U_{\text {int }}(t) & \approx U_{0}^{\dagger}(t) P U_{0}(t) \exp (-i D t) P^{\dagger} \\
& =U_{0}^{\dagger}(t) P \exp \left(-i\left[D+H_{0}\right] t\right) P^{\dagger} .
\end{aligned}
$$


On the other hand, we also know that, by definition, $U_{\text {int }}(t)=U_{0}^{\dagger}(t) e^{-i H t}$. Therefore, we find that

$$
H \approx P\left(D+H_{0}\right) P^{\dagger} .
$$

The right-hand side of Eq. (89) commutes with $P \Gamma_{i} P^{\dagger}$ for $i=1, \ldots, m$. In other words, the Hamiltonian $H$ has an approximate (because it only holds up to time $t_{*}$ ) emergent $\mathrm{U}(1)^{\times m}$ symmetry. The $m=1$ case was already proven in Ref. [12] (though the connection with twisted TTS was not identified).

Let us discuss three applications of this result. First, we can imagine that the emergent $\mathrm{U}(1)^{\times m}$ symmetry is spontaneously broken. In that case, we obtain a continuous time quasicrystal - a time-independent Hamiltonian that spontaneously develops a quasiperiodic response with frequencies $\omega_{1}, \ldots, \omega_{m}$ until the long time $t_{*}$. This result is a generalization of the prethermal continuous time crystal discussed in Ref. [12].

Another application is the topological protection of quantum information. Reference [132] used the $m=1$ version of this result to argue that the decoherence time for a qubit encoded in a Majorana zero mode on the boundary of a one-dimensional topological superconductor could be made exponentially long (in a parameter of the Hamiltonian) at arbitrary energy density, not just in the ground state, even without disorder. Reference [132] also considered a two-dimensional planar code, but they were not able to show a long lifetime in the limit of large system size because the $m=1$ result does not allow for the separate conservation of the number of $e$-type and $m$-type excitations separately. With our new $m>1$ result, we can now ensure that these numbers are separately conserved, which leads to a (stretched) exponentially long lifetime for the encoded qubit, even as the system size goes to infinity.

Finally, let us discuss an application to a lattice model of charged particles in a strong electric field. Consider charged fermions hopping on a $d$-dimensional lattice, and suppose we set $\Gamma_{i}=\sum_{x} x_{i} c_{x}^{\dagger} c_{x}$ for $i=1, \ldots, d$, where the sum is over the positions $x$ of the lattice sites (which we take to be integer vectors), $x_{i}$ is the $i$ th coordinate of $\boldsymbol{x}$, and $c_{x}^{\dagger}$ is the fermion creation operator at position $x$. Then, the Hamiltonian $H_{0}$ of Eq. (82) corresponds to applying an electric field $\boldsymbol{E}=\boldsymbol{\omega}$ to the system. If $|\boldsymbol{E}|$ is much larger than the other local scales in the Hamiltonian, then we can apply our theorem, and we find that there are $i$ emergent symmetries that are conserved up to time $t_{*}$; we can interpret these as dressed versions of the components of the electric dipole moment. The fact that these dipole moments are conserved prevents particles from hopping from one site to another, which we can think of as a manybody, higher-dimensional version of Stark localization [133-135]. The ability to engineer such conserved quantities in a robust manner can lead to a variety of interesting dynamical phenomena, including the recently discussed idea of "Hilbert space shattering" of Refs. [135-138], where the Hilbert space fractures into exponentially many subsectors.

\section{Nonsmooth drives}

In this paper, we have focused on drives that are smooth in time. It would be interesting to further develop our discussions for cases where the Fourier modes of the drive $H_{n}$ decay slower than exponentially with $|\boldsymbol{n}|$. For example, discrete step drives decay as some power law with $|\boldsymbol{n}|$. Within the simple linear response estimates of Sec. VI, it seems natural to expect a heating time $t_{*}$ that scales like a power law with frequency. However, a more careful calculation of the heating processes along the lines of Sec. VII would be valuable.

Reference [64] considered driving a MBL system with a discrete Fibonacci step drive and found two slow heating regimes: one governed by an effective Hamiltonian generated from a high-frequency expansion whose description lasts for power-law times, followed by one where there is a slow logarithmic decay of observables that lasts up to an exponentially long timescale. Some of the phenomenology considered is very different from the one discussed in Sec. II D; establishing the relationship to the work here remains an open question.

On a technical level, we note that extending our results of Sec. VII to the case of power-law decay of Fourier modes is more complex than applying them to the case of powerlaw decay of interactions with distance. Considering our results and those of Ref. [130], we see that the analogs of power-law dependence of Fourier modes are interactions that decay as a power law in the number of sites contained within the support of the interaction. By contrast, Ref. [130] established results only for interactions that decay as a power law in the diameter of the support while still decaying exponentially in the number of sites.

\section{Quasiperiodically driven topological phases beyond eigenstate micromotion}

In Sec. V, we focused on eigenstate micromotions as a diagnostic of nontrivial topological phases in periodically or quasiperiodically driven MBL systems. However, it is known that there are topological Floquet phases that cannot be diagnosed in this way, many of which have particularly fascinating phenomenology. For example, the phases discussed in Refs. [139-142] are characterized by a chiral pumping of quantum information along the onedimensional boundary of a two-dimensional bulk. One expects analogous phenomena in quasiperiodically driven systems, but we leave such developments for future work.

\section{E. Experimental realizations}

Lastly, let us briefly mention the possibility of experimentally realizing the quasiperiodically driven phases of matter we have discussed, in particular, the DTQC phase described in Sec. IV. 
The driving protocol and conditions required, described in Sec. III C, can easily be realized in setups such as in synthetic quantum systems of trapped ions, or in solid-state systems like nitrogen-vacancy (NV) defects in diamond. Both cases give rise to ensembles of coherently interacting effective spin degrees of freedom, which are well isolated from the environment. Appropriate sequences of laser or microwave pulses can be engineered to realize particular drives in these systems. Indeed, these were utilized to realize sequences $[11,13]$ that led to signatures of discrete time crystal phases observed in experiments of a 1D chain of trapped ions [15] and in a dense, disordered, 3D ensemble of NV centers in diamond [16].

Extending these protocols to realize time-quasiperiodic phases is relatively straightforward, although the time dependence of the drives needs to be smooth in time to avoid fast heating. The different platforms offer different comparative advantages. In trapped ions, the ability to tune the range of long-range interactions between spins implies that it should be possible to realize a DTQC in a prethermal regime even in $1 \mathrm{D}$, provided that the power-law exponent satisfies $1<\alpha<2$. On the other hand, one can utilize the fact that NV centers in diamond are grouped by their orientation with respect to the crystallographic axes of diamond. Using interactions within and between multiple groups, one can naturally realize more complicated TQCs, such as the example Hamiltonian in Appendix D 2, giving rise to a $\mathbb{Z}_{2} \times \mathbb{Z}_{2}$ DTQC, which utilizes two collections of spins. Note that in $3 \mathrm{D}$, the dipolar interacting nature of the spins precludes localization, but thermalization is nonetheless slow in heavily disordered samples (precisely, critically slow; see Refs. [14,143,144]). Thus, a long-lived version of a DTQC might still be realizable, protected by critically slow thermalization dynamics.

The dynamical signatures that one would look for would be the appearance of peaks at fractional harmonics of the input drive frequencies in the power spectrum of a local observable. Additionally, these signatures should be robust to small perturbations to the drive protocol, which still preserve its time quasiperiodicity. These signatures would signal the spontaneous breaking of multiple time-translation symmetries of the original drive and is the defining characteristic of the DTQC phase (see Sec. IV). The exact pattern that is manifested in the Fourier harmonics, however, depends on the exact symmetry-breaking pattern, realized using different driving protocols. As there are multiple timetranslation symmetries in quasiperiodic drives, there are a myriad of complex symmetry-breaking patterns, which could be observed even for a given experimental platform.

\section{CONCLUSION}

In this paper, we have shown how interacting quantum many-body systems that are quasiperiodically driven can realize a panoply of long-lived nonequilibrium phases of matter under a general set of driving conditions. These phases are protected by multiple time-translation symmetries, which arise as the driving Hamiltonian derives from a function $H(\boldsymbol{\theta})$ defined on a higher-dimensional torus. They are fundamentally different from phases realizable in static or Floquet systems and add to the richness of the landscape of possible phases of matter realizable in nonequilibrium settings. As an exemplar, we described the phase of matter obtained by the spontaneous breaking of some or all of the multiple timetranslational symmetries - a time quasicrystal. We also gave a classification of the symmetry-protected topological phases of matter achieved in the quasiperiodic setting.

Key to our results was our ability to identify a dynamical regime, a so-called preheating regime, in which the deleterious effects of heating in driven systems was controlled for a parametrically long time, as well as our analysis of how multiple TTSs play out in this regime. We provided a class of general driving conditions that realize prethermal or quasiperiodic many-body localized phases in this regime. We emphasize that their dynamical signatures are universal and robust against small perturbations to the driving protocol, as long as they respect the time quasiperiodicity of the drive: They are thus genuine nonequilibrium phases of matter in interacting many-body quantum systems.

Our results open up exciting new lines of experimental and theoretical research. Indeed, the nonequilibrium phases of matter we have discussed, in particular, time quasicrystals, are immediately directly accessible in the experimental platforms of today. Theoretically, our work establishes new universal structure present in quantum many-body systems in highly out-of-equilibrium settings. What other possible fundamentally nonequilibrium phases of matter remain to be discovered?

\section{ACKNOWLEDGMENTS}

W. W. H. would like to thank S. Choi, D. Sels, and P. J. D. Crowley for helpful discussions. D. V. E. thanks V. Khemani and J. Lin for helpful discussions. We thank M. Serbyn, A. C. Potter, and R. M. Nandkishore for helpful comments on our manuscript. This work was initiated at the Kavli Institute for Theoretical Physics, which is supported by the National Science Foundation under Grant No. NSF PHY-1748958. The Flatiron Institute is a division of the Simons Foundation. W. W. H. and D. V.E. were each supported by the Gordon and Betty Moore Foundation's EPiQS Initiative, Grant No. GBMF4306 (W. W. H.) and GBMF4303 (D. V.E.). W. W. H. acknowledges support from the National University of Singapore (NUS) Development Grant No. AY2019/2020.

\section{APPENDIX A: GENERALIZED FLOQUET- MAGNUS EXPANSION FOR QUASIPERIODICALLY DRIVEN SYSTEMS}

Here, we provide the derivation of a generalized FloquetMagnus expansion for quasiperiodically driven systems driven at high frequencies, introduced and used in the 
main text in Sec. II C for illustrative purposes on how twisted time-translation symmetries enter into the static Hamiltonian $D$ effectively governing the dynamics of the system.

We note two aspects of the following discussion: (1) This expansion is not the naive direct generalization of the Floquet-Magnus expansion to quasiperiodically driven systems, a point we highlight in the derivation. (2) We do not prove any bounds on the validity of the expansion. More precisely, as with the usual Magnus expansion in Floquet systems, we do not expect the series that we write down to be convergent for a many-body system-instead, it is an asymptotic series. For the Floquet case, it is possible to analyze the optimal order to which the series should be truncated [32,33,35], which gives an optimal effective Hamiltonian $D$ and an estimate on the lifetime that the system can be viewed as having dynamics under $D$. However, we do not give such an estimate but merely generate the formal series. That said, the analysis of this series suggests an interesting alternative and complementary derivation of our results, which we reserve for future work.

The logic behind the generalized Floquet-Magnus expansion, or for that matter, the usual Floquet-Magnus expansion in periodically driven systems, simply involves moving into a suitable rotating frame of reference and deriving the rotating-frame Hamiltonian. At high frequencies $\omega=|\omega|$, it is possible to organize this transformation as a perturbative series in $1 / \omega$ and use the freedom endowed by the frame transformation to cancel time-dependent pieces in the rotating-frame Hamiltonian, order by order. This is also the same logic underlying our technical procedure that yields a different effective Hamiltonian $D$; thus, the following expansion should be viewed as a different derivation of an effective description of the system.

To start, we consider a quasiperiodically driven Hamiltonian $H(t)=H\left(\boldsymbol{\omega} t+\boldsymbol{\theta}_{0}\right)$, where $H(\boldsymbol{\theta})$ is a periodic Hamiltonian on the torus. The unitary time-evolution operator obeys the following equation:

$$
i \partial_{t} U(t)=H(t) U(t) .
$$

We move into a new frame of reference effected by the unitary $P(t)=P\left(\boldsymbol{\omega} t+\boldsymbol{\theta}_{0}\right)$, for some periodic $P(\boldsymbol{\theta})$ on the torus. In other words, we decompose the time-evolution operator as $U(t)=P(t) \tilde{U}(t) P(0)^{\dagger}$, where $\tilde{U}(t)$ obeys

$$
\begin{aligned}
i \partial_{t} \tilde{U}(t) & =\left(P^{\dagger}(t) H(t) P(t)-i P^{\dagger}(t) \partial_{t} P(t)\right) \tilde{U}(t) \\
& \equiv D(t) \tilde{U}(t) .
\end{aligned}
$$

We see that time evolution is now generated by the Hamiltonian $D(t)$, which is time quasiperiodic as $D(t)=D\left(\boldsymbol{\omega} t+\boldsymbol{\theta}_{0}\right)$ for a periodic $D(\boldsymbol{\theta})$, which is given explicitly as

$$
D(\boldsymbol{\theta})=P^{\dagger}(\boldsymbol{\theta}) H(\boldsymbol{\theta}) P(\boldsymbol{\theta})-i P^{\dagger}(\boldsymbol{\theta}) \boldsymbol{\omega} \cdot \partial_{\boldsymbol{\theta}} P(\boldsymbol{\theta}) .
$$

We show how $P(\boldsymbol{\theta})$ can be chosen to make $D(t)$ static, i.e., $D(t)=D$.

To that end, we write $P(\boldsymbol{\theta})$ as the exponential of a sum of anti-Hermitian operators,

$$
P(\boldsymbol{\theta})=\exp (\Omega(\boldsymbol{\theta})), \quad \Omega(\boldsymbol{\theta})=\sum_{q=1}^{\infty} \Omega_{q}(\boldsymbol{\theta}),
$$

where we have organized $\Omega$ as a series with terms labeled by $q$. Note that $q$ will track the order of the high-frequency expansion. Now we can write, using Duhamel's formula,

$$
D(\boldsymbol{\theta})=e^{-\operatorname{ad}_{\Omega(\theta)}} H(\boldsymbol{\theta})-i \frac{1-e^{-\operatorname{ad}_{\Omega(\theta)}}}{\operatorname{ad}_{\Omega(\boldsymbol{\theta})}} \boldsymbol{\omega} \cdot \partial_{\boldsymbol{\theta}} \Omega(\boldsymbol{\theta}),
$$

where $\operatorname{ad}_{A} B=[A, B]$. Expanding, we get

$$
\begin{aligned}
& D(\boldsymbol{\theta})=\sum_{q=1}^{\infty} D^{(q)}(\boldsymbol{\theta}), \\
& D^{(q)}(\boldsymbol{\theta})=G^{(q)}(\boldsymbol{\theta})-i \boldsymbol{\omega} \cdot \partial_{\boldsymbol{\theta}} \boldsymbol{\Omega}_{q+1}(\boldsymbol{\theta}), \\
& G^{(q)}(\boldsymbol{\theta})=\sum_{k=1}^{q} \frac{(-1)^{k}}{k !} \sum_{\substack{1 \leq i_{1}, \ldots, i_{i} \leq q \\
i_{1}+\cdots+i_{k}=q}} \operatorname{ad}_{\Omega_{i_{1}}} \cdots \operatorname{ad}_{\Omega_{i_{k}}} H(\boldsymbol{\theta}) \\
& +i \sum_{m=1}^{q} \sum_{k=1}^{q+1-m} \frac{(-1)^{k+1}}{(k+1) !} \\
& \times \sum_{\substack{1 \leq i_{1}, \ldots, i_{k} \leq q+1-m \\
i_{1}+\cdots+i_{k}=q+1-m}} \operatorname{ad}_{\Omega_{i_{1}}} \cdots \operatorname{ad}_{\Omega_{i_{k}}} \boldsymbol{\omega} \cdot \partial_{\boldsymbol{\theta}} \Omega_{m}(\boldsymbol{\theta}) .
\end{aligned}
$$

The question now is how best to reduce the $\theta$-dependent pieces of $D(\boldsymbol{\theta})$. One natural way is to assume that $\Omega_{q}(\boldsymbol{\theta})$ has norm of $\sim 1 / \omega^{q}$, i.e., treat it as a high-frequency expansion. Therefore, we need to pick the generators $\Omega_{q+1}(\boldsymbol{\theta})$ in such a way that this assumption holds at every step. Indeed, if we write $G^{(q)}(\boldsymbol{\theta})$ in terms of a Fourier series

$$
G^{(q)}(\boldsymbol{\theta})=G_{\mathbf{0}}^{(q)}+\sum_{\boldsymbol{n} \neq \mathbf{0}} G_{\boldsymbol{n}}^{(q)} e^{i \boldsymbol{n} \cdot \boldsymbol{\theta}}
$$

then we see that by imposing the condition

$$
\sum_{n \neq \boldsymbol{0}} G_{n}^{(q)} e^{i n \cdot \boldsymbol{\theta}}-i \boldsymbol{\omega} \cdot \partial_{\boldsymbol{\theta}} \Omega_{q+1}(\boldsymbol{\theta})=0,
$$

the norm of $\Omega_{q+1}$ is smaller than $\Omega_{q}$ by $1 / \omega$ since $\left\|\Omega_{q+1}\right\| \sim\left\|G^{(q)}\right\| /|\boldsymbol{\omega}|$ and $\left\|G^{(q)}\right\| \sim 1 / \omega^{q}$, schematically.

The imposition of Eq. (A8) thus defines a family of expansions, differing by a choice of initial conditions. Indeed, the immediate, direct generalization of the FloquetMagnus expansion to quasiperiodically driven systems 
corresponds to one particular choice: One solves Eq. (A8) with the condition that $\left.\Omega_{q}\left(\boldsymbol{\omega} t+\boldsymbol{\theta}_{0}\right)\right|_{t=0}=0$, so $\left.P(t)\right|_{t=0}=$ $\left.P\left(\boldsymbol{\omega} t+\boldsymbol{\theta}_{0}\right)\right|_{t=0}=\mathbb{I}$. This choice gives the solution

$$
\Omega_{q+1}(\boldsymbol{\theta})=-\sum_{\boldsymbol{n} \neq \mathbf{0}} \frac{G_{\boldsymbol{n}}^{(q)}}{\boldsymbol{n} \cdot \boldsymbol{\omega}}\left(e^{i \boldsymbol{n} \cdot \boldsymbol{\theta}}-e^{i \boldsymbol{n} \cdot \boldsymbol{\theta}_{0}}\right) .
$$

To see why this solution yields the "standard" expansion, for a Floquet system we get, from the above, $P(0)=P(T)=\mathbb{I}$, so at stroboscopic times $t=N T$ for $N \in \mathbb{Z}$, we have the familiar relation $U(N T)=e^{-i D N T}$, ignoring questions of convergence.

Instead, we choose to solve Eq. (A8) as

$$
\Omega_{q+1}(\boldsymbol{\theta})=-\sum_{\boldsymbol{n} \neq \mathbf{0}} \frac{G_{\boldsymbol{n}}^{(q)}}{\boldsymbol{n} \cdot \boldsymbol{\omega}} e^{i \boldsymbol{n} \cdot \boldsymbol{\theta}} .
$$

This solution uniquely defines our "generalized FloquetMagnus" expansion.

To see what terms emerge in our expansion, consider $q=0$. We get, from Eqs. (A6) and (A8),

$$
D^{(0)}(\boldsymbol{\theta})=H_{\mathbf{0}}+\sum_{\boldsymbol{n} \neq \mathbf{0}} H_{\boldsymbol{n}} e^{i \boldsymbol{n} \cdot \boldsymbol{\theta}}-i \boldsymbol{\omega} \cdot \partial_{\boldsymbol{\theta}} \Omega_{1}(\boldsymbol{\theta})=H_{\mathbf{0}}
$$

since $\Omega_{1}(\boldsymbol{\theta})$ is chosen to eliminate $\theta$-dependent pieces, given explicitly by $\Omega_{1}(\boldsymbol{\theta})=-\sum_{\boldsymbol{n} \neq \boldsymbol{0}}\left(H_{\boldsymbol{n}}^{(q)} / \boldsymbol{n} \cdot \boldsymbol{\omega}\right) e^{i \boldsymbol{n} \cdot \boldsymbol{\theta}}$. For $q=1$, we get

$$
\begin{aligned}
D^{(1)}(\boldsymbol{\theta}) & =-\operatorname{ad}_{\Omega_{1}} H(\boldsymbol{\theta})+\frac{i}{2} \operatorname{ad}_{\Omega_{1}} \boldsymbol{\omega} \cdot \partial_{\boldsymbol{\theta}} \Omega_{1}(\boldsymbol{\theta})-i \boldsymbol{\omega} \cdot \partial_{\boldsymbol{\theta}} \Omega_{2}(\boldsymbol{\theta}) \\
& =\frac{1}{2} \sum_{\boldsymbol{n}} \frac{\left[H_{n}, H_{-n}\right]}{\boldsymbol{n} \cdot \boldsymbol{\omega}}
\end{aligned}
$$

since $\Omega_{2}(\boldsymbol{\theta})$ is similarly chosen to eliminate the $\theta$ dependent pieces. In a similar fashion, $D^{(q)}(\boldsymbol{\theta})$ can be derived for any $q$.

Lastly, let us explain how our choice of solution, Eq. (A10), giving our generalized Floquet-Magnus expansion, manifestly allows for twisted time-translation symmetries to be preserved: If the original driving Hamiltonian $H(\boldsymbol{\theta})$ has a twisted time-translational symmetry, that is, if there exists a unitary operator $g$ and integer $N$ satisfying $g^{N}=\mathbb{I}$, as well as some vector $\tilde{\boldsymbol{\tau}}$ on the torus such that $H(\boldsymbol{\theta}+\tilde{\boldsymbol{\tau}})=g H(\boldsymbol{\theta}) g^{\dagger}$, then $D(\boldsymbol{\theta})$ also possesses the same twisted time-translation symmetry. To see this, recall that, on the Fourier modes, a twisted TTS acts as $g H_{n} g^{\dagger}=e^{i n \cdot \tilde{\tau}} H_{n}$. Now, if we assume $G^{(q)}(\boldsymbol{\theta})$ has a twisted TTS for some $q$, then our choice of $\Omega_{q+1}(\boldsymbol{\theta})$, Eq. (A10), will also inherit the same twisted TTS [one just sees that the Fourier modes of $\Omega_{q+1}(\boldsymbol{\theta})$ obey the required transformation]. Consequently, $G^{(q+1)}(\boldsymbol{\theta})$, given by sums of conjugations of $H(\boldsymbol{\theta})$ with various $\Omega_{k}(\boldsymbol{\theta})$ with $k<q$, etc., [see Eq. (A6)], will also have the same twisted TTS. Note that the standard Floquet-Magnus expansion corresponding to a choice, Eq. (A9), does not have this property.

\section{APPENDIX B: PERIODICITY OF $U_{0}(\theta)$ AND RELATED DETAILS}

Here, we prove the claim we made in Sec. III C; namely, if we write $H_{0}(t)=H_{0}\left(\boldsymbol{\theta}_{0}+\boldsymbol{\omega} t\right), H_{0}(\boldsymbol{\theta})=f_{i}(\boldsymbol{\theta}) \Gamma_{i}$, where $\bar{f}_{i}=Q_{i j} \omega_{j}$, then it follows that the time-evolution operator $U_{0}(t)$ generated by $H_{0}(t)$ can be written in the form $U_{0}(t)=U_{0}\left(\boldsymbol{\theta}_{0}+\boldsymbol{\omega} t\right)$, where

$$
U_{0}(\boldsymbol{\theta})=\exp \left(-i h_{i}(\boldsymbol{\theta}) \Gamma_{i}\right)
$$

for some functions $h_{i}(\boldsymbol{\theta})$ to be determined, which will satisfy certain symmetry properties. First, we observe that this amounts to the statement that

$$
U_{0}^{\dagger}(\boldsymbol{\theta})\left(\boldsymbol{\omega} \cdot \partial_{\boldsymbol{\theta}}\right) U_{0}(\boldsymbol{\theta})=-i H_{0}(\boldsymbol{\theta}),
$$

which in turn is equivalent to

$$
\left(\boldsymbol{\omega} \cdot \partial_{\boldsymbol{\theta}}\right) h_{i}(\boldsymbol{\theta})=f_{i}(\boldsymbol{\theta}) .
$$

If we define $\eta_{i}(\boldsymbol{\theta})=f_{i}-\bar{f}_{i}$ and $\Delta_{i}(\boldsymbol{\theta})=h_{i}(\boldsymbol{\theta})-Q_{i j} \theta_{j}$, then Eq. (B3) reduces to

$$
\left(\boldsymbol{\omega} \cdot \partial_{\boldsymbol{\theta}}\right) \Delta_{i}(\boldsymbol{\theta})=\eta_{i}(\boldsymbol{\theta}) .
$$

The solution to this equation can be obtained in terms of the Fourier transform; if we write $\eta_{i}(\boldsymbol{\theta})=\sum_{\boldsymbol{n} \neq 0} e^{i \boldsymbol{n} \cdot \boldsymbol{\theta}} \eta_{i}(\boldsymbol{n})$ and similarly for $\Delta_{i}$, then we can set $\Delta_{i}(\boldsymbol{n})=(\text { in } \cdot \boldsymbol{\omega})^{-1} \eta_{i}(\boldsymbol{n})$ for $\boldsymbol{n} \neq 0$. In conclusion, we have found that

$$
h_{i}(\boldsymbol{\theta})=Q_{i j} \theta_{j}+\Delta_{i}(\boldsymbol{\theta}),
$$

where $\Delta_{i}(\boldsymbol{\theta})$ satisfies $\Delta_{i}(\boldsymbol{\theta}+\boldsymbol{\tau})=\Delta_{i}(\boldsymbol{\theta})$ for all $\boldsymbol{\tau} \in \mathcal{L}$. These functions $h_{i}(\boldsymbol{\theta})$ indeed satisfy the symmetry property claimed in the main text.

\section{APPENDIX C: PROPERTIES OF RATIONAL MATRICES}

Let $Q$ be some $r \times m$ matrix with rational entries. Let $\mathcal{L}$ be the lattice comprising all vectors of the form $2 \pi n$ for some integer vector $\boldsymbol{n}$. We are interested in determining the sublattice $\mathcal{L}^{\prime} \leq \mathcal{L}$ comprising those vectors $\boldsymbol{\tau}^{\prime} \in \mathcal{L}$ such that $e^{i Q_{i j} \tau_{j}^{\prime}}=1$.

To solve this problem, we invoke the well-known fact that any integer matrix $Z$ has a Smith normal form decomposition $Z=V D W$, where $V$ and $W$ are unimodular integer matrices (that is, they are invertible integer matrices whose inverses are also integer matrices) and $D$ is a diagonal integer matrix. Since any rational matrix can be converted to an integer matrix simply by multiplying by 
some integer, we conclude that there is also a Smith normal form for rational matrices: $Q=V D W$, where $V$ and $W$ are still unimodular integer matrices, and $D$ is a diagonal rational matrix.

The Smith decomposition allows us to reduce the problem to the case where $Q$ is diagonal. Suppose that $Q=D=\operatorname{diag}\left(p_{1} / q_{1}, \ldots, p_{\min (r, m)} / q_{\min (r, m)}\right)$, where each $p_{i}, q_{i}$ is a coprime integer (with $q_{i}$ positive and $p_{i}$ nonnegative). We can choose to set $q_{i}=1$ if $p_{i}=0$, and we also define $q_{i}=1$ for $r<i \leq m$. Then, we see that $\mathcal{L}^{\prime}$ comprises an integer linear combination of the $m$ vectors spanned by $\boldsymbol{\tau}^{(i)}=q_{i} \boldsymbol{e}_{i}$ (no summation), for $\left(\boldsymbol{e}^{(i)}\right)_{j}=\delta_{i j}$. More generally, if $Q=V D W$, then $\mathcal{L}^{\prime}$ comprises integer linear combinations of the vectors $W^{-1} \tau^{(i)}$. We also find that $\mathcal{L} / \mathcal{L}^{\prime} \cong \mathbb{Z}_{q_{1}} \times \cdots \times \mathbb{Z}_{q_{m}}$.

Note that a particularly simple case is where $r=m$ and $Q=Z^{-1}$ for some (not necessarily unimodular) integer matrix $Z$. In that case, one finds that all the $p_{i}$ 's are 1 , the $q_{i}$ 's are the diagonal entries of the Smith decomposition of $Z$, and $\mathcal{L}^{\prime}$ simply comprises integer linear combinations of the columns of $Z$.

\section{APPENDIX D: MORE EXAMPLES OF DTQC}

Here, we provide the quasiperiodically driven Hamiltonians realizing the $\mathbb{Z}_{2} \times \mathbb{Z}_{2}$ and $\mathbb{Z}_{3} \times \mathbb{Z}_{2}$ DTQCs shown in Figs. 5 and 6 of Sec. IV.

\section{1. $\mathbb{Z}_{2} \times \mathbb{Z}_{2}$ DTQC}

The model under consideration is a direct extension of the one considered in Sec. IV B, but now it involves two groups of spins. Concretely, suppose we have a system comprised of two subsystems $A, B$. Each subsystem consists of spin- $1 / 2$ degrees of freedom placed along either a $1 \mathrm{D}$ chain or a $2 \mathrm{D}$ square lattice, and we assume that the two subsystems $A, B$ are stacked on top of each other (so that site $i$ of subsystem $A$ is directly above site $i$ of subsystem $B$ ).

Consider the following $m=2$ quasiperiodic Hamiltonian

$$
H(t)=H_{0}(\boldsymbol{\omega} t)+V
$$

where now

$$
V=\sum_{\substack{i<j \\ \alpha=A, B}} J_{i j} \sigma_{i, \alpha}^{z} \sigma_{j, \alpha}^{z}+\sum_{i \leq j} J_{i j}^{\prime} \sigma_{i, A}^{z} \sigma_{j, B}^{z}+\sum_{i, \alpha=A, B} h \sigma_{i, \alpha}^{z} .
$$

Similarly to the Hamiltonian in Eq. (30), Eq. (D2) describes pairwise Ising interactions of spins between and within subsystems, and a longitudinal field in the $z$ direction with strength $h \ll \omega_{i} / 2$. The interactions have strengths $J_{i j}, J_{i j}^{\prime}$, and we consider the $2 d$ case where $J_{i j}=-J \delta_{\langle i, j\rangle}$, where $\langle i, j\rangle$ represent nearest-neighbor pairs of sites on one subsystem, and $J_{i j}^{\prime}=-J \delta_{i, j}$. We take $0<J<\omega_{i} / 2$.
The driving Hamiltonian is taken to be

$$
H_{0}(\boldsymbol{\theta})=\sum_{i, \alpha=A, B} \frac{1}{2}\left(\sigma_{i, \alpha}^{x}+1\right) f_{\alpha}(\boldsymbol{\theta}),
$$

and we choose

$$
\begin{aligned}
& f_{A}(\boldsymbol{\theta})=\pi \omega_{1} \Delta_{N}\left(\theta_{1}\right), \\
& f_{B}(\boldsymbol{\theta})=\pi \omega_{2} \Delta_{N}\left(\theta_{2}\right),
\end{aligned}
$$

where $\Delta_{N}$ is the approximation to the Dirac delta comb introduced in Eq. (35). These functions satisfy $\bar{f}_{i}=Q_{i j} \omega_{j}$, with $Q=\frac{1}{2}\left(\begin{array}{ll}1 & 0 \\ 0 & 1\end{array}\right)$. One can understand this driving protocol as two Floquet drives, performed at frequencies $\omega_{1}, \omega_{2}$, acting on different halves of the system. Indeed, if the two subsystems are disjoint, this protocol would simply result in two independent prethermal discrete time crystals. In the present case, the system has interactions between the different subsystems-nevertheless, we now show that a stable DTQC phase will be realized.

One can easily work out that the interaction Hamiltonian $H_{\text {int }}(\boldsymbol{\theta})$ in the frame-twisted high-frequency limit has periodicity on the lattice $\mathcal{L}^{\prime}$ generated by the translation vectors $\tau_{1}=2 \pi(2,0)$ and $\tau_{2}=2 \pi(0,2)$. In other words, $\mathcal{L}^{\prime}=2 \mathcal{L}=4 \pi \mathbb{Z}^{m}$. Thus, the group of symmetries of the effective Hamiltonian $D$ is $\mathcal{G}=\mathbb{Z}_{2} \times \mathbb{Z}_{2}$. Explicitly, the unitary symmetries realized are $g_{\tilde{\tau}}=\prod_{i} \sigma_{i, A}^{x}$ for $\tilde{\boldsymbol{\tau}}=2 \pi(1,0)$, and $g_{\tilde{\boldsymbol{\tau}}}=\prod_{i} \sigma_{i, B}^{x}$ for $\tilde{\boldsymbol{\tau}}=2 \pi(0,1)$.

Repeating the same analysis as in Sec. IV B, the leadingorder effective Hamiltonian is

$$
D^{(0)}=\sum_{\substack{i<j \\ \alpha=A, B}} J_{i j}\left(a_{\alpha}(N) \sigma_{i, \alpha}^{z} \sigma_{j, \alpha}^{z}+b_{\alpha}(N) \sigma_{i, \alpha}^{y} \sigma_{j, \alpha}^{y}\right),
$$

where $a_{\alpha}(N), b_{\alpha}(N)$ are numerical factors that result from the averaging of the interaction Hamiltonian over the larger unit cell. For $N=20, a_{\alpha}(20)=0.928$ and $b_{\alpha}(20)=0.072$ for $\alpha=A, B$. We observe the following salient features: First, $D^{(0)}$ is manifestly $\mathbb{Z}_{2} \times \mathbb{Z}_{2}$ symmetric. In particular, the $\sigma_{i, A}^{z} \sigma_{i, B}^{z}$ interactions between the two subsystems, which are odd under both $\mathbb{Z}_{2}$ groups, have been eliminated. In fact, any interactions that remain between the subsystems, even at higher orders, must be $\mathbb{Z}_{2} \times \mathbb{Z}_{2}$ symmetric. Second, the Ising interactions in the $z$ direction dominate, in both subsystems.

Thus, an initial state that has sufficiently low energy density with respect to $D^{(0)}$ will equilibrate to a thermal state $\rho_{\beta}$, which spontaneously breaks both $\mathbb{Z}_{2} \times \mathbb{Z}_{2}$ Ising symmetries. Then, $\rho(\boldsymbol{\theta})$ will have periodicity on the lattice $\mathcal{L}_{\mathrm{SSB}}=\mathcal{L}^{\prime}$, whose reciprocal lattice $\mathcal{L}_{\mathrm{SSB}}^{*}$ is generated by the vectors $\boldsymbol{\alpha}_{1}=(1 / 2,0)$ and $\boldsymbol{\alpha}_{2}=(0,1 / 2)$. The power spectrum of the expectation value in time of local operators measured in this state will then exhibit peaks at frequencies 


$$
\Omega=\frac{1}{2} \boldsymbol{n} \cdot \boldsymbol{\omega}, \quad \boldsymbol{n} \in \mathbb{Z}^{2},
$$

which is the manifestation of the spontaneous breaking of the TTSs of the driving Hamiltonian.

In the high-frequency limit, we can treat $\mathcal{V}(t)=\mathbb{I}$ and derive analytic expressions for the measurement of an observable in time $s(\boldsymbol{\theta})=\operatorname{Tr}[\hat{s} \rho(\boldsymbol{\theta})]$. For example, for the observable $\hat{s}=(1 / \mathrm{Vol}) \sum_{i} \sigma_{i, A}^{z}+(1 / \mathrm{Vol}) \sum_{i} \sigma_{i, B}^{z}$, we get

$$
\begin{aligned}
s(\boldsymbol{\theta}) \approx & \frac{1}{\operatorname{Vol}} \sum_{i} \operatorname{Tr}\left[\sigma_{i, A}^{z} \rho_{\beta}\right] \cos \left[\pi \Theta_{N}\left(\theta_{1}\right)\right] \\
& +\frac{1}{\operatorname{Vol}} \sum_{i} \operatorname{Tr}\left[\sigma_{i, A}^{y} \rho_{\beta}\right] \sin \left[\pi \Theta_{N}\left(\theta_{1}\right)\right] \\
& +\frac{1}{\operatorname{Vol}} \sum_{i} \operatorname{Tr}\left[\sigma_{i, B}^{z} \rho_{\beta}\right] \cos \left[\pi \Theta_{N}\left(\theta_{2}\right)\right] \\
& +\frac{1}{\operatorname{Vol}} \sum_{i} \operatorname{Tr}\left[\sigma_{i, B}^{y} \rho_{\beta}\right] \sin \left[\pi \Theta_{N}\left(\theta_{2}\right)\right],
\end{aligned}
$$

where the function $\Theta_{N}(\theta)$ is given by Eq. (41). We plot this observable in Fig. 5 using $\operatorname{Tr}\left[\sigma_{i, \alpha}^{z} \rho_{\beta}\right]=0.9$ and $\operatorname{Tr}\left[\sigma_{i, \alpha}^{y} \rho_{\beta}\right]=0$.

\section{2. $\mathbb{Z}_{3} \times \mathbb{Z}_{2}$ DTQC}

Next, we present the Hamiltonian for the $\mathbb{Z}_{3} \times \mathbb{Z}_{2}$ DTQC. Consider, like in the previous example, a system comprised of two subsystems $A, B$ of square lattices in $d=2$, stacked so that sites lie on top of each other. Assume now, however, that the local degrees of freedom of subsystem $A$ (which reside on the sites) are comprised of three levels (i.e., a spin-1) and those of subsystem $B$ are comprised of two levels (i.e., a spin-1/2). Take the $m=2$ quasiperiodic Hamiltonian to be

$$
H=H_{0}(\omega t)+V,
$$

with

$$
\begin{aligned}
V= & -\sum_{\langle i j\rangle} J^{A}\left(\mu_{i}^{\dagger} \mu_{j}+\text { H.c. }\right)-\sum_{\langle i j\rangle} J^{B} \sigma_{i}^{z} \sigma_{j}^{z} \\
& +\sum_{i} J^{A B}\left(\mu_{i} \sigma_{i}^{z}+\text { H.c. }\right)+\sum_{i} h \sigma_{i}^{z}+h^{\prime}\left(\mu_{i}+\mu_{i}^{\dagger}\right),
\end{aligned}
$$

where the operators $\mu, \eta$ that act locally on $A$ are given explicitly by

$$
\mu=\left(\begin{array}{ccc}
1 & 0 & 0 \\
0 & e^{i 2 \pi / 3} & 0 \\
0 & 0 & e^{i 4 \pi / 3}
\end{array}\right), \quad \eta=\left(\begin{array}{lll}
0 & 1 & 0 \\
0 & 0 & 1 \\
1 & 0 & 0
\end{array}\right) .
$$

These operators are the so-called "clock operators" that enter into quantum clock models: $\eta$ increments the state of a "clock" (the levels in which the matrix $\mu$ is diagonal) one step in a cyclic fashion. Thus, $V$ describes ferromagnetic Ising-like interactions between degrees of freedom of $A$, and also ferromagnetic Ising interactions between degrees of freedom of $B$. We take the driving Hamiltonian to be

$H_{0}=\sum_{i} \frac{1}{3}\left(\left[1-e^{i \frac{2 \pi}{3}}\right] \eta+\right.$ H.c. $) f_{A}(\boldsymbol{\theta})+\sum_{i} \frac{1}{2}\left(\sigma_{i}^{x}+1\right) f_{B}(\boldsymbol{\theta})$,

with

$$
\begin{aligned}
& f_{A}(\boldsymbol{\theta})=\frac{2 \pi \omega_{1}}{3} \Delta_{N}\left(\theta_{1}\right), \\
& f_{B}(\boldsymbol{\theta})=\pi \omega_{1} \Delta_{N}\left(\theta_{1}\right)+\pi \omega_{2} \Delta_{N}\left(\theta_{2}\right),
\end{aligned}
$$

which satisfy $\bar{f}_{i}=Q_{i j} \omega_{j}$, with $Q=\left(\begin{array}{cc}(1 / 3) & 0 \\ (1 / 2) & (1 / 2),\end{array}\right)$. We assume the driving frequencies are sufficiently large compared to all local couplings.

We now take the frame-twisted high-frequency limit. The interaction Hamiltonian $H_{\text {int }}(\boldsymbol{\theta})$ has periodicity on the lattice $\mathcal{L}^{\prime}$ generated by the translation vectors $\tau_{1}=$ $2 \pi(3,1)$ and $\tau_{2}=2 \pi(0,2)$. Therefore, the symmetries of the effective Hamiltonian $D$ belong to the group $\mathcal{G}=\mathbb{Z}_{6} \cong \mathbb{Z}_{3} \times \mathbb{Z}_{2}$. Viewing $\mathcal{G}$ as $\mathbb{Z}_{3} \times \mathbb{Z}_{2}$, it can be checked that the group is explicitly generated by the unitary symmetries $g_{\tilde{\boldsymbol{\tau}}}=\prod_{i} \eta_{i}$ for $\tilde{\boldsymbol{\tau}}=2 \pi(1,1)$ satisfying $\left(\prod_{i} \eta_{i}\right)^{3}=\mathbb{I}$ and $g_{\tilde{\boldsymbol{\tau}}}=\prod_{i} \sigma_{i}^{x}$ for $\tilde{\boldsymbol{\tau}}=2 \pi(0,1)$ satisfying $\left(\prod_{i} \sigma_{i}^{x}\right)^{2}=\mathbb{I}$.

Repeating the same analysis as before (with $N=20$ ), the leading-order effective Hamiltonian $D^{(0)}$ can be computed as

$$
\begin{aligned}
D^{(0)}= & -\sum_{\langle i j\rangle} 0.9280 J^{A}\left(\mu_{i}^{\dagger} \mu_{j}+\text { H.c. }\right) \\
& -\sum_{\langle i j\rangle} J^{B}\left(0.866 \sigma_{i}^{z} \sigma_{j}^{z}+0.134 \sigma_{i}^{y} \sigma_{j}^{y}\right)+\delta,
\end{aligned}
$$

where $\delta$ is a small term (compared to the dominant interactions above), given explicitly by

$$
\begin{aligned}
\delta= & J^{A}\left[-0.018\left(\mu_{i}\left(\mu_{j}^{\dagger} \eta_{j}\right)+\left(\mu_{i}^{\dagger} \eta_{i}\right) \mu_{j}+\text { H.c. }\right)\right. \\
& +0.036\left(\left(\mu_{i} \eta_{i}\right)\left(\eta_{j}^{\dagger} \mu_{j}^{\dagger}\right)+\left(\eta_{i}^{\dagger} \mu_{i}\right)\left(\mu_{j}^{\dagger} \eta_{j}\right)+\text { H.c. }\right) \\
& -(0.018-0.03 i)\left(\left(\mu_{i} \eta_{i}\right)\left(\mu_{j}^{\dagger} \eta_{j}\right)+\left(\mu_{i}^{\dagger} \eta_{i}\right)\left(\mu_{j} \eta_{j}\right)\right)+\text { H.c. } \\
& \left.+(0.009-0.016 i)\left(\mu_{i}^{\dagger}\left(\mu_{j} \eta_{j}\right)+\left(\mu_{i} \eta_{i}\right) \mu_{j}^{\dagger}\right)+\text { H.c. }\right] .
\end{aligned}
$$

It can be explicitly checked that $D^{(0)}$ is $\mathbb{Z}_{3} \times \mathbb{Z}_{2}$ symmetric and that the dominant terms are the interactions $\mu_{i}^{\dagger} \mu_{j}$ on subsystem $A$ and Ising terms $\sigma_{i}^{z} \sigma_{j}^{z}$ on subsystem $B$. Therefore, we expect that the steady state $\rho_{\beta}$ of this system, in the preheating regime, should exhibit spontaneous 
breaking of both $\mathbb{Z}_{3} \times \mathbb{Z}_{2}$ symmetries. Then, $\rho(\boldsymbol{\theta})$ has periodicity on the lattice $\mathcal{L}_{\mathrm{SSB}}=\mathcal{L}^{\prime}$, whose reciprocal lattice $\mathcal{L}_{\mathrm{SSB}}^{*}$ is generated by the vectors $\boldsymbol{\alpha}_{1}=(1 / 3,0)$ and $\boldsymbol{\alpha}_{2}=$ $(-1 / 6,1 / 2)$. The power spectrum of the measurement in time of local operators at long and late times will then exhibit peaks at frequencies

$$
\Omega=n_{1} \frac{\omega_{1}}{3}+n_{2}\left(-\frac{\omega_{1}}{6}+\frac{\omega_{2}}{2}\right),
$$

with $n_{1}, n_{2} \in \mathbb{Z}$.

In the high-frequency limit, we can treat $\mathcal{V}(t)=\mathbb{I}$ and derive analytic expressions for the measurement of an observable in time $s(\boldsymbol{\theta})=\operatorname{Tr}[\hat{s} \rho(\boldsymbol{\theta})]$. For example, for the local observable $\hat{s}=\mu_{i}+\mu_{i}^{\dagger}+\sigma_{i}^{z}$,

$$
\begin{aligned}
s(\boldsymbol{\theta}) \approx & \operatorname{Tr}\left[\sigma_{i}^{z} \rho_{\beta}\right] \cos \left[\pi \Theta_{N}\left(\theta_{1}\right)+\pi \Theta_{N}\left(\theta_{2}\right)\right] \\
& +\operatorname{Tr}\left[\sigma_{i}^{y} \rho_{\beta}\right] \sin \left[\pi \Theta_{N}\left(\theta_{1}\right)+\pi \Theta_{N}\left(\theta_{2}\right)\right] \\
& +\left(\operatorname{Tr}\left[\mu_{i}^{\dagger} \rho_{\beta}\right]\left[\frac{2}{3} e^{i(2 \pi / 3) \Theta_{N}\left(\theta_{1}\right)}+\frac{1}{3} e^{-i(4 \pi / 3) \Theta_{N}\left(\theta_{1}\right)}\right]+\text { H.c. }\right) .
\end{aligned}
$$

We plot this observable in Fig. 6 using $\operatorname{Tr}\left[\sigma_{i}^{z} \rho_{\beta}\right]=0.9$ and $\operatorname{Tr}\left[\mu_{i} \rho_{\beta}\right]=\operatorname{Tr}\left[\mu_{i}^{\dagger} \rho_{\beta}\right]=0.8$.

\section{APPENDIX E: TOPOLOGICAL PHASES}

As we mentioned in Sec. V, the idea behind classifying quasiperiodic topological phases is to look at the nontrivial micromotion of the eigenstates. First, we make the following observation [108-115]: All the existing classifications of topological phases in $d$ spatial dimensions with unitary symmetry $G$ (for example, Refs. [119,125,145-147]) can be expressed in terms of classifying the homotopy classes of maps $B G \rightarrow \Theta_{d}$ for some space $\Theta_{d}$. Here, $B G$ is the classifying space for the group $G$, which is defined as $B G=E G / G$, where $E G$ is any contractible space on which $G$ acts freely ( $B G$ is unique up to homotopy equivalence). In the case that $\Theta$. forms a so-called " $\Omega$-spectrum," the preceding observation amounts to the assumption that the classification is given by some generalized cohomology theory evaluated on $B G$ [112,114], which is the case for all known classifications of invertible topological phases. However, our observation is more general than that, and it also applies to classifications of noninvertible phases, for example, the $G$-crossed modular tensor category classification of symmetry-enriched topological phases in two spatial dimensions $[109,125,148,149]$.

Now, in all these classifications, $\Theta_{d}$ is some abstract space that can be introduced in a formal mathematical way. However, what we want to posit is that this space actually has physical meaning. Specifically, we want to say $\Theta_{d}$ is actually homotopy equivalent to $\Omega_{d}$, the space of all gapped ground states, introduced in Sec. V. Indeed, Kitaev (Appendix F of Ref. [110]) has shown, at the microscopic level, that any $G$-symmetric gapped ground state in $d$ spatial dimensions gives rise to a map $B G \rightarrow \Omega_{d}$, which would explain the origins of the maps $B G \rightarrow \Theta_{d}$ described above.

If these conjectures hold, then it is clear how to classify maps $\mathcal{X} \rightarrow \Omega_{d}$ in the presence of symmetry $G$. First of all, we use the approach of Kitaev to turn this into a map $\mathcal{X} \times B G \rightarrow \Omega_{d}$, which is equivalent to a map $\mathcal{X} \times B G \rightarrow \Theta_{d}$. In other words, we simply replace $B G \rightarrow \mathcal{X} \times B G$ in the formulation of the classification.

We are now in a position to show how homotopy classes of maps $\mathbb{T}^{m} \rightarrow \Omega_{d}$ are related to SPT/SET phases with symmetry $\mathbb{Z}^{\times m}$. Indeed, this relation follows from the mathematical fact that

$$
B\left(\mathbb{Z}^{m}\right) \cong \mathbb{T}^{m},
$$

as can be seen by setting $E\left(\mathbb{Z}^{m}\right)=\mathbb{R}^{m}$, with $\mathbb{Z}^{m}$ acting freely by discrete translations. More generally, in the case of systems with symmetry (other than time-translation symmetry) $G$, we want to classify maps $\mathbb{T}^{m} \times B G \rightarrow \Omega_{d}$, and these are in one-to-one correspondence with topological phases with symmetry $G \times \mathbb{Z}^{m}$, as can be seen from the equation

$$
B\left(\mathbb{Z}^{m} \times G\right) \cong \mathbb{T}^{m} \times B G
$$

Now, let us consider cases where the states under consideration are invertible states; in other words, they do not admit fractionalized excitations such as anyons. In that case, as we have already mentioned, it is believed that the spaces $\Theta_{d}$ for different dimensions are related in a particular way. Specifically, the idea is that, if we define for any space $\mathcal{X}, h^{q}(\mathcal{X})$ to be the homotopy classes of maps $\mathcal{X} \rightarrow \Theta_{q}$, then $h^{\bullet}(-)$ should define a generalized cohomology theory, which obeys some set of axioms [150]. For any generalized cohomology theory, one can show, using the suspension and wedge axioms and the fact that [150] $\Sigma \mathbb{T}^{d}=\Sigma\left(S^{1} \vee \mathbb{T}^{m-1} \vee \Sigma \mathbb{T}^{m-1}\right)$ (here, $\Sigma$ denotes suspension and $\vee$ denotes wedge sum),

$$
h^{q}\left(\mathbb{T}^{m}\right)=h^{q}\left(S^{1}\right) \times h^{q}\left(\mathbb{T}^{m-1}\right) \times h^{q-1}\left(\mathbb{T}^{m-1}\right) .
$$

By applying this formula recursively, we derive Eq. (48).

As we have described, the emergent $\mathbb{Z}_{2} \times \mathbb{Z}_{2}$ symmetry arises as a symmetry of the effective static Hamiltonian in the twisted high-frequency limit. Nevertheless, we ultimately want to interpret the topological phases that we construct as protected by multiple time-translation symmetries, i.e., as being protected by $\mathbb{Z} \times \mathbb{Z}$. Naturally, any SPT protected via $\mathbb{Z}_{2} \times \mathbb{Z}_{2}$ can also be interpreted as a SPT protected by $\mathbb{Z} \times \mathbb{Z}$ since there is a homomorphism from $\mathbb{Z} \times \mathbb{Z} \rightarrow \mathbb{Z}_{2} \times \mathbb{Z}_{2}$. On the other hand, if we go beyond the twisted high-frequency limit (for example, if we assume 
that quasiperiodically driven systems can be MBL without needing to appeal to a twisted high-frequency limit), we can still apply the general analysis of Sec. VA, but it is not clear that the emergent $\mathbb{Z}_{2} \times \mathbb{Z}_{2}$ is expected to survive even if we remain within the same quasiperiodic topological phase. Therefore, the signatures of the phase that we discuss might need to be modified.

\section{APPENDIX F: SIGNATURES OF QUASIPERIODICALLY DRIVEN TOPOLOGICAL PHASES BEYOND THE TWISTED HIGH- FREQUENCY LIMIT}

As we mentioned in Sec. V B, it is not automatically clear that any signatures of a quasiperiodically driven topological phase that are based on the presence of the emergent finite internal symmetry group $\mathcal{G}$ will necessarily be robust in some stabilization of the phase that is beyond the twisted high-frequency limit. Let us discuss this situation in more detail in the phase that we discussed in Sec. V B, which had emergent symmetry $\mathcal{G}=\mathbb{Z}_{2} \times \mathbb{Z}_{2}$ and microscopic symmetry $G=\mathbb{Z}_{2}$.

The homomorphism $\mathbb{Z} \times \mathbb{Z} \times G \rightarrow \mathbb{Z}_{2} \times \mathbb{Z}_{2} \times \mathbb{Z}_{2}$ induces a corresponding pullback map $\mathcal{H}^{4}\left(\mathbb{Z}_{2} \times \mathbb{Z}_{2} \times \mathbb{Z}_{2}, \mathbb{Z}\right) \rightarrow$ $\mathcal{H}^{4}\left(\mathbb{Z} \times \mathbb{Z} \times \mathbb{Z}_{2}, \mathbb{Z}\right) \cong \mathcal{H}^{2}\left(\mathbb{Z}_{2}, \mathbb{Z}\right) \cong \mathcal{H}^{1}\left(\mathbb{Z}_{2}, \mathrm{U}(1)\right) \cong \mathbb{Z}_{2}$. The phase discussed in Sec. V B remains nontrivial under this pullback map. However, the signature of the projective representation of the emergent $\mathbb{Z}_{2} \times \mathbb{Z}_{2}$ on a symmetry flux of the microscopic $\mathbb{Z}_{2}$ is not robust. This is because the projective representation of the emergent symmetry corresponds to the nontrivial class in $\mathcal{H}^{3}\left(\mathbb{Z}_{2} \times \mathbb{Z}_{2}, \mathbb{Z}\right) \cong$ $\mathcal{H}^{2}\left(\mathbb{Z}_{2} \times \mathbb{Z}_{2}, \mathrm{U}(1)\right)$, and it maps to the trivial class under the pullback map $\mathcal{H}^{3}\left(\mathbb{Z}_{2} \times \mathbb{Z}_{2}, \mathbb{Z}\right) \rightarrow \mathcal{H}^{3}(\mathbb{Z} \times \mathbb{Z}, \mathbb{Z})$.

We can understand this case better if we imagine computing the $\mathcal{H}^{4}$ classifications using the Künneth formula. Viewed as a $\mathbb{Z}_{2}^{\times 3}$ SPT, our SPT lives in the factor

$$
\mathbb{Z}_{2} \cong \mathcal{H}^{1}\left(\mathbb{Z}_{2}, \mathcal{H}^{3}\left(\mathbb{Z}_{2} \times \mathbb{Z}_{2}, \mathbb{Z}\right)\right) \leq \mathcal{H}^{4}\left(\mathbb{Z}_{2}^{\times 3}, \mathbb{Z}\right),
$$

which precisely corresponds to the statement that a $\mathbb{Z}_{2}$ symmetry flux corresponds to a projective representation of $\mathbb{Z}_{2} \times \mathbb{Z}_{2}$. On the other hand, viewed as a $\mathbb{Z} \times \mathbb{Z} \times \mathbb{Z}_{2}$ SPT, our SPT lives in the factor

$$
\mathbb{Z}_{2} \cong \mathcal{H}^{2}\left(\mathbb{Z}_{2}, \mathcal{H}^{2}(\mathbb{Z} \times \mathbb{Z}, \mathbb{Z})\right) \leq \mathcal{H}^{4}\left(\mathbb{Z} \times \mathbb{Z} \times \mathbb{Z}_{2}, \mathbb{Z}\right) .
$$

In other words, relaxing the $\mathbb{Z}_{2} \times \mathbb{Z}_{2}$ symmetry to a $\mathbb{Z} \times \mathbb{Z}$ symmetry causes our phase to jump into a different factor of the Künneth formula, which in turn suggests that we should expect different physical signatures. Indeed, according to the usual physical interpretation of the Künneth formula, Eq. (F2) seems to show that if we annihilate two symmetry fluxes of the microscopic $\mathbb{Z}_{2}$ symmetry, then we leave behind a nontrivial zero-dimensional SPT classified by $\mathcal{H}^{2}(\mathbb{Z} \times \mathbb{Z}, \mathbb{Z}) \cong \mathbb{Z}$. The latter corresponds to quantized energy pumping between the two incommensurate frequencies (see Sec. VC), so we can interpret this case as the statement that a symmetry flux of the microscopic $\mathbb{Z}_{2}$ symmetry carries a half-quantized frequency pump. We leave a more systematic understanding of this effect to future work.

\section{APPENDIX G: RARITY OF RESONANCES IN QUASIPERIODICALLY DRIVEN SYSTEMS}

The crucial mathematical property of quasiperiodic driving that we employ in our paper is that, although for any given frequency vector $\boldsymbol{\omega}=\left(\omega_{1}, \ldots, \omega_{m}\right)$ the spectrum of possible multiphoton emission and absorption processes (i.e., $\boldsymbol{\omega} \cdot \boldsymbol{n}$ for integer vectors $\boldsymbol{n}$ ) is dense everywhere (and, in particular, at zero), if we restrict ourselves to low-order processes (i.e., $|\boldsymbol{n}|$ small), the possible values of $|\boldsymbol{\omega} \cdot \boldsymbol{n}|$ are still somewhat sparse. To quantify this, let us introduce the following definition.

Definition 1. Let $\omega \in \mathbb{R}^{n}$ be a nonzero vector. Then, the exponent $\sigma(\boldsymbol{\omega})$ is the smallest number $\sigma$ such that for all $\epsilon>0$, there exists a constant $c>0$ (depending on $\omega$ and $\epsilon$ ) such that

$$
|\boldsymbol{\omega} \cdot \boldsymbol{n}| \geq \frac{c|\boldsymbol{\omega}|}{|\boldsymbol{n}|^{\sigma+\epsilon}}
$$

for all nonzero integer vectors $|\boldsymbol{n}|$.

Note that this exponent is invariant under rescaling the frequencies, i.e., $\sigma(a \boldsymbol{\omega})=\sigma(\boldsymbol{\omega})$ for any real $a \neq 0$.

Clearly, if the frequencies are rationally related, i.e., if there exists a nonzero integer vector $\boldsymbol{n}$ such that $\boldsymbol{n} \cdot \boldsymbol{\omega}=0$, then $\sigma(\boldsymbol{\omega})=\infty$. On the other hand, we have the lower bound given in Proposition 1 .

Proposition 1. For any $\omega \in \mathbb{R}^{m}, \sigma(\omega) \geq m-1$.

Proof.-This proposition is a higher-dimensional version of the famous Dirichlet approximation theorem [128]. Like that theorem, it can be proven using Minkowski's theorem [128], which states that if $C$ is some bounded convex subset of $\mathbb{R}^{m}$, whose volume satisfies $\mu(C)>2^{m}$, then $C \cap \mathbb{Z}^{m}$ contains at least one point other than 0 .

Indeed, using the rescaling invariance of $\sigma$ to set $|\omega|=1$, let us define $C_{R, c}=\left\{\boldsymbol{x} \in \mathbb{R}^{m}:|\boldsymbol{x} \cdot \boldsymbol{\omega}| \leq c,|\boldsymbol{x}| \leq R\right\}$; then, $\mu\left(C_{R, c}\right) \geq c v_{m} R^{m-1}$ for some constant $v_{m}$, which only depends on $m$. Hence, we can satisfy the condition to apply Minkowski's theorem to $C_{R, c}$ provided that $c v_{m} R^{m-1}>2^{m}$, which means that for any $R>0$, there exists $\boldsymbol{n} \in \mathbb{Z}^{d}$ nonzero with $|\boldsymbol{n}| \leq R$ such that $|\boldsymbol{\omega} \cdot \boldsymbol{n}| \leq 2^{m+1} v_{m}^{-1} R^{-(m-1)}$. This result indeed implies that we must have $\sigma(\boldsymbol{\omega}) \geq m-1$.

In fact, this lower bound is generically saturated, in the following precise sense.

Proposition 2. Let $W$ be the set of all $\boldsymbol{\omega} \in \mathbb{R}^{m}$ such that $\sigma(\boldsymbol{\omega})>m-1$. Then, we have the Lebesgue measure $\mu(W)=0$. In other words, almost all $\omega$ 's have $\sigma(\boldsymbol{\omega})=m-1$. 
Proof.-We extend the proof given for the case $m=2$ in Ref. [151]. By the rescaling invariance of $\sigma(\boldsymbol{\omega})$, it is sufficient to prove that $\mu(S)=0$, where $S$ is the intersection of $W$ with $B$, the unit ball in $\mathbb{R}^{m}$.

Define $S_{n, c, \gamma}$ to be the set of all vectors $\omega \in B$ such that

$$
|\boldsymbol{\omega} \cdot \boldsymbol{n}|<c|\boldsymbol{n}|^{-\gamma}
$$

Observe that $\mu\left(S_{\boldsymbol{n}, c, \gamma}\right) \leq c u_{m}|\boldsymbol{n}|^{-(\gamma+1)}$, for some constant $u_{m}$ that only depends on $m$. Now, define $S_{\boldsymbol{c}, \gamma}=\cup_{\boldsymbol{n} \in \mathbb{Z}^{m}} S_{\boldsymbol{n}, \boldsymbol{c}, \gamma}$. Then, we see that

$$
\mu\left(S_{c, \gamma}\right) \leq c u_{m} \sum_{n \in \mathbb{Z}^{m}}|\boldsymbol{n}|^{-(\gamma+1)}:=c \Sigma_{\gamma}<\infty,
$$

provided that $\gamma>m-1$. Next, define $S_{\gamma}=\mathrm{\cap}_{c>0} S_{c, \gamma}$. We see that $\mu\left(S_{\gamma}\right) \leq c \Sigma_{\gamma}$ for all $c>0$, from which we conclude that $\mu\left(S_{\gamma}\right)=0$. Finally, since we can write $S=\cup_{\gamma>m-1} S_{\gamma}$, we conclude that $\mu(S)=0$.

Finally, let us give a concrete example of a frequency vector $\boldsymbol{\omega}$ with $\sigma(\boldsymbol{\omega})=m-1$. Indeed, we have the following proposition.

Proposition 3. Let $\omega$ be a vector of frequencies that are not rationally related, and suppose that all ratios $\omega_{i} / \omega_{j}$ are algebraic numbers; i.e., each one is a root of some polynomial equation with integer coefficients. Then, $\sigma(\boldsymbol{\omega})=m-1$.

Proof.-This proposition is known as the subspace theorem $[127,128]$.

\section{APPENDIX H: PROOF OF LONG-LIVED PREHEATING REGIME AND EMERGENT SYMMETRIES FROM TWISTED TIME-TRANSLATION SYMMETRIES}

In this section, we provide the full, rigorous formulation of our statements on a long-lived, preheating dynamical description of quasiperiodically driven systems. Our starting point is the iterative procedure described in Sec. VII, which we carry out up to some optimal iteration order $q_{*}$, to be computed. Here, we develop the technical machinery needed to prove rigorous bounds on this iteration and thereby prove our theorem.

\section{Some definitions}

We adopt the notion of a potential from Ref. [34], which is a formal sum

$$
\Phi=\sum_{Z} \Phi_{Z}
$$

where the sum is over subsets $Z$ of the lattice. In order to analyze quasiperiodic driving, we want to consider potentials that are parametrized by the torus $\mathbb{T}^{m}$, that is, $\Phi(\boldsymbol{\theta})$ where $\boldsymbol{\theta} \in \mathbb{T}^{m}$.
It is convenient to analyze the $\boldsymbol{\theta}$ dependence in Fourier space. Accordingly, we define a "colored potential" to be a formal sum

$$
\Phi(\boldsymbol{\theta})=\sum_{Z, \boldsymbol{n}} \Phi_{Z, \boldsymbol{n}} e^{i \boldsymbol{\theta} \cdot \boldsymbol{n}}
$$

where $\boldsymbol{n} \in \mathbb{Z}^{m}$ represents a Fourier mode.

We can define the formal commutator of colored potentials according to

$$
[\Phi(\boldsymbol{\theta}), \Theta(\boldsymbol{\theta})]=\sum_{Z, \boldsymbol{n}, Z^{\prime}, \boldsymbol{n}^{\prime}}\left[\Phi_{Z, \boldsymbol{n}}, \Theta_{Z^{\prime}, \boldsymbol{n}^{\prime}}\right] e^{i \boldsymbol{\theta} \cdot\left(\boldsymbol{n}+\boldsymbol{n}^{\prime}\right)},
$$

where we take the commutator on the right-hand side to be supported on $Z \cup Z^{\prime}$ whenever $Z$ and $Z^{\prime}$ are nondisjoint (otherwise the commutator is zero). In other words,

$$
[\Phi, \Theta]_{Z, \boldsymbol{n}}=\sum_{\substack{Z_{1}, Z_{2}: Z_{1} \cup Z_{2}=Z_{2} \\ Z_{1} \text { and } Z_{2} \text { nondisisoint }}} \sum_{n_{1}, \boldsymbol{n}_{2}: \boldsymbol{n}_{1}+\boldsymbol{n}_{2}=\boldsymbol{n}}\left[\Phi_{Z_{1}, \boldsymbol{n}_{1}}, \Theta_{Z_{2}, \boldsymbol{n}_{2}}\right] .
$$

We can simplify our notation by defining a "colored set" to be a pair $\boldsymbol{Z}=(\boldsymbol{Z}, \boldsymbol{n})$. We define a " $U$ " operator on colored sets according to $\left(Z_{1}, \boldsymbol{n}_{1}\right) \cup\left(Z_{2}, \boldsymbol{n}_{2}\right)=\left(Z_{1} \cup Z_{2}, \boldsymbol{n}_{1}+\boldsymbol{n}_{2}\right)$, and we declare that two colored sets $\left(Z_{1}, \boldsymbol{n}_{1}\right)$ and $\left(Z_{2}, \boldsymbol{n}_{2}\right)$ are "disjoint" if and only if $Z_{1}$ and $Z_{2}$ are disjoint. Then, Eq. (H4) simply becomes

$$
[\Phi, \Theta]_{Z}=\sum_{\substack{Z_{1}, Z Z_{2}: Z_{1} \cup Z_{2}=Z \\ Z_{1} \text { and } Z_{2} \text { nondisjoint }}}\left[\Phi_{Z_{1}}, \Theta_{Z_{2}}\right],
$$

which is formally identical to the uncolored case. As in the uncolored case, we define the exponentiated action of one potential $\Theta$ on another $\Phi$ according to

$$
e^{\Theta} \Phi e^{-\Theta}:=\sum_{n=0}^{\infty} \frac{1}{n !} \operatorname{ad}_{\Theta}^{n} \Phi,
$$

where $\operatorname{ad}_{\Theta} \Phi:=[\Theta, \Phi]$.

We also can similarly write the norm Eq. (62) in this succinct notation as

$$
\|\Phi\|_{\kappa}=\sup _{x} \sum_{Z \ni x} e^{\kappa|Z|}\left\|\Phi_{Z}\right\|,
$$

where the supremum is over sites $x$ on the lattice and where we made the following (purely formal) definitions: $|(Z, \boldsymbol{n})|=|Z|+|\boldsymbol{n}|$, and $x \in(Z, \boldsymbol{n})$ if and only if $x \in Z$.

\section{Statement of the theorem}

Our starting point is the iterative procedure described in Sec. VII C. We can perform this iterative procedure formally at the level of colored potentials. The goal is to prove bounds on the iteration, which are encapsulated in 
the following theorem. We start the iteration from some colored potential $H^{(0)}(\boldsymbol{\theta})=D^{(0)}+V^{(0)}(\boldsymbol{\theta})$, where $D^{(0)}$ is constant (that is, $D_{Z, \boldsymbol{n}}^{(0)}=0$ for $\boldsymbol{n} \neq \mathbf{0}$ ) and $V^{(0)}(\boldsymbol{\theta})$ has a zero constant component (that is, $V_{Z, \mathbf{0}}^{(0)}=0$ ). We perform the iteration described in Sec. VII C, giving, at the $q$ th iteration, a colored potential $H^{(q)}(\boldsymbol{\theta})=D^{(q)}+V^{(q)}(\boldsymbol{\theta})$, where $D^{(q)}$ is constant and $V^{(q)}(\boldsymbol{\theta})$ has zero average on $\mathbb{T}^{m}$. Then, the theorem is as follows.

Theorem 1. Suppose that the driving frequency vector $\boldsymbol{\omega}$ obeys a Diophantine condition $|\boldsymbol{n} \cdot \boldsymbol{\omega}| \leq c|\boldsymbol{n}|^{-\gamma}|\boldsymbol{\omega}|$ for all integer vectors $\boldsymbol{n}$ where $\gamma>m-1$, and $c$ is a constant depending on the ratios $\omega_{i} / \omega_{j}$ but not on the overall scale of $\omega=|\omega|$.

We also assume that the norm of the driving frequency $\omega$ is large enough compared to some local energy scale $\omega_{0}$; namely, there exists a decay rate $\kappa_{0}>0$, such that

$$
\omega \geq K \omega_{0}, \quad q_{*} \geq 1,
$$

where

$$
q_{*}:=\left\lfloor K^{\prime}\left(\frac{\omega}{\omega_{0}}\right)^{\mu}\right\rfloor
$$

is the maximum iteration order. Here,

$$
\omega_{0}:=\max \left\{\left\|D^{(0)}\right\|_{\kappa_{0}},\left\|V^{(0)}\right\|_{\kappa_{0}}\right\}, \quad \mu=\frac{1}{\gamma+1},
$$

where the definition of the norm $\|\cdot\|_{\kappa_{0}}$ is given in Eq. (H7), and $K, K^{\prime}$ numerical constants, which do not depend on $D^{(0)}, V^{(0)}$, the geometry of the lattice, or the driving frequencies, are given by

$K=\frac{2^{2+\gamma}}{\kappa_{0}^{2+\gamma}} a, \quad K^{\prime}=2^{-4-2 \mu+\frac{1}{1+\mu}}(\mu+1)^{-1} \kappa_{0}^{\mu+1} a^{-\mu}$,

with $a=c^{-1} \gamma^{\gamma} e^{-\gamma} \times \max \left\{9 \times 2^{6+\gamma}, 3 \times 2^{1+\gamma} \kappa_{0}\right\}$.

At the $q_{*}$ th iteration, define $D:=D^{\left(q_{*}\right)}$ and $V:=V^{\left(q_{*}\right)}$. Then, we have

$$
\begin{gathered}
\left\|V^{\left(q_{*}\right)}\right\|_{\kappa_{q_{*}}} \leq \omega_{0}\left(\frac{1}{2}\right)^{q_{*}}, \\
\left\|D^{\left(q_{*}\right)}\right\|_{\kappa_{q_{*}}} \leq 2 \omega_{0}, \\
\left\|D^{\left(q_{*}\right)}-D^{(0)}\right\|_{\kappa_{q_{*}}} \leq C \omega_{0}\left(\frac{\omega_{0}}{\omega}\right),
\end{gathered}
$$

for some numerical constant $C$. Here, the decay constant $\kappa_{q_{*}}$ is given by $\kappa_{q_{*}}=\left(\kappa_{0} / 2\right)^{\mu+1}-(\mu+1)\left(a 2^{1 /(\mu+1)}\right)^{\mu} \lambda^{\mu}\left(q_{*}-1\right) \geq$ $\kappa_{0} / 4$. Additionally, for any potential $Z$, we have

$$
\left\|e^{\mathrm{ad}_{A}\left(q_{*}-1\right)} \cdots e^{\mathrm{ad}_{A^{(0)}}} Z-Z\right\|_{\kappa_{q_{*}}} \leq C\|Z\|_{\kappa_{0}}\left(\omega_{0} / \omega\right) .
$$

Note that in all bounds involving the decay constant $\kappa_{q_{*}}$, we can simply replace it by $\kappa_{0} / 4$. Also, we can identify the local energy scale $J$ in the main text as $\omega_{0}$. Then, Theorem 1 immediately yields Eqs. (65) and (66) in Sec. VII B of the main text. To prove Eq. (71), one invokes Lieb-Robinson bounds, following an identical argument as in Appendix D of Ref. [130] (but using the Lieb-Robinson bound for shortrange interactions). Equation (68) follows immediately from the arguments in Ref. [34] and our results above.

\section{The proof}

Our key technical tool is the following:

Lemma 1. Let $\Theta, \Phi$ be colored potentials and assume that $3\|\Theta\|_{\kappa} \leq \kappa-\kappa^{\prime}$, with $0<\kappa^{\prime}<\kappa$. Then,

$$
\left\|e^{\Theta} \Phi e^{-\Theta}-\Phi\right\|_{\kappa^{\prime}} \leq \frac{18}{\left(\kappa-\kappa^{\prime}\right) \kappa^{\prime}}\|\Theta\|_{\kappa}\|\Phi\|_{\kappa}
$$

and

$$
\left\|e^{\Theta} \Phi e^{-\Theta}\right\|_{\kappa^{\prime}} \leq\left(1+\frac{18}{\left(\kappa-\kappa^{\prime}\right) \kappa^{\prime}}\|\Theta\|_{\kappa}\right)\|\Phi\|_{\kappa^{\prime}} .
$$

Proof.-This lemma is the colored potential version of Lemma 4.1 from Ref. [34], and the proof follows line-byline identically to the proof of that lemma.

Armed with this lemma, we can now proceed to analyze the iteration in a similar manner to what Ref. [34] did for the periodic case. The key new aspect compared to the periodic case is that the formula for $A^{(q)}$ in terms of $V^{(q)}$, Eq. (80), involves a denominator that could potentially become very small. However, the Diophantine condition assumption in the theorem allows us to bound this denominator, which gives the following lemma.

Lemma 2. For $0<\kappa^{\prime}<\kappa$, and $\omega$ obeying the Diophantine condition $|\boldsymbol{n} \cdot \boldsymbol{\omega}| \leq c|\boldsymbol{n}|^{-\gamma}|\boldsymbol{\omega}|$ with $\gamma>m-1$ for all integer vectors $\boldsymbol{n} \in \mathbb{Z}^{m}$ and a constant $c$, we have

$$
\left\|A^{(q)}\right\|_{\kappa^{\prime}} \leq \frac{c^{\prime}}{\left(\kappa-\kappa^{\prime}\right)^{\gamma}} \frac{\left\|V^{(q)}\right\|_{\kappa}}{\omega},
$$

where $c^{\prime}=c^{-1} \gamma^{\gamma} e^{-\gamma}$.

Proof.-

$$
\begin{aligned}
\left\|A^{(q)}\right\|_{\kappa^{\prime}} & =\sup _{x} \sum_{Z \ni x, \boldsymbol{n}} e^{\kappa^{\prime}(|Z|+|\boldsymbol{n}|)}\left\|A_{Z, \boldsymbol{n}}^{(q)}\right\| \\
& =\sup _{x} \sum_{Z \ni x, \boldsymbol{n}} e^{\kappa(|Z|+|\boldsymbol{n}|)} \frac{1}{|\boldsymbol{\omega} \cdot \boldsymbol{n}|}\left\|V_{Z, \boldsymbol{n}}^{(q)}\right\| \\
& \leq \frac{\omega^{-1}}{c} \sup _{x} \sum_{Z \ni x, \boldsymbol{n}} e^{\kappa^{\prime}(|Z|+|\boldsymbol{n}|)}|\boldsymbol{n}|^{\gamma}\left\|V_{Z, \boldsymbol{n}}^{(q)}\right\| \\
& \leq \frac{c^{\prime} \omega^{-1}}{\left(\kappa-\kappa^{\prime}\right)^{\gamma}} \sup _{x} \sum_{Z \ni x, \boldsymbol{n}} e^{\kappa^{\prime}(|Z|+|\boldsymbol{n}|)} e^{\left(\kappa-\kappa^{\prime}\right)|\boldsymbol{n}|}\left\|V_{Z, \boldsymbol{n}}^{(q)}\right\| \\
& \leq \frac{c^{\prime}}{\left(\kappa-\kappa^{\prime}\right)^{\gamma}} \frac{\left\|V^{(q)}\right\|_{\kappa}}{\omega},
\end{aligned}
$$


and we use the inequality

$$
y^{\gamma} e^{-\epsilon y} \leq(\gamma / \epsilon)^{\gamma} e^{-\gamma}
$$

for $y, \epsilon>0$.

Now, we proceed as in Ref. [34]. Specifically, we introduce

$$
\gamma_{q}(O)=e^{-A^{(q)}} O e^{A^{(q)}}
$$

and

$$
\alpha_{q}(O)=\int_{0}^{1} d s e^{-s A^{(q)}} O e^{s A^{(q)}}
$$

the latter of which satisfies $e^{-A^{(q)}}\left(\boldsymbol{\omega} \cdot \partial_{\boldsymbol{\theta}}\right) e^{A^{(q)}}=\alpha_{q}\left(\boldsymbol{\omega} \cdot \partial_{\boldsymbol{\theta}} A^{(q)}\right)$. Then, we see that

$$
H^{(q+1)}=\gamma_{q}\left(D^{(q)}\right)+\left(\gamma_{q}\left(V^{(q)}\right)-V^{(q)}\right)+\left(\alpha_{q}\left(V^{(q)}\right)-V^{(q)}\right),
$$

from which we obtain

$$
\begin{aligned}
& D^{(q+1)}=D^{(q)}+\left\langle W^{(q)}\right\rangle, \\
& V^{(q+1)}=W^{(q)}-\left\langle W^{(q)}\right\rangle
\end{aligned}
$$

[recalling that $\langle\cdot\rangle$ is the time-averaging operation $\langle O\rangle:=$ $\left.\left[\int d^{m} \boldsymbol{\theta} /(2 \pi)^{m}\right] O(\boldsymbol{\theta})=O_{0}\right]$, where

$$
\begin{aligned}
W^{(q)}=\left(\gamma_{q}\left(D^{(q)}\right)-D^{(q)}\right)+ & \left(\gamma_{q}\left(V^{(q)}\right)-V^{(q)}\right) \\
& +\left(\alpha_{q}\left(V^{(q)}\right)-V^{(q)}\right) .
\end{aligned}
$$

Let us suppose there is a sequence of strictly decreasing decay constants $\kappa_{0}>\kappa_{1}>\kappa_{2}>\cdots>0$ (we define this sequence later); we then have

$$
\begin{gathered}
\left\|D^{(q+1)}\right\|_{\kappa_{q+1}} \leq\left\|D^{(q)}\right\|_{\kappa_{q}}+w_{q} / 2, \\
\left\|V^{(q+1)}\right\|_{\kappa_{q+1}} \leq w_{q}, \\
\left\|D^{(q+1)}-D^{(q)}\right\|_{\kappa_{q+1}} \leq w_{q} / 2,
\end{gathered}
$$

where $w_{q}=2\left\|W^{(q)}\right\|_{\kappa_{q+1}}$. Using Lemmas 1 and 2, we see that, provided

$$
\frac{3 c^{\prime}}{\left(\kappa_{q}-\kappa_{q}^{\prime}\right)^{\gamma}} \frac{\left\|V^{(q)}\right\|_{\kappa_{q}}}{\omega} \leq \kappa_{q}^{\prime}-\kappa_{q+1},
$$

we obtain

$$
w_{q} \leq \frac{36 c^{\prime}\left(\left\|D^{(q)}\right\|_{\kappa^{(q)}}+2\left\|V^{(q)}\right\|_{\kappa_{q}}\right)}{\kappa_{q+1}\left(\kappa_{q}^{\prime}-\kappa_{q+1}\right)\left(\kappa_{q}-\kappa_{q}^{\prime}\right)^{\gamma}} \frac{\left\|V^{(q)}\right\|_{\kappa_{q}}}{\omega},
$$

where we have chosen $0<\kappa_{q+1}<\kappa_{q}^{\prime}<\kappa_{q}$. In particular, we choose to let $\kappa_{q}^{\prime}=\left(\kappa_{q}+\kappa_{q+1}\right) / 2$, so we obtain

$w_{q} \leq \frac{\left(36 \times 2^{1+\gamma)} c^{\prime}\left(\left\|D^{(q)}\right\|_{\kappa_{q}}+2\left\|V^{(q)}\right\|_{\kappa_{q}}\right)\right.}{\kappa_{q+1}\left(\kappa_{q}-\kappa_{q+1}\right)^{\gamma+1}} \frac{\left\|V^{(q)}\right\|_{\kappa_{q}}}{\omega}$,

while the requirement Eq. (H30) becomes

$$
\left(3 \times 2^{1+\gamma}\right) c^{\prime} \frac{\left\|V^{(q)}\right\|_{\kappa_{q}}}{\omega} \leq\left(\kappa_{q}-\kappa_{q+1}\right)^{\gamma+1} .
$$

Now, we turn to the question of how we should choose the decay constants $\kappa_{q}$. A good way to do this is as follows (generalizing the approach of Ref. [130], which was an improvement on the original analysis of Ref. [34]). For notational brevity in what follows, we define $\lambda=\omega_{0} / \omega$, the small parameter.

First, let us make the induction hypothesis that for some $q,\left\|D^{(q)}\right\|_{\kappa_{q}} \leq 2 \omega_{0},\left\|V^{(q)}\right\|_{\kappa_{q}} \leq \omega_{0}(1 / 2)^{q}$. What is the choice of $\kappa_{q+1}$ that ensures we also have $\left\|V_{q+1}\right\|_{\kappa_{q+1}} \leq \omega_{0}(1 / 2)^{q+1}$ along with Eq. (H33)? Indeed, this result is ensured provided that

$$
\frac{a \lambda}{\kappa_{q+1}\left(\kappa_{q}-\kappa_{q+1}\right)^{\gamma+1}} \leq 1
$$

with $a=c^{\prime} \max \left\{C_{1}, C_{2} \kappa_{0}\right\}$, where $C_{1}=\left(144 \times 2^{2+\gamma}\right)$ and $C_{2}=\left(3 \times 2^{1+\gamma}\right)$. We would also like for $w_{0}$ to be "small" in the factor $1 / \omega$; this idea suggests that we should set the difference $\kappa_{0}-\kappa_{1}$ to be independent of $\omega$. We achieve this result by letting

$$
\kappa_{0} / \kappa_{1}=2 .
$$

The condition, Eq. (H34), that needs to be satisfied for $q=0$ then reads

$$
\lambda \leq \frac{\kappa_{0}^{2+\gamma}}{2^{2+\gamma}} \frac{1}{a} .
$$

To choose the subsequent constants $\kappa_{q \geq 2}$, intuitively, we can imagine that, for small $\lambda, \kappa_{q \geq 1}$ varies very slowly as a function of $n$; thus, we can treat $q$ as a continuous variable so that $\kappa_{q} \mapsto k(q)$, and Eq. (H34) becomes

$$
\frac{a \lambda}{k(q)(-d k(q) / d q)^{\gamma+1}} \leq 1 .
$$

If we choose Eq. (H37) to be saturated, we obtain the differential equation

$$
\frac{d k(q)}{d q}=-a^{\mu}\left(\frac{\lambda}{k(q)}\right)^{\mu}, \quad \mu=\frac{1}{\gamma+1}
$$

with the solution 


$$
k(q)^{\mu+1}=k(1)^{\mu+1}-(\mu+1) a^{\mu} \lambda^{\mu}(q-1) .
$$

Armed with this intuition, we define the decay rates $\kappa_{q}$ that we employ as the values obtained from evaluating the following generalized function $\kappa(q)$ at integer values $q \geq 1$ :

$$
\begin{aligned}
\kappa_{q} & :=\kappa(q) \text { for } q=1,2,3, \ldots, \text { where } \\
\kappa(q)^{\mu+1} & :=\kappa_{1}^{\mu+1}-(\mu+1) b^{\mu} \lambda^{\mu}(q-1) \text { for } q \in \mathbb{R} .
\end{aligned}
$$

We remark that the decay rate is only physically meaningful and mathematically useful when $\kappa_{q}>0$, and we will find conditions later that restrict us to this range. Note also the different constant $b$ from $a$ of Eq. (H39), which will allow us to correct for the fact that we actually want to satisfy the finite difference equation (H34) rather than the differential equation (H37). We then find that $\kappa(q)$ satisfies the differential equation with $a$ replaced by $b$, i.e.,

$$
\frac{d \kappa}{d q}=-b^{\mu}\left(\frac{\lambda}{\kappa}\right)^{\mu}, \quad \mu=\frac{1}{\gamma+1} .
$$

Since $\kappa(q)$ is a concave down function, we have that

$$
\kappa_{q}-\kappa_{q+1} \geq-\kappa^{\prime}(q)=b^{\mu}\left(\lambda / \kappa_{q}\right)^{\mu},
$$

and moreover,

$\left(\kappa_{q+1} / \kappa_{q}\right)^{\mu+1}=1-\frac{(\mu+1) b^{\mu} \lambda^{\mu}}{\kappa_{q}^{\mu+1}} \geq 1-\frac{2^{\mu+1}(\mu+1) b^{\mu} \lambda^{\mu}}{\kappa_{1}^{\mu+1}}$,

assuming that we continue the iteration only while $\kappa_{q} \geq \kappa_{1} / 2=\kappa_{0} / 4$. Therefore, so long as we impose the condition

$$
\frac{2^{\mu+1}(\mu+1) b^{\mu} \lambda^{\mu}}{\kappa_{1}^{\mu+1}} \leq 1 / 2,
$$

then we have

$$
\left(\kappa_{q+1} / \kappa_{q}\right)^{\mu+1} \geq 1 / 2 .
$$

Combining Eqs. (H42) and (H45), we obtain

$$
\frac{b \lambda}{\kappa_{q+1}\left(\kappa_{q}-\kappa_{q+1}\right)^{\gamma+1}} \leq 2^{1 /(\mu+1)}
$$

and hence ensure that Eq. (H34) is satisfied, if we choose $b=a 2^{1 /(\mu+1)}$.

Thus, if we define the maximum iteration order $q^{*}$ as

$$
q_{*}:=\left\lfloor 2^{-4-2 \mu+\frac{1}{1+\mu}}(\mu+1)^{-1} \kappa_{0}^{\mu+1} a^{-\mu} \lambda^{-\mu}\right\rfloor
$$

and we demand that $\lambda$ (the parameter relating the local energy scale to the driving frequency) is small enough so that

$$
q_{*} \geq 1,
$$

(otherwise the theorem is trivial anyway), then Eq. (H44) is automatically satisfied.

Note also that the definition of the maximum iteration order $q_{*}$ indeed ensures that the iteration continues only while $\kappa_{q}>0$ [in fact, $\kappa_{q}^{\mu+1} \geq(3 / 4) \kappa_{1}^{\mu+1}$ for $q \leq q_{*}$, so, in particular, $\kappa_{q} \geq \kappa_{1} / 2=\kappa_{0} / 4$ in agreement with our assumption).

To ensure that the induction hypothesis on $\left\|D^{(q)}\right\|_{\kappa_{q}}$ remains satisfied, let us note that Eq. (H34) also ensures that

$$
\left\|D^{(q+1)}\right\|_{\kappa_{q+1}} \leq\left\|D^{(q)}\right\|_{\kappa_{q}}+\frac{1}{2} \omega_{0}\left(\frac{1}{2}\right)^{q},
$$

and summing over $q$ ensures $\left\|D^{(q)}\right\|_{\kappa_{q}} \leq 2 \omega_{0}$.

Let us now prove the bounds on $\left\|D^{\left(q_{*}\right)}-D^{(0)}\right\|_{\kappa_{q_{*}}}$ and on the potential. We first use that

$$
\begin{aligned}
\left\|D^{(q+1)}-D^{(q)}\right\|_{\kappa_{q_{*}}} & \leq\left\|D^{(q+1)}-D^{(q)}\right\|_{\kappa_{q}} \leq \frac{1}{2}\left(\frac{1}{2}\right)^{q} w_{0} \\
& \leq C e^{-c q} \omega_{0}\left(\frac{\omega_{0}}{\omega}\right) .
\end{aligned}
$$

Summing the lhs up to $q_{*}$ then gives the desired result, redefining the constant $C$.

Next,

$$
\begin{aligned}
\left\|e^{\mathrm{ad}_{A}(q)} \cdots e^{\mathrm{ad}_{A^{(0)}}} Z\right\|_{\kappa_{q+1}} & \leq\|Z\|_{\kappa_{0}} \prod_{j=0}^{q}\left(1+C\left(\frac{\omega_{0}}{\omega}\right) e^{-c j}\right) \\
& \leq C\|Z\|_{\kappa_{0}},
\end{aligned}
$$

with redefined $C$, using Lemmas 1 and 2, and

$$
\begin{aligned}
& \left(1+\frac{2^{1+\gamma} \times 18 c^{\prime}}{\kappa_{q+1}\left(\kappa_{q-1}-\kappa_{q}\right)^{1+\gamma}} \frac{\left\|V^{(q)}\right\|_{\kappa_{q}}}{\omega}\right) \\
& \leq\left(1+C^{\prime} \frac{\kappa_{q}}{\kappa_{q+1}} \frac{\left\|V^{(q)}\right\|_{\kappa_{q}}}{b \lambda \omega}\right) \\
& \leq\left(1+C\left(\omega_{0} / \omega\right) e^{-c q}\right),
\end{aligned}
$$

with $\left(\kappa_{q} / \kappa_{q+1}\right)<2^{1 /(\mu+1)}$ and $\left\|V^{(q)}\right\|_{\kappa_{q}} \leq w_{0}(1 / 2)^{q-1} \leq$ $d e^{-c q} \omega_{0}\left(\omega_{0} / \omega\right)$.

Then, repeating the proof of Theorem 2.1 in Ref. [34], defining

$$
E_{\kappa_{q+1}}:=e^{\operatorname{ad}_{A}(q)} \cdots e^{\operatorname{ad}_{A^{(0)}}} Z-Z,
$$


we have

$$
\left\|E_{\kappa_{q+1}}-E_{\kappa_{q}}\right\| \leq C\left(\omega_{0} / \omega\right) e^{-c q}
$$

which after summing yields

$$
\begin{aligned}
\left\|e^{\operatorname{ad}_{A}\left(q_{*}-1\right)} \cdots e^{\operatorname{ad}_{A^{(0)}}} Z-Z\right\|_{\kappa_{q_{*}}} & =\left\|E_{\kappa_{q_{*}}}\right\|_{\kappa_{q_{*}}} \\
& \leq C\|Z\|_{\kappa_{0}}\left(\omega_{0} / \omega\right) .
\end{aligned}
$$

This concludes the proof of the theorem.

[1] M.Z. Hasan and C. L. Kane, Colloquium: Topological Insulators, Rev. Mod. Phys. 82, 3045 (2010).

[2] X.-L. Qi and S.-C. Zhang, Topological Insulators and Superconductors, Rev. Mod. Phys. 83, 1057 (2011).

[3] T. Senthil, Symmetry-Protected Topological Phases of Quantum Matter, Annu. Rev. Condens. Matter Phys. 6, 299 (2015).

[4] X.-G. Wen, Colloquium: Zoo of Quantum-Topological Phases of Matter, Rev. Mod. Phys. 89, 041004 (2017).

[5] A. Eckardt, Colloquium: Atomic Quantum Gases in Periodically Driven Optical Lattices, Rev. Mod. Phys. 89, 011004 (2017).

[6] R. Moessner and S. L. Sondhi, Equilibration and Order in Quantum Floquet Matter, Nat. Phys. 13, 424 (2017).

[7] D. V. Else, C. Monroe, C. Nayak, and N. Y. Yao, Discrete Time Crystals, arXiv:1905.13232.

[8] F. Harper, R. Roy, M. S. Rudner, and S. L. Sondhi, Topology and Broken Symmetry in Floquet Systems, arXiv:1905. 01317.

[9] V. Khemani, A. Lazarides, R. Moessner, and S. L. Sondhi, Phase Structure of Driven Quantum Systems, Phys. Rev. Lett. 116, 250401 (2016).

[10] C. W. von Keyserlingk and S. L. Sondhi, Phase Structure of One-Dimensional Interacting Floquet Systems. II. Symmetry-Broken Phases, Phys. Rev. B 93, 245146 (2016).

[11] D. V. Else, B. Bauer, and C. Nayak, Floquet Time Crystals, Phys. Rev. Lett. 117, 090402 (2016).

[12] D. V. Else, B. Bauer, and C. Nayak, Prethermal Phases of Matter Protected by Time-Translation Symmetry, Phys. Rev. X 7, 011026 (2017).

[13] N. Y. Yao, A. C. Potter, I.-D. Potirniche, and A. Vishwanath, Discrete Time Crystals: Rigidity, Criticality, and Realizations, Phys. Rev. Lett. 118, 030401 (2017).

[14] W. W. Ho, S. Choi, M. D. Lukin, and D. A. Abanin, Critical Time Crystals in Dipolar Systems, Phys. Rev. Lett. 119, 010602 (2017).

[15] J. Zhang, P. W. Hess, A. Kyprianidis, P. Becker, A. Lee, J. Smith, G. Pagano, I.-D. Potirniche, A. C. Potter, A. Vishwanath, N. Y. Yao, and C. Monroe, Observation of a Discrete Time Crystal, Nature (London) 543, 217 (2017).

[16] S. Choi, J. Choi, R. Landig, G. Kucsko, H. Zhou, J. Isoya, F. Jelezko, S. Onoda, H. Sumiya, V. Khemani, C. von Keyserlingk, N. Y. Yao, E. Demler, and M. D. Lukin, Observation of Discrete Time-Crystalline Order in a
Disordered Dipolar Many-Body System, Nature (London) 543, 221 (2017).

[17] J. Rovny, R. L. Blum, and S. E. Barrett, Observation of Discrete-Time-Crystal Signatures in an Ordered Dipolar Many-Body System, Phys. Rev. Lett. 120, 180603 (2018).

[18] I. Bloch, J. Dalibard, and W. Zwerger, Many-Body Physics with Ultracold Gases, Rev. Mod. Phys. 80, 885 (2008).

[19] D. Leibfried, R. Blatt, C. Monroe, and D. Wineland, Quantum Dynamics of Single Trapped Ions, Rev. Mod. Phys. 75, 281 (2003).

[20] L.-M. Duan and C. Monroe, Colloquium: Quantum Networks with Trapped Ions, Rev. Mod. Phys. 82, 1209 (2010).

[21] R. Blatt and C.F. Roos, Quantum Simulations with Trapped Ions, Nat. Phys. 8, 277 (2012).

[22] M. W. Doherty, N. B. Manson, P. Delaney, F. Jelezko, J. Wrachtrup, and L.C.L. Hollenberg, The NitrogenVacancy Colour Centre in Diamond, Phys. Rep. 528, 1 (2013).

[23] R. Schirhagl, K. Chang, M. Loretz, and C. L. Degen, Nitrogen-Vacancy Centers in Diamond: Nanoscale Sensors for Physics and Biology, Annu. Rev. Phys. Chem. 65, 83 (2014).

[24] J. Kelly et al., State Preservation by Repetitive Error Detection in a Superconducting Quantum Circuit, Nature (London) 519, 66 (2015).

[25] P. Roushan et al., Spectroscopic Signatures of Localization with Interacting Photons in Superconducting Qubits, Science 358, 1175 (2017).

[26] J. M. Deutsch, Quantum Statistical Mechanics in a Closed System, Phys. Rev. A 43, 2046 (1991).

[27] M. Srednicki, Chaos and Quantum Thermalization, Phys. Rev. E 50, 888 (1994).

[28] L. D'Alessio and M. Rigol, Long-Time Behavior of Isolated Periodically Driven Interacting Lattice Systems, Phys. Rev. X 4, 041048 (2014).

[29] A. Lazarides, A. Das, and R. Moessner, Equilibrium States of Generic Quantum Systems Subject to Periodic Driving, Phys. Rev. E 90, 012110 (2014).

[30] P. Ponte, A. Chandran, Z. Papić, and D. A. Abanin, Periodically Driven Ergodic and Many-Body Localized Quantum Systems, Ann. Phys. (Amsterdam) 353, 196 (2015).

[31] D. A. Abanin, W. De Roeck, and F. Huveneers, Exponentially Slow Heating in Periodically Driven Many-Body Systems, Phys. Rev. Lett. 115, 256803 (2015).

[32] T. Kuwahara, T. Mori, and K. Saito, Floquet-Magnus Theory and Generic Transient Dynamics in Periodically Driven Many-Body Quantum Systems, Ann. Phys. (Amsterdam) 367, 96 (2016).

[33] T. Mori, T. Kuwahara, and K. Saito, Rigorous Bound on Energy Absorption and Generic Relaxation in Periodically Driven Quantum Systems, Phys. Rev. Lett. 116, 120401 (2016).

[34] D. Abanin, W. De Roeck, W. W. Ho, and F. Huveneers, A Rigorous Theory of Many-Body Prethermalization for Periodically Driven and Closed Quantum Systems, Commun. Math. Phys. 354, 809 (2017).

[35] D. A. Abanin, W. De Roeck, W. W. Ho, and F. Huveneers, Effective Hamiltonians, Prethermalization, and Slow Energy Absorption in Periodically Driven Many-Body Systems, Phys. Rev. B 95, 014112 (2017). 
[36] S. A. Weidinger and M. Knap, Floquet Prethermalization and Regimes of Heating in a Periodically Driven, Interacting Quantum System, Sci. Rep. 7, 45382 (2017).

[37] A. Lazarides, A. Das, and R. Moessner, Fate of ManyBody Localization under Periodic Driving, Phys. Rev. Lett. 115, 030402 (2015).

[38] D. A. Abanin, W. De Roeck, and F. Huveneers, Theory of Many-Body Localization in Periodically Driven Systems, Ann. Phys. (Amsterdam) 372, 1 (2016).

[39] T.-S. Ho, S.-I. Chu, and J. V. Tietz, Semiclassical ManyMode Floquet Theory, Chem. Phys. Lett. 96, 464 (1983).

[40] J. M. Luck, H. Orland, and U. Smilansky, On the Response of a Two-Level Quantum System to a Class of TimeDependent Quasiperiodic Perturbations, J. Stat. Phys. 53, 551 (1988).

[41] H. R. Jauslin and J. L. Lebowitz, Spectral and Stability Aspects of Quantum Chaos, Chaos 1, 114 (1991).

[42] H. R. Jauslin and J. L. Lebowitz, Generalized Floquet Operator for Quasiperiodically Driven Quantum Systems, in Mathematical Physics $X$, edited by K. Schmüdgen (Springer, Berlin, Heidelberg, 1992), pp. 313-316.

[43] P. M. Blekher, H. R. Jauslin, and J. L. Lebowitz, Floquet Spectrum for Two-Level Systems in Quasiperiodic TimeDependent Fields, J. Stat. Phys. 68, 271 (1992).

[44] U. Feudel, A. S. Pikovsky, and M. A. Zaks, Correlation Properties of a Quasiperiodically Forced Two-Level System, Phys. Rev. E 51, 1762 (1995).

[45] G. Casati, I. Guarneri, and D. L. Shepelyansky, Anderson Transition in a One-Dimensional System with Three Incommensurate Frequencies, Phys. Rev. Lett. 62, 345 (1989).

[46] À. Jorba and C. Simó, On the Reducibility of Linear Differential Equations with Quasiperiodic Coefficients, J. Differential Equations 98, 111 (1992).

[47] D. Bambusi and S. Graffi, Time Quasi-periodic Unbounded Perturbations of Schrödinger Operators and KAM Methods, Commun. Math. Phys. 219, 465 (2001).

[48] G. Gentile, Quasi-periodic Solutions for Two-Level Systems, Commun. Math. Phys. 242, 221 (2003).

[49] S.-I. Chu and D. A. Telnov, Beyond the Floquet Theorem: Generalized Floquet Formalisms and Quasienergy Methods for Atomic and Molecular Multiphoton Processes in Intense Laser Fields, Phys. Rep. 390, 1 (2004).

[50] R. Gommers, S. Denisov, and F. Renzoni, Quasiperiodically Driven Ratchets for Cold Atoms, Phys. Rev. Lett. 96, 240604 (2006).

[51] J. Chabé, G. Lemarié, B. Grémaud, D. Delande, P. Szriftgiser, and J.C. Garreau, Experimental Observation of the Anderson Metal-Insulator Transition with Atomic Matter Waves, Phys. Rev. Lett. 101, 255702 (2008).

[52] G. Lemarié, H. Lignier, D. Delande, P. Szriftgiser, and J. C. Garreau, Critical State of the Anderson Transition: Between a Metal and an Insulator, Phys. Rev. Lett. 105, 090601 (2010).

[53] D. Cubero and F. Renzoni, Control of Transport in Two-Dimensional Systems via Dynamical Decoupling of Degrees of Freedom with Quasiperiodic Driving Fields, Phys. Rev. E 86, 056201 (2012).

[54] A. Verdeny, J. Puig, and F. Mintert, Quasi-periodically Driven Quantum Systems, Z. Nat. A 71, 897 (2016).
[55] S. Nandy, A. Sen, and D. Sen, Aperiodically Driven Integrable Systems and Their Emergent Steady States, Phys. Rev. X 7, 031034 (2017).

[56] D. Cubero and F. Renzoni, Asymptotic Theory of Quasiperiodically Driven Quantum Systems, Phys. Rev. E 97, 062139 (2018).

[57] S. Nandy, A. Sen, and D. Sen, Steady States of a Quasiperiodically Driven Integrable System, Phys. Rev. B 98, 245144 (2018).

[58] S. Ray, S. Sinha, and D. Sen, Dynamics of Quasiperiodically Driven Spin Systems, Phys. Rev. E 100, 052129 (2019).

[59] I. Martin, G. Refael, and B. Halperin, Topological Frequency Conversion in Strongly Driven Quantum Systems, Phys. Rev. X 7, 041008 (2017).

[60] Y. Peng and G. Refael, Time-Quasiperiodic Topological Superconductors with Majorana Multiplexing, Phys. Rev. B 98, 220509(R) (2018).

[61] P. J. D. Crowley, I. Martin, and A. Chandran, Topological Classification of Quasiperiodically Driven Quantum Systems, Phys. Rev. B 99, 064306 (2019).

[62] F. Nathan, I. Martin, and G. Refael, Topological Frequency Conversion in a Driven Dissipative Quantum Cavity, Phys. Rev. B 99, 094311 (2019).

[63] P. J. D. Crowley, I. Martin, and A. Chandran, Half-Integer Quantized Response in Strongly Driven Quantum Systems, arXiv:1908.08062.

[64] P. T. Dumitrescu, R. Vasseur, and A. C. Potter, Logarithmically Slow Relaxation in Quasiperiodically Driven Random Spin Chains, Phys. Rev. Lett. 120, 070602 (2018).

[65] K. Giergiel, A. Kuroś, and K. Sacha, Discrete Time Quasicrystals, Phys. Rev. B 99, 220303(R) (2019).

[66] H. Zhao, F. Mintert, and J. Knolle, Floquet Time Spirals and Stable Discrete-Time Quasicrystals in Quasiperiodically Driven Quantum Many-Body Systems, Phys. Rev. B 100, 134302 (2019).

[67] S. Autti, V. B. Eltsov, and G. E. Volovik,Observation of a Time Quasicrystal and Its Transition to a Superfluid Time Crystal, Phys. Rev. Lett. 120, 215301 (2018).

[68] A. Pizzi, J. Knolle, and A. Nunnenkamp, Period-n Discrete Time Crystals and Quasicrystals with Ultracold Bosons, Phys. Rev. Lett. 123, 150601 (2019).

[69] D. V. Else and C. Nayak, Classification of Topological Phases in Periodically Driven Interacting Systems, Phys. Rev. B 93, 201103(R) (2016).

[70] N. H. Lindner, E. Berg, and M. S. Rudner, Universal Chiral Quasisteady States in Periodically Driven ManyBody Systems, Phys. Rev. X 7, 011018 (2017).

[71] A. Haldar, R. Moessner, and A. Das, Onset of Floquet Thermalization, Phys. Rev. B 97, 245122 (2018).

[72] A. Haldar, D. Sen, R. Moessner, and A. Das, Scars in Strongly Driven Floquet Matter: Resonance vs Emergent Conservation Laws, arXiv:1909.04064.

[73] M. Bukov, L. D’Alessio, and A. Polkovnikov, Universal High-Frequency Behavior of Periodically Driven Systems: From Dynamical Stabilization to Floquet Engineering, Adv. Phys. 64, 139 (2015).

[74] R. Nandkishore and D. A. Huse, Many-Body Localization and Thermalization in Quantum Statistical Mechanics, Annu. Rev. Condens. Matter Phys. 6, 15 (2015). 
[75] F. Alet and N. Laflorencie, Many-Body Localization: An Introduction and Selected Topics, Comp. Rend. Phys. 19, 498 (2018).

[76] S. A. Parameswaran and R. Vasseur, Many-Body Localization, Symmetry and Topology, Rep. Prog. Phys. 81, 082501 (2018).

[77] D. A. Abanin, E. Altman, I. Bloch, and M. Serbyn, Colloquium: Many-Body Localization, Thermalization, and Entanglement, Rev. Mod. Phys. 91, 021001 (2019).

[78] T. Kitagawa, T. Oka, A. Brataas, L. Fu, and E. Demler, Transport Properties of Nonequilibrium Systems under the Application of Light: Photoinduced Quantum Hall Insulators without Landau Levels, Phys. Rev. B 84, 235108 (2011).

[79] A. G. Grushin, À. Gómez-León, and T. Neupert, Floquet Fractional Chern Insulators, Phys. Rev. Lett. 112, 156801 (2014).

[80] F. Meinert, M. J. Mark, K. Lauber, A. J. Daley, and H.-C. Nägerl, Floquet Engineering of Correlated Tunneling in the Bose-Hubbard Model with Ultracold Atoms, Phys. Rev. Lett. 116, 205301 (2016).

[81] N. R. Cooper, J. Dalibard, and I. B. Spielman, Topological Bands for Ultracold Atoms, Rev. Mod. Phys. 91, 015005 (2019).

[82] C. H. Lee, W. W. Ho, B. Yang, J. Gong, and Z. Papić, Floquet Mechanism for Non-Abelian Fractional Quantum Hall States, Phys. Rev. Lett. 121, 237401 (2018).

[83] A. Clark, Elements of Abstract Algebra (Dover Publications, New York, 1984).

[84] D. Levine and P. J. Steinhardt, Quasicrystals. I. Definition and Structure, Phys. Rev. B 34, 596 (1986).

[85] J. E. S. Socolar and P. J. Steinhardt, Quasicrystals. II. UnitCell Configurations, Phys. Rev. B 34, 617 (1986).

[86] C. W. von Keyserlingk, V. Khemani, and S. L. Sondhi, Absolute Stability and Spatiotemporal Long-Range Order in Floquet Systems, Phys. Rev. B 94, 085112 (2016).

[87] I.-D. Potirniche, A. C. Potter, M. Schleier-Smith, A. Vishwanath, and N. Y. Yao, Floquet Symmetry-Protected Topological Phases in Cold-Atom Systems, Phys. Rev. Lett. 119, 123601 (2017).

[88] D. A. Huse, R. Nandkishore, V. Oganesyan, A. Pal, and S. L. Sondhi, Localization-Protected Quantum Order, Phys. Rev. B 88, 014206 (2013).

[89] R. Vosk and E. Altman, Dynamical Quantum Phase Transitions in Random Spin Chains, Phys. Rev. Lett. 112, 217204 (2014).

[90] D. Pekker, G. Refael, E. Altman, E. Demler, and V. Oganesyan, Hilbert-Glass Transition: New Universality of Temperature-Tuned Many-Body Dynamical Quantum Criticality, Phys. Rev. X 4, 011052 (2014).

[91] J. A. Kjäll, J. H. Bardarson, and F. Pollmann, Many-Body Localization in a Disordered Quantum Ising Chain, Phys. Rev. Lett. 113, 107204 (2014).

[92] M. Serbyn, Z. Papić, and D. A. Abanin, Quantum Quenches in the Many-Body Localized Phase, Phys. Rev. B 90, 174302 (2014).

[93] We thank the anonymous referee for pointing this out to us.

[94] W. De Roeck and F. Huveneers, Stability and Instability Towards Delocalization in Many-Body Localization Systems, Phys. Rev. B 95, 155129 (2017).
[95] D. J. Luitz, F. Huveneers, and W. De Roeck, How a Small Quantum Bath Can Thermalize Long Localized Chains, Phys. Rev. Lett. 119, 150602 (2017).

[96] W. De Roeck and J. Z. Imbrie, Many-Body Localization: Stability and Instability, Phil. Trans. R. Soc. A 375, 20160422 (2017).

[97] S. Gopalakrishnan and D. A. Huse, Instability of ManyBody Localized Systems as a Phase Transition in a Nonstandard Thermodynamic Limit, Phys. Rev. B 99, 134305 (2019).

[98] P. Bordia, H. Lüschen, S. Scherg, S. Gopalakrishnan, M. Knap, U. Schneider, and I. Bloch, Probing Slow Relaxation and Many-Body Localization in Two-Dimensional Quasiperiodic Systems, Phys. Rev. X 7, 041047 (2017).

[99] J.-y. Choi, S. Hild, J. Zeiher, P. Schauss, A. Rubio-Abadal, T. Yefsah, V. Khemani, D. A. Huse, I. Bloch, and C. Gross, Exploring the Many-Body Localization Transition in Two Dimensions, Science 352, 1547 (2016).

[100] B. Bauer and C. Nayak, Area Laws in a Many-Body Localized State and Its Implications for Topological Order, J. Stat. Mech. (2013) P09005.

[101] Y. Bahri, R. Vosk, E. Altman, and A. Vishwanath, Localization and Topology Protected Quantum Coherence at the Edge of Hot Matter, Nat. Commun. 6, 7341 (2015).

[102] A. Chandran, V. Khemani, C. R. Laumann, and S. L. Sondhi, Many-Body Localization and Symmetry-Protected Topological Order, Phys. Rev. B 89, 144201 (2014).

[103] C. W. von Keyserlingk and S. L. Sondhi, Phase Structure of One-Dimensional Interacting Floquet Systems. I. Abelian Symmetry-Protected Topological Phases, Phys. Rev. B 93, 245145 (2016).

[104] A. C. Potter, T. Morimoto, and A. Vishwanath, Classification of Interacting Topological Floquet Phases in One Dimension, Phys. Rev. X 6, 041001 (2016).

[105] R. Roy and F. Harper, Abelian Floquet SymmetryProtected Topological Phases in One Dimension, Phys. Rev. B 94, 125105 (2016).

[106] R. Roy and F. Harper, Floquet Topological Phases with Symmetry in All Dimensions, Phys. Rev. B 95, 195128 (2017).

[107] D. V. Else, Time Crystals and Space Crystals: Strongly Correlated Phases of Matter with Space-Time Symmetries, Ph.D. thesis, University of California, Santa Barbara, 2018.

[108] V. Turaev, Homotopy Field Theory in Dimension 2 and Group-Algebras, arXiv:math.QA/9910010.

[109] V. Turaev, Homotopy Field Theory in Dimension 3 and Crossed Group-Categories, arXiv:math.GT/0005291.

[110] A. Kitaev, Anyons in an Exactly Solved Model and Beyond, Ann. Phys. (Amsterdam) 321, 2 (2006).

[111] J. Lurie, On the Classification of Topological Field Theories, arXiv:0905.0465.

[112] A. Kitaev, Institute for Pure Applied Mathematics, UCLA, 2015, http://www.ipam.ucla.edu/abstract/?tid=12389\& pcode $=$ STQ2015.

[113] R. Thorngren and D. V. Else, Gauging Spatial Symmetries and the Classification of Topological Crystalline Phases, Phys. Rev. X 8, 011040 (2018).

[114] C. Z. Xiong, Minimalist Approach to the Classification of Symmetry Protected Topological Phases, J. Phys. A 51, 445001 (2018). 
[115] D. V. Else and R. Thorngren, Crystalline Topological Phases as Defect Networks, Phys. Rev. B 99, 115116 (2019).

[116] R. Dijkgraaf and E. Witten, Topological Gauge Theories and Group Cohomology, Commun. Math. Phys. 129, 393 (1990).

[117] X. Chen, Z.-C. Gu, and X.-G. Wen, Classification of Gapped Symmetric Phases in One-Dimensional Spin Systems, Phys. Rev. B 83, 035107 (2011).

[118] N. Schuch, D. Pérez-García, and I. Cirac, Classifying Quantum Phases Using Matrix Product States and Projected Entangled Pair States, Phys. Rev. B 84, 165139 (2011).

[119] X. Chen, Z.-C. Gu, Z.-X. Liu, and X.-G. Wen, Symmetry Protected Topological Orders and the Group Cohomology of Their Symmetry Group, Phys. Rev. B 87, 155114 (2013).

[120] X.-G. Wen, Symmetry-Protected Topological Invariants of Symmetry-Protected Topological Phases of Interacting Bosons and Fermions, Phys. Rev. B 89, 035147 (2014).

[121] A.C. Potter and T. Morimoto, Dynamically Enriched Topological Orders in Driven Two-Dimensional Systems, Phys. Rev. B 95, 155126 (2017).

[122] C. L. Kane and E. J. Mele, $Z_{2}$ Topological Order and the Quantum Spin Hall Effect, Phys. Rev. Lett. 95, 146802 (2005).

[123] F. Pollmann, E. Berg, A. M. Turner, and M. Oshikawa, Symmetry Protection of Topological Phases in OneDimensional Quantum Spin Systems, Phys. Rev. B 85, 075125 (2012).

[124] M. Levin and Z.-C. Gu, Braiding Statistics Approach to Symmetry-Protected Topological Phases, Phys. Rev. B 86, 115109 (2012).

[125] M. Barkeshli, P. Bonderson, M. Cheng, and Z. Wang, Symmetry Fractionalization, Defects, and Gauging of Topological Phases, Phys. Rev. B 100, 115147 (2019).

[126] X. Chen, Y.-M. Lu, and A. Vishwanath, SymmetryProtected Topological Phases from Decorated Domain Walls, Nat. Commun. 5, 3507 (2014).

[127] W. M. Schmidt, Norm Form Equations, Ann. Math. 96, 526 (1972).

[128] W. M. Schmidt, Diophantine Approximation (SpringerVerlag, Berlin, 1980).

[129] M. C. Tran, A. Ehrenberg, A. Y. Guo, P. Titum, D. A. Abanin, and A. V. Gorshkov, Locality and Heating in Periodically Driven, Power-Law-Interacting Systems, Phys. Rev. A 100, 052103 (2019).

[130] F. Machado, D. V. Else, G. D. Kahanamoku-Meyer, C. Nayak, and N. Y. Yao, Prethermal Phases of Nonequilibrium Matter in Long-Range Interacting Systems, Phys. Rev. X 10, 011043 (2020).

[131] W. W. Ho, I. Protopopov, and D. A. Abanin, Bounds on Energy Absorption and Prethermalization in Quantum Systems with Long-Range Interactions, Phys. Rev. Lett. 120, 200601 (2018).

[132] D. V. Else, P. Fendley, J. Kemp, and C. Nayak, Prethermal Strong Zero Modes and Topological Qubits, Phys. Rev. X 7, 041062 (2017).

[133] E. van Nieuwenburg, Y. Baum, and G. Refael, From Bloch Oscillations to Many-Body Localization in Clean
Interacting Systems, Proc. Natl. Acad. Sci. USA 116, 9269 (2019).

[134] M. Schulz, C. A. Hooley, R. Moessner, and F. Pollmann, Stark Many-Body Localization, Phys. Rev. Lett. 122, 040606 (2019).

[135] V. Khemani, M. Hermele, and R. M. Nandkishore, Localization from Shattering: Higher Dimensions and Physical Realizations, arXiv:1910.01137.

[136] P. Sala, T. Rakovszky, R. Verresen, M. Knap, and F. Pollmann, Ergodicity-Breaking Arising from Hilbert Space Fragmentation in Dipole-Conserving Hamiltonians, Phys. Rev. X 10, 011047 (2020).

[137] V. Khemani and R. Nandkishore, Local Constraints Can Globally Shatter Hilbert Space: A New Route to Quantum Information Protection, arXiv:1904.04815.

[138] T. Rakovszky, P. Sala, R. Verresen, M. Knap, and F. Pollmann, Statistical Localization: From Strong Fragmentation to Strong Edge Modes, Phys. Rev. B 101, 125126 (2020).

[139] H. C. Po, L. Fidkowski, T. Morimoto, A. C. Potter, and A. Vishwanath, Chiral Floquet Phases of Many-Body Localized Bosons, Phys. Rev. X 6, 041070 (2016).

[140] F. Harper and R. Roy, Floquet Topological Order in Interacting Systems of Bosons and Fermions, Phys. Rev. Lett. 118, 115301 (2017).

[141] H. C. Po, L. Fidkowski, A. Vishwanath, and A. C. Potter, Radical Chiral Floquet Phases in a Periodically Driven Kitaev Model and Beyond, Phys. Rev. B 96, 245116 (2017).

[142] L. Fidkowski, H. C. Po, A. C. Potter, and A. Vishwanath, Interacting Invariants for Floquet Phases of Fermions in Two Dimensions, Phys. Rev. B 99, 085115 (2019).

[143] G. Kucsko, S. Choi, J. Choi, P. C. Maurer, H. Zhou, R. Landig, H. Sumiya, S. Onoda, J. Isoya, F. Jelezko, E. Demler, N. Y. Yao, and M. D. Lukin, Critical Thermalization of a Disordered Dipolar Spin System in Diamond, Phys. Rev. Lett. 121, 023601 (2018).

[144] J. Choi, H. Zhou, S. Choi, R. Landig, W. W. Ho, J. Isoya, F. Jelezko, S. Onoda, H. Sumiya, D. A. Abanin, and M. D. Lukin, Probing Quantum Thermalization of a Disordered Dipolar Spin Ensemble with Discrete Time-Crystalline Order, Phys. Rev. Lett. 122, 043603 (2019).

[145] A. Kapustin, Symmetry Protected Topological Phases, Anomalies, and Cobordisms: Beyond Group Cohomology, arXiv:1403.1467.

[146] A. Kapustin, R. Thorngren, A. Turzillo, and Z. Wang, Fermionic Symmetry Protected Topological Phases and Cobordisms, J. High Energy Phys. 12 (2015) 1.

[147] D. S. Freed and M. J. Hopkins, Reflection Positivity and Invertible Topological Phases," arXiv:1604.06527.

[148] A. Kirillov, Jr., On g-Equivariant Modular Categories, arXiv:math/0401119.

[149] P. Etingof, D. Nikshych, V. Ostrik, and with an appendix by E. Meir, Fusion Categories and Homotopy Theory, arXiv:0909.3140.

[150] A. Hatcher, Algebraic Topology (Cambridge University Press, New York, 2001).

[151] Y. Bugeaud, Distribution Modulo One and Diophantine Approximation (Cambridge University Press, New York, 2012). 